\title{
Differential dynamics of peripheral immune responses to acute SARS-CoV-2 infection in older adults
}

\author{
Sloan A. Lewis ${ }^{1,2,9}$, Suhas Sureshchandra1,2,9, Michael Z. Zulu ${ }^{101,2}$, Brianna Doratt ${ }^{1,2}$, Allen Jankeel', \\ Izabela Coimbra Ibraim ${ }^{1}$, Amanda N. Pinski ${ }^{1}$, Nicholas S. Rhoades ${ }^{1,2}$, Micaila Curtis $\mathbb{1}^{1}$, Xiwen Jiang ${ }^{3}$, \\ Delia Tifrea ${ }^{4}$, Frank Zaldivar ${ }^{5}$, Weining Shen $\mathbb{1}^{3}{ }^{3}$, Robert A. Edwards ${ }^{4}$, Daniel Chow ${ }^{6}$, Dan Cooper ${ }^{2,7}$, \\ Alpesh Amin ${ }^{7}$ and Ilhem Messaoudi $1,2,8 凶$
}

In this study, peripheral blood mononuclear cells from young and old patients with COVID-19 were examined phenotypically, transcriptionally and functionally to reveal age-, time- and severity-specific adaptations. Gene signatures within memory B cells and plasmablasts correlated with reduced frequency of antigen-specific B cells and neutralizing antibodies in older patients with severe COVID-19. Moreover, these patients exhibited exacerbated T cell lymphopenia, which correlated with lower plasma interleukin-2, and diminished antigen-specific $T$ cell responses. Single-cell RNA sequencing revealed augmented signatures of activation, exhaustion, cytotoxicity and type I interferon signaling in memory $\mathbf{T}$ and natural killer cells with age. Although cytokine storm was evident in both age groups, older individuals exhibited elevated levels of myeloid cell recruiting factors. Furthermore, we observed redistribution of monocyte and dendritic cell subsets and emergence of a suppressive phenotype with severe disease, which was reversed only in young patients over time. This analysis provides new insights into the impact of aging on COVID-19.

T he coronavirus disease 2019 (COVID-19) pandemic, caused by the severe acute respiratory syndrome coronavirus 2 (SARS-CoV-2), has resulted in over 40 million cases and 700,000 deaths in the US alone. SARS-CoV-2 targets lung airway and alveolar epithelial cells, vascular endothelial cells and macrophages in the lung. Infection triggers a vigorous innate and adaptive immune response that culminates in viral clearance or, in the case of severe infection, the development of a cytokine storm, acute respiratory distress syndrome (ARDS) and multi-organ failure ${ }^{1}$. The estimated mortality rate of COVID-19 in the US is $1-2 \%$, while approximately $80 \%$ of patients are thought to be asymptomatic or experience mild disease symptoms $\mathrm{s}^{2,3}$.

Although there are many questions that remain to be answered regarding the large discrepancies in disease outcomes, it is clear that advanced age is a leading risk factor for severe COVID-19 and death $\mathrm{h}^{4-6}$. Indeed, the case fatality rate worldwide increases progressively with age $(0.4 \%$ at age $55,1.4 \%$ at age $65,4.6 \%$ at age 75 and $15 \%$ at age 85$)^{7,8}$. The highest proportion of patients who develop severe respiratory complications, such as respiratory failure, dyspnea, pneumonia, ARDS and death are older adults ${ }^{9-14}$. Age-related changes in immunity, dubbed 'immunosenescence', result in a marked increase in susceptibility to respiratory viral infections and attenuated vaccine responses ${ }^{15,16}$. Some of the hallmarks of immunosenescence include decreased frequency of naive $\mathrm{T}$ and $\mathrm{B}$ cells, increased prevalence of effector/terminally differentiated memory cells, as well as increased levels of circulating inflammatory mediators including interleukin-6 (IL-6) and C-reactive protein referred to as 'inflammaging' ${ }^{17}$. Despite the rich literature on COVID-19 pathogenesis, the specific immune mechanisms associated with the increased severity of COVID-19 among older adults and the immune correlates of mild and severe disease in the older population remain elusive.

While age-associated immune changes in the lung undoubtedly contribute largely to a reduced ability to fight SARS-CoV-2 infec$\operatorname{tion}^{18}$, such studies are challenging to conduct. Consequently, most studies to date have relied on profiling circulating immune cells ${ }^{19}$. These studies have shown that severe COVID-19 is mediated by dysregulated innate and adaptive immune responses. Elevated levels of IL-1, IL-6, IL-8 and CXCL10 in the blood have been associated with more severe infection or death ${ }^{20-24}$. Disruptions in distribution, activation as well as gene expression of circulating immune cells have been identified by single-cell RNA sequencing (scRNA-seq). Notably, a pronounced decrease of human leukocyte antigen - DR isotype (HLA-DR) on monocytes ${ }^{22,25,26}$, an increase in nonclassical $\mathrm{CD}^{2} 6^{+}$monocytes $^{22}$ and a decrease in the frequency of total dendritic cells (DCs) ${ }^{26}$ compared to patients with moderate or mild COVID-19 and healthy controls. Additionally, mild disease results in increased interferon (IFN) signaling gene expression, whereas severe disease leads to impaired type I IFN responses ${ }^{27}$ and reduced ability to respond to a secondary stimulation in monocytes ${ }^{28}$.

'Department of Molecular Biology and Biochemistry, University of California, Irvine, Irvine, CA, USA. ${ }^{2}$ Institute for Immunology, University of California, Irvine, Irvine, CA, USA. ${ }^{3}$ Department of Statistics, University of California, Irvine, Irvine, CA, USA. ${ }^{4}$ Department of Pathology and Laboratory Medicine,

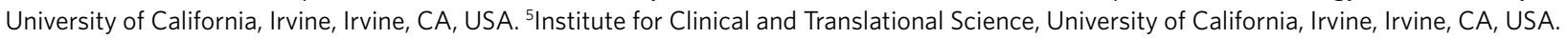
${ }^{6}$ Department of Radiology, University of California, Irvine, Irvine, CA, USA. ${ }^{7}$ Department of Medicine, University of California, Irvine, Irvine, CA, USA. ${ }^{8}$ Center for Virus Research, University of California, Irvine, Irvine, CA, USA. ${ }^{9}$ These authors contributed equally: Sloan A. Lewis, Suhas Sureshchandra. 凶e-mail: imessaou@uci.edu 
Studies examining natural killer (NK) cells have reported reduced numbers of total NK cells with disease severity but no differences within subsets ${ }^{29-31}$. Similarly, NK cells have been shown to upregulate IFN response genes but have an exhausted phenotype and reduced cytolytic activity ${ }^{29,30}$. Another hallmark of severe COVID19 is lymphopenia ${ }^{32}$ that is accompanied by increased $\mathrm{T}$ cell activation and exhaustion ${ }^{30,33-36}$.

Data from these studies have begun to paint a picture of significant immune dysregulation with COVID-19 that is potentially exacerbated in older patients ${ }^{37,38}$. Regardless, gaps in our knowledge remain. Notably, comprehensive longitudinal studies that include both young and older individuals with varying disease severity (from mild to fatal) as well age-matched controls and integrated transcriptional and functional analyses are needed. In this study, we carried out a longitudinal phenotypic, transcriptional and functional analysis of peripheral blood mononuclear cells (PBMCs) from young and old patients with COVID-19 compared to agematched healthy controls. Analysis of circulating immune mediators revealed higher levels of cytokines important for $\mathrm{T}$ cell survival and IFN $\beta$ in young individuals, while older individuals exhibited higher levels of myeloid cell recruiting chemokines. scRNA-seq analysis revealed dampened activation signatures in plasmablasts and memory B cells isolated from older individuals that correlated with reduced neutralizing titers. Analysis of the T cell subsets revealed heightened activation and exhaustion states with severe disease and an age-associated exacerbation of IFN and cytotoxicity signaling. Functionally, this led to immune paralysis in older individuals, with worsened $\mathrm{T}$ cell lymphopenia and diminished antiSARS-CoV-2 T cell responses. Similarly, signatures of increased exhaustion and cytotoxicity markers were more evident in NK cells from older individuals. Finally, severe COVID-19 resulted in a functional rewiring of monocytes and DCs toward a more regulatory phenotype where nuclear factor kappa-light-chain-enhancer of activated B cell $(\mathrm{NF}-\kappa \mathrm{B})$-mediated responses were diminished while type I IFN responses were enhanced. The findings presented enhance our understanding of age-mediated disruptions in antiviral responses and will aid the design of therapeutics and vaccines for this at-risk population.

\section{Results}

Magnitude of cytokine storm in severe COVID-19 is modulated by age. To comprehensively assess the peripheral immune response to SARS-CoV-2 infection with age, we performed a combination of immunological, single-cell transcriptomic and functional assays (Fig. 1a) using blood samples obtained from 49 healthy donors ( 37 young and $12 \mathrm{old}), 25 \mathrm{mild} /$ asymptomatic COVID-19 patients (13 young and 12 old) and 51 patients severely infected with COVID19 (35 young and 16 old) with longitudinal sampling based on days post-symptom (DPS) onset (median approximately 10 DPS). Each group contained young ( $<60$ years, median age 42.5 years) and old patients ( $\geq 60$ years, median age 69) including 6 patients ( 2 young and 4 old) who succumbed to infection (Supplementary Table 1).

We first began by measuring circulating levels of key immune mediators using Luminex with a focus on factors significantly altered with severity and age. While several analytes were elevated with severe disease (Extended Data Fig. 1a), levels of IP-10, granulocyte-macrophage colony-stimulating factor (GM-CSF), IL-10 and TNF-related apoptosis-inducing ligand (TRAIL) were more elevated in older individuals with severe disease compared to young individuals. On the other hand, granzyme B and regulated on activation, normal T cell expressed and secreted (RANTES) were markedly lower in older individuals with severe disease relative to their young counterparts (Extended Data Fig. 1a).

We next tracked temporal changes in plasma cytokines, chemokines and growth factors in young patients, old patients who survived and old patients who succumbed to COVID-19. Linear regression analysis revealed significant increase in T cell- (IL-2, IL-7, tumor necrosis factor- $\alpha$ (TNF), macrophage inflammatory protein-1 $\beta$ (MIP-1 $\beta$ ), IL-4, IL-5, IL-13, CD40L) and myeloid cellassociated factors (IL- $1 \alpha$, IL-1 $\beta$, GRO- $\beta$, CX3CL1) and growth factors (platelet-derived growth factor-AA (PDGF-AA), transforming growth factor- $\alpha$ (TGF $\alpha$ ), epidermal growth factor (EGF)) exclusively in young individuals with severe disease (Extended Data Fig. 1b). To test differences with age, we used an analysis of covariance (ANCOVA) on individual factors treated independently with DPS as a continuous covariate. Our analysis revealed sharp increase in circulating $\mathrm{T}_{\mathrm{H}} 2$ cytokine IL- 5 and type I IFN $\beta$ in young individuals relative to older individuals with severe disease (Fig. 1b). On the other hand, levels of IFN $\alpha$ were reduced significantly in young and older individuals who survived over time (Fig. 1b). Interestingly, within the older group, a number of myeloid cell-associated growth factors (IL-3, granulocyte colony-stimulating factor (G-CSF), (GM-CSF)), chemokines (MIP-3 $\beta$, monocyte chemoattractant protein 1 (MCP1 ), eotaxin) and regulatory cytokines (IL-1RA and IL-10) were significantly different between older individuals who survived versus those who succumbed to infection (Fig. 1b). Furthermore, levels of IFN $\gamma$ were significantly positively correlated while levels of GM-CSF were significantly negatively correlated with DPS only in older patients who succumbed to infection (Fig. 1b).

Finally, we used supervised random forest modeling to identify factors that could have early (1-5 DPS) predictive value of COVID19 outcomes (Extended Data Fig. 1c,d). Independent of age, plasma levels of IL-17A, IL-33, IP-10, IL-15, MIP-1 $\beta$ and IFN $\alpha$ were the strongest predictors of disease severity (Extended Data Fig. 1c,d). IL-17 levels were highly predictive of mild disease regardless of age, while MIP-1 $\beta$ levels were more predictive of severe disease in older adults (Extended Data Fig. 1d).

Peripheral immune adaptations with disease severity and age. To assess the impact of age and COVID-19 disease severity on peripheral immune adaptations, we profiled the PBMCs from each patient by flow cytometry. Analysis of major populations in the blood including B cells, T cells, NK cells, DCs and monocytes identified significant differences in immune cell frequencies, particularly with disease severity and age (Fig. 1c). Specifically, decreased percentages of naive $B$ cells and a concomitant increase in memory B cell fractions was observed in older patients with severe disease compared to healthy donors. Furthermore, severe disease in older patients was associated with significant decrease in central memory CD4 $\left(\mathrm{T}_{\mathrm{CM}}\right)$ and naive $\mathrm{CD} 8$ cells relative to their young counterparts (Fig. 1c). In contrast, mild disease was associated with an expansion of $\mathrm{CD} 8 \mathrm{~T}_{\mathrm{CM}}$ cells in both young and older patients, albeit to a greater extent in older individuals. The frequencies of DC subsets decreased significantly in both old and young patients regardless of disease severity, whereas monocytosis was observed only in young patients with severe COVID-19 (Fig. 1c).

To further characterize the unique aspects of the peripheral immune response to acute COVID-19 with age, PBMCs from 16 patients (young mild $=4$; young severe $=4$; old mild $=4$; old severe $=4$ ) and 8 healthy donors (young $=4$; old $=4$ ) were profiled by scRNA-seq (Fig. 1a). For patients with severe disease, we profiled PBMCs at three time points (ranging from DPS 2 to DPS 22) over the course of acute infection (Extended Data Fig. 2a). An average of 2,082 cells were sequenced per patient and time point for a total of 92,648 cells (Extended Data Fig. 2b). Principal component analysis and dimensional reduction using uniform manifold approximation and projection (UMAP) revealed distinct clustering into the major peripheral innate and adaptive immune subsets (Extended Data Fig. 2c). Clusters were annotated based on highly expressed marker genes using Seurat's FindMarkers function (Extended Data Fig. 2d and Extended Data Table 2). This orthogonal immune profiling approach recapitulated salient features of COVID-19, notably a reduction of 

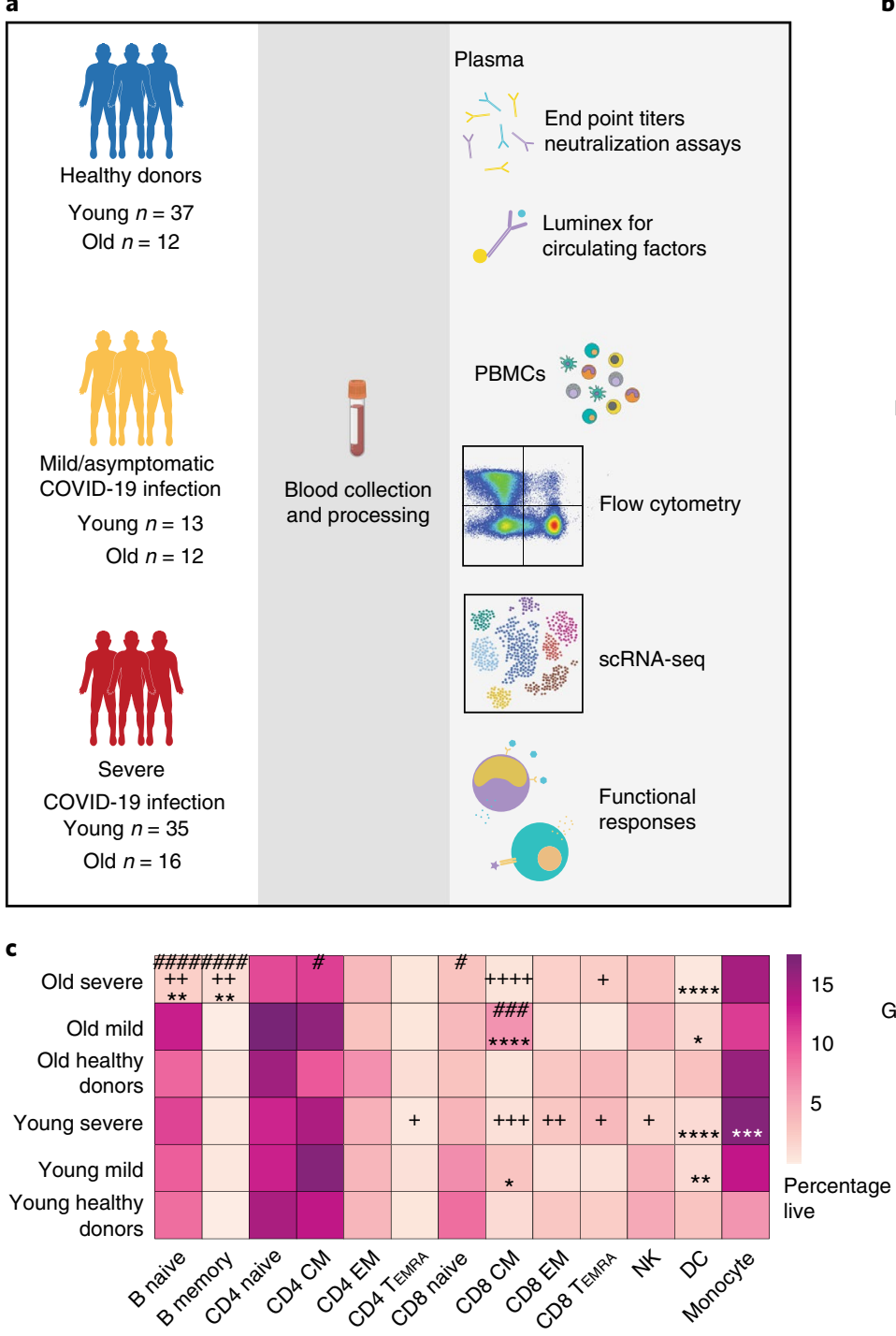

b

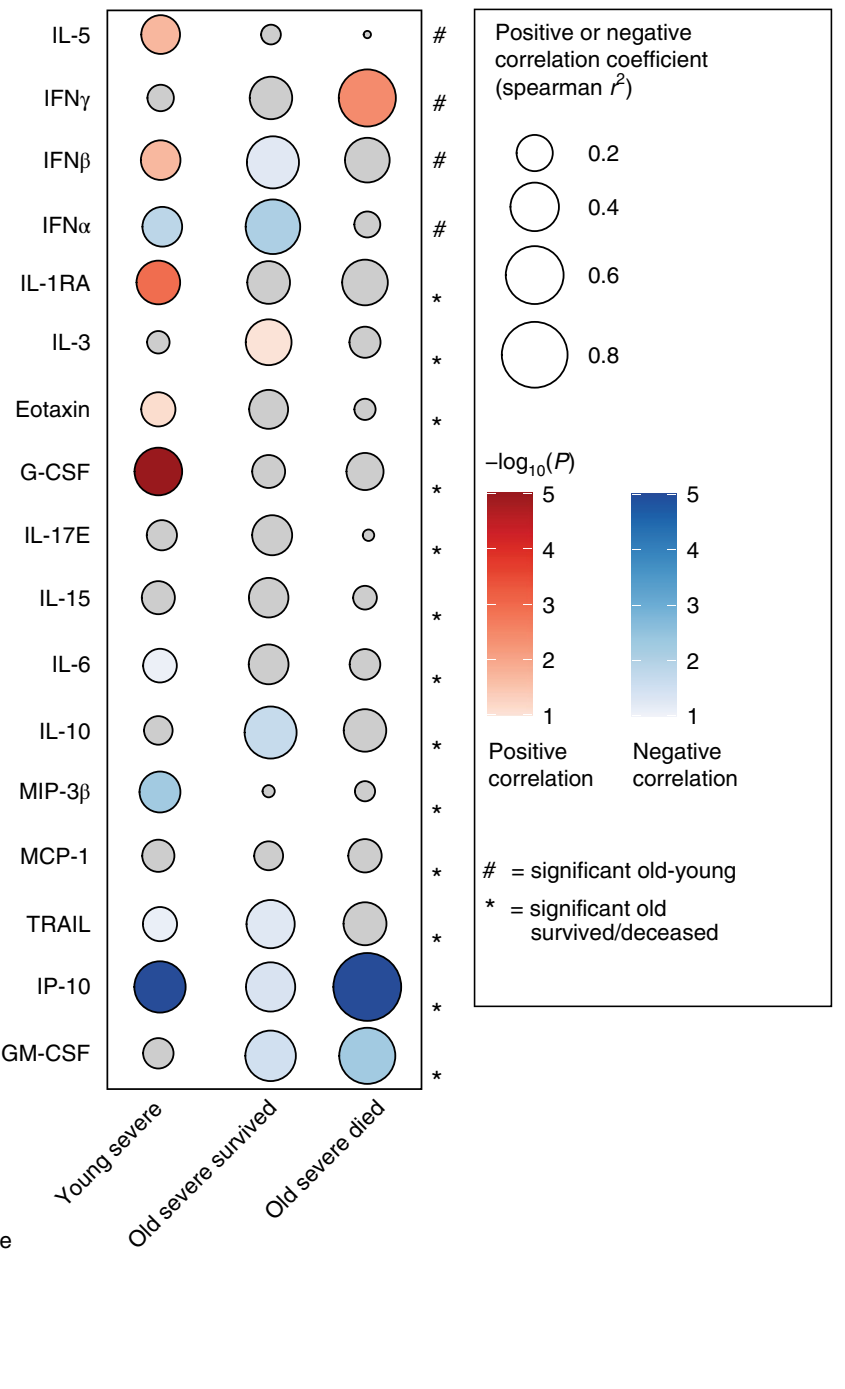

Fig. 1 | Age-dependent alterations in circulating factors and major immune cell subsets with COVID-19. a, Experimental design for the study created partially on Biorender.com. Blood was collected from healthy donors ( $n=49 ; 37$ young and 12 old), mild/asymptomatic patients ( $n=25 ; 13$ young and 12 old) and longitudinally in 35 young and 16 old patients with severe COVID-19. The immune phenotypes of PBMCs and concentration of soluble mediators in plasma were determined using flow cytometry and Luminex, respectively. Longitudinal serological responses to SARS-CoV-2 were measured using ELISA. A subset of PBMC samples ( $n=4$ healthy donors, 4 mild, 4 severe per age group) were profiled using scRNA-seq to determine dynamic peripheral immune adaptations of COVID-19 in mild/severe disease in young and older subjects. b, Bubble plot representing linear regression analyses of the concentration ( $\mathrm{pg} \mathrm{ml}^{-1}$ ) of select cytokines, chemokines and growth factors across DPS. The size of the bubble represents the Spearman correlation coefficient (positive in red and negative in blue) and the color intensity represents the significance of that correlation ( $-\log _{10}(P)$ ) in each of the three groups. Differences with age are denoted with a single asterisk (between old survived and old deceased) or a hash symbol (between young and old) calculated using an ANCOVA test. c, Heat map of proportions of major immune cell subjects derived from live singlets quantified using flow cytometry. Six-way comparisons were tested using a one-way ANOVA with Holm-Šídák multiple comparisons test where * $=$ significant compared to healthy donors, $+=$ significant compared to patients with mild disease, \#=significant difference between old mild/severe and young mild/severe groups where $\star /+/ \# P<0.05, \star \star /++P<0.01, \star \star \star /+++/ \# \# \#<0.001, \star \star \star \star /+++++\# \# \# P<0.0001$.

naive CD8 T cells (especially in older patients) and DCs with severe disease (Extended Data Fig. 2e). Frequency of B cells contracted in young patients but expanded in older patients with severe COVID19 , while frequency of plasmablasts increased in patients with mild disease (Extended Data Fig. 2e). Finally, frequency of natural killer T (NKT cells) increased in both young and old individuals who experienced severe disease (Extended Data Fig. 2e).

Qualitative differences in B cell responses to acute SARS-CoV-2 infection in older patients. To assess the impact of disease severity and age on humoral responses to SARS-CoV-2 infection, we re-clustered the $\mathrm{B}$ cells and plasmablasts and identified five subsets based on the expression of key B cell markers (Fig. 2a and Extended Data Fig. 3a). Modest increases in the proportions of plasmablasts were observed with disease but were more prominent in younger patients with mild disease (Extended Data Fig. 2e). We next measured the frequencies of proliferating $B$ cells using flow cytometry. Independent of age, the frequency of total and proliferating terminally differentiated B cells was increased in patients with severe disease, while that of total and proliferating marginal zone-like B cells was increased only in patients with mild disease (Fig. 2b). 
Differential expression analysis in plasmablasts (Fig. 2c) within each group relative to age-matched healthy donors revealed increased expression in the Ig heavy chain genes IGHG3 and IGHM only in young patients with severe disease (Fig. 2c). Additionally, expression of SSR4, important for B cell effector function, was increased with infection in young patients but decreased in older patients (Fig. 2c). Similarly, expression of the heavy chain gene IGHG3 within memory B cells increased with infection in young patients but decreased in older patients (Fig. 2d). Finally, while severe disease was associated with the induction of genes associated with $\mathrm{B}$ cell activation (CD40, TRAF4, NFKB1/2 and REL), these changes were less pronounced in older individuals (Fig. 2d).

Given the significant age differences in the transcriptional landscapes of memory B cells and plasmablasts after severe COVID-19, we tested for differences in humoral responses with age. SARSCoV-2 Spike-specific IgG and IgA end point titers were measured using ELISA and neutralizing antibodies measured using a focus reduction assay. While no differences were observed in the IgG or IgA end point titers (Fig. 2e and Extended Data Fig. 3b), advanced age was associated with significantly reduced SARS-CoV-2 neutralization titers (Fig. 2f). Moreover, the frequencies of Spike proteinspecific B cells were significantly reduced in older patients with severe disease compared to their young counterparts (Extended Data Fig. 3c and Fig. 2g).

Severe COVID-19 in older patients is associated with increased severity of lymphopenia, exacerbated IFN signaling and dampened antiviral responses. A closer examination of $\mathrm{T}$ cell frequencies revealed a linear drop in total and naive CD4 and CD8 T cells across DPS in older patients (Fig. 3a,b). Severe disease was associated with increased frequencies of proliferating $\mathrm{CD} 4 \mathrm{~T}_{\mathrm{CM}}$ (Fig. 3c) and CD8 effector memory $\mathrm{T}\left(\mathrm{T}_{\mathrm{EM}}\right.$ ) cells (Fig. $3 \mathrm{~d}$ ), although this induction was reduced in the old. In line with the significant loss of naive $\mathrm{T}$ cells and reduced proliferation, plasma levels of the $\mathrm{T}$ cell maintenance factors IL-2 and IL-7 were only significantly increased with DPS in young patients with severe disease (Fig. 3 d). Furthermore, surface expression of IL-2R (CD25) on CD8 T cells was significantly lower in older individuals compared to young individuals with severe disease (Fig. 3e).

We next re-clustered $\mathrm{T}$ and $\mathrm{NK}$ cells to identify subsets at higher resolution (Fig. 3f). This allowed us to identify several subsets of memory CD4 and CD8 T cells as well as NK cells (Fig. $3 \mathrm{f}$ and Extended Data Fig. 4a). Within the CD4 $\mathrm{T}_{\mathrm{EM}}$ subsets, differentially expressed genes (DEGs) with severe COVID-19 enriched predominantly to pathways associated with lymphocyte activation (CD3E, IL2RA, IL7R), response to type I IFNs (IFI6, IRF1), antiviral immunity (ISG15, ISG20, MX1, TRIM8) and positive regulation of cell death (FAS), particularly in the old patients (Fig. 3g). Severe COVID-19 was also associated with an expansion of the CD4 $\mathrm{T}_{\mathrm{EM}}$ cells reexpressing CD45RA ( $\mathrm{T}_{\mathrm{EMRA}}$ ) subset, most notably in young patients (Extended Data Fig. 4b). Moreover, this subset was transcriptionally dysregulated with severe disease only in older patients compared to age-matched controls and older patients with mild disease (Fig. 4a). Specifically, expression of genes associated with ATP metabolism, calcium signaling and response to hypoxia (including cytotoxic molecules such as GZMA, GZMB and KLRD1) were decreased in older patients with mild disease and increased in older patients with severe disease (Fig. 4a,b). Genes that were uniquely downregulated with severe disease in older patients enriched to gene ontology (GO) terms associated with antiviral immunity, leukocyte differentiation and negative regulation of the cell cycle (Extended Data Fig. 4c). A consistent theme across both CD4 $\mathrm{T}_{\mathrm{EM}}$ and $\mathrm{T}_{\text {EMRA }}$ clusters was upregulation of genes that map to $\mathrm{GO}$ terms associated with apoptosis in patients who experienced severe disease, as evidenced by increased scoring of the FAS signaling module in memory CD4 T cells in individuals with severe COVID-19, which was more pronounced in older patients (Extended Data Fig. $4 \mathrm{~d}$ and Supplementary Table 3). Furthermore, a sharper decline in IL-2 signaling signatures was detected in memory CD4 T cells from older individuals with severe disease only (Extended Data Fig. 4e).

Single-cell analysis of CD8 T cells identified four memory subset clusters in addition to naive CD8 T cells (Fig. $3 \mathrm{f}$ and Extended Data Fig. 4a). These memory clusters were identified as CD8 IFN (expressing high levels of IFN-simulated genes such as IFIT2, IFIT3), activated memory CD8 (CD69 ${ }^{\text {hi }}$ expressing CCL4 and IFNG) and two subsets of cytotoxic CD8 T cells (GZMH $H^{\mathrm{hi}}$ and $\left.G Z M K^{\mathrm{hi}}\right)$ (Fig. $3 \mathrm{f}$ and Extended Data Fig. 4a). Comparisons of COVID-19 associated DEGs in memory CD8 $\mathrm{T}$ cells revealed upregulation of prosurvival $(B C L 2)$ and antiviral factors $(O A S 3, T X N I P)$ with mild disease and enrichment of factors associated with the tissue-resident CD8 T cell development program (RUNX3, VIM, TGFB1) in severely affected patients (Fig. 4c). More importantly, while CD8 T cells from both young and old patients with severe COVID-19 expressed elevated levels of chemokines (CCL4L2, XCL2) and cytotoxic molecules (PRF1, GZMB, KLRD1) (Fig. 4c), the overall cytotoxicity scores in the $G Z M K^{\text {hi }}$ clusters were significantly higher in older patients (Extended Data Fig. 4f). As observed in the CD4 $\mathrm{T}_{\mathrm{EM}}$ cluster, severe disease was associated with signatures of heightened CD8 $\mathrm{T}$ cell activation $(C D 3 D$ and $C D 8 B)$ (Fig. 4 c). Finally, our analyses revealed significant upregulation of IFN signaling module scores in all CD8 T cell clusters with mild disease in both young and old individuals (Extended Data Fig. 4g), albeit to a greater extent in young patients $(P<0.0001)$. In contrast, IFN signaling module scores were significantly higher in older patients relative to young patients with severe disease $(P<0.0001)$ (Extended Data Fig. $4 \mathrm{~g})$ peaking at DPS 18 in the CD8 IFN cluster (Extended Data Fig. 4h).

We next investigated if these transcriptional changes were associated with altered antigen-specific $T$ cell responses against SARSCoV-2 (Fig. 4d). To quantify antigen-specific responses, purified $\mathrm{CD} 2+$ cells (with $<2 \%$ contaminating antigen-presenting cells) were

Fig. 2 | Qualitative differences in SARS-CoV-2-specific B cell responses with age. a, UMAP projection of 6,334 B cells and plasmablasts with major subsets annotated. $\mathbf{b}$, Dot plots showing the frequency of total (top) and Ki67-expressing (bottom) terminally differentiated memory B cells (left) and marginal zone-like B cells (right) determined from flow cytometry ( $n=12$ young healthy donors, 12 older healthy donors, 6 young mild, 4 old mild, 27 young severe and 8 old severe). All percentages are reflective of the fraction of total live cells. c, Bubble plot showing the expression of selected genes in the plasmablast subset. The color of the dot represents the average expression and the size represents the percentage of cells expressing that gene. d, Bubble plot showing the expression of selected genes in memory B cell subsets. The color of the dot represents the average expression and the size represents the percentage of cells expressing that gene. e, Bar plots of Spike-specific IgG end point titers from patients with severe COVID-19 ( $n=12$ young from 7 individuals, $n=22$ old from 16 individuals). f, Bar plots of antibody neutralization of SARS-CoV-2 virus (half-maximal inhibitory concentration $\left.\left(\mathrm{IC}_{50}\right)\right)$ measured from the plasma of patients with severe COVID-19 ( $n=13$ young from 6 individuals, 22 old from 12 individuals; exact $\left.P=0.014\right)$. $\mathbf{g}$, Bar plots showing the percentage of Spike $B$ cells in the PBMCs of patients with severe COVID-19 ( $n=9$ per age group; exact $P=0.036)$. Two-group differences were tested using a two-sided unpaired $t$-test with Welch's correction. Six-way comparisons were tested using a one-way ANOVA with HolmŠídák multiple comparisons test where ${ }^{*}=$ significant compared to healthy donors, $+=$ significant compared to mild patients, \#=significant difference between old mild/severe and young mild/severe groups. The error bars represent median values with the interquartile range $(\mathrm{IQR}) . P$ values: ${ }^{*}+/ / \# P<0.05$, ${ }^{\star * /++} P<0.01,{ }^{\star \star * /+++} P<0.001$. 
stimulated with overlapping peptide pools covering several SARS$\mathrm{CoV}-2$ open reading frames (E/M/N/Nsp14/ORF3a/6/7/8/10) for $24 \mathrm{~h}$ and secreted cytokine levels were assessed using Luminex (Fig. 4e). T cells from older patients with severe COVID-19 secreted attenuated levels of IFN $\gamma(P=0.09)$ and IL-2 $(P=0.1)$ relative to young patients with severe disease while the responses to CD3 were comparable between the two groups (Fig. 4e) suggesting impaired antigen-specific responses with age. Flow cytometry a
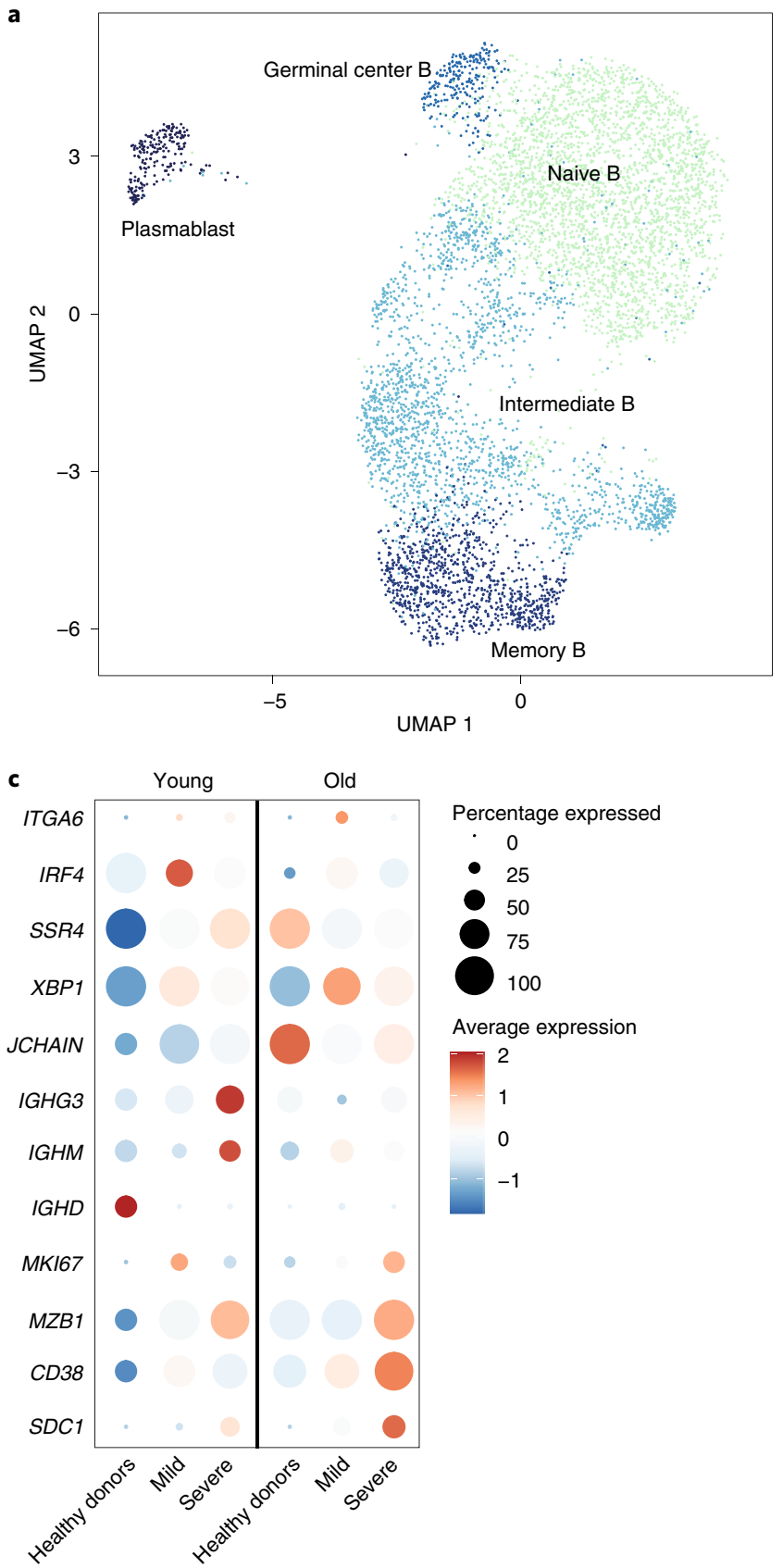

e

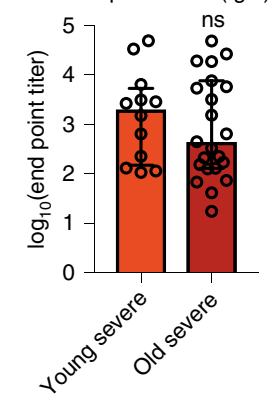

Percentage expressed

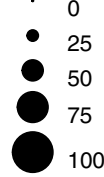

Average expression

2

0

0
-1
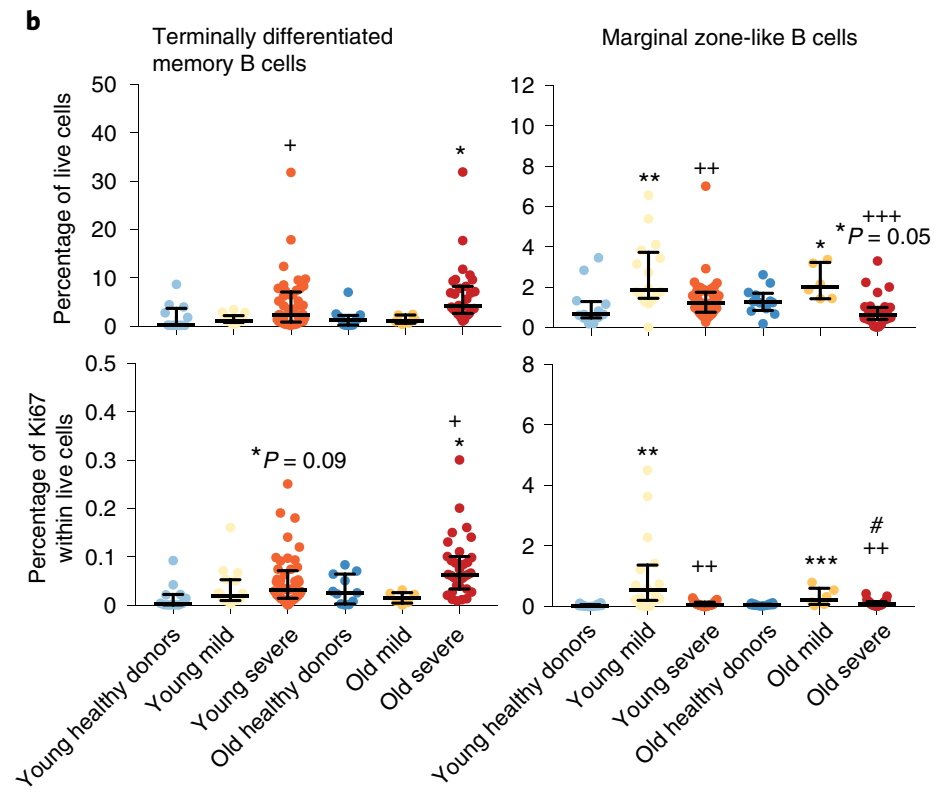

d
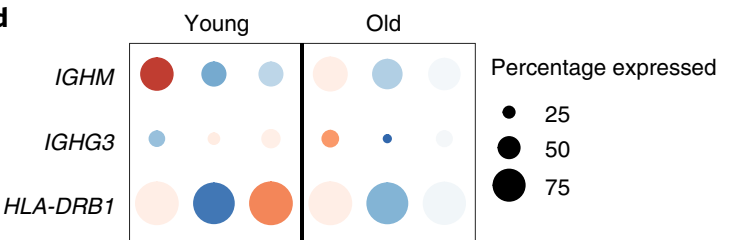

$C D 40 \bigcirc \bigcirc \quad$ Average expression
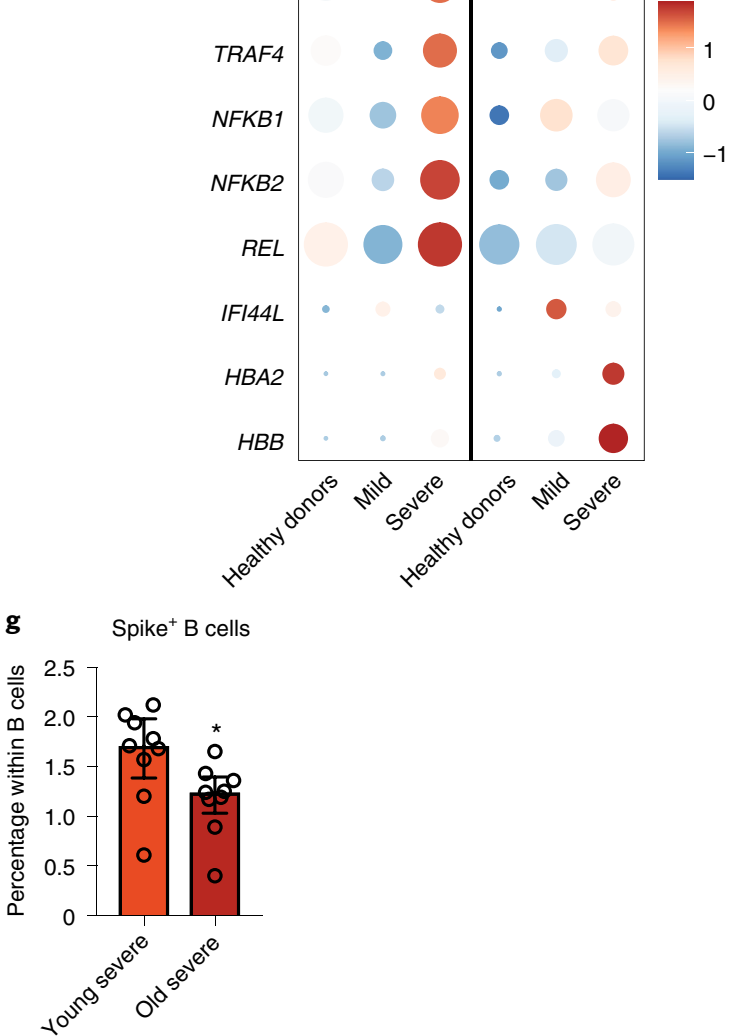


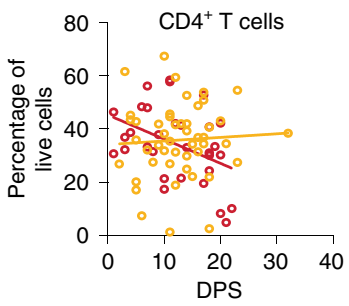

Old: $r^{2}=-0.4 ; P=0.02$

Young: $r^{2}=0.05 ; P=0.7$

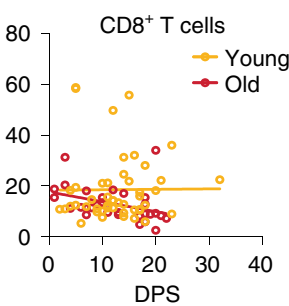

Old: $r^{2}=-0.5 ; P=0.003$

Young: $r^{2}=0.1 ; P=0.5$

c

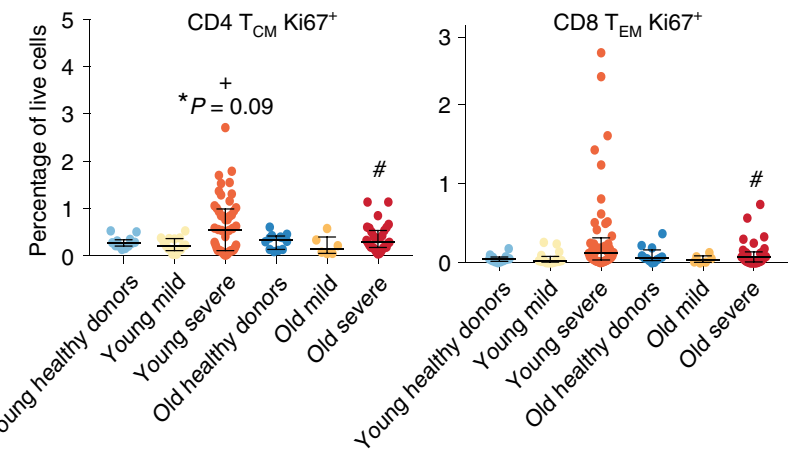

f

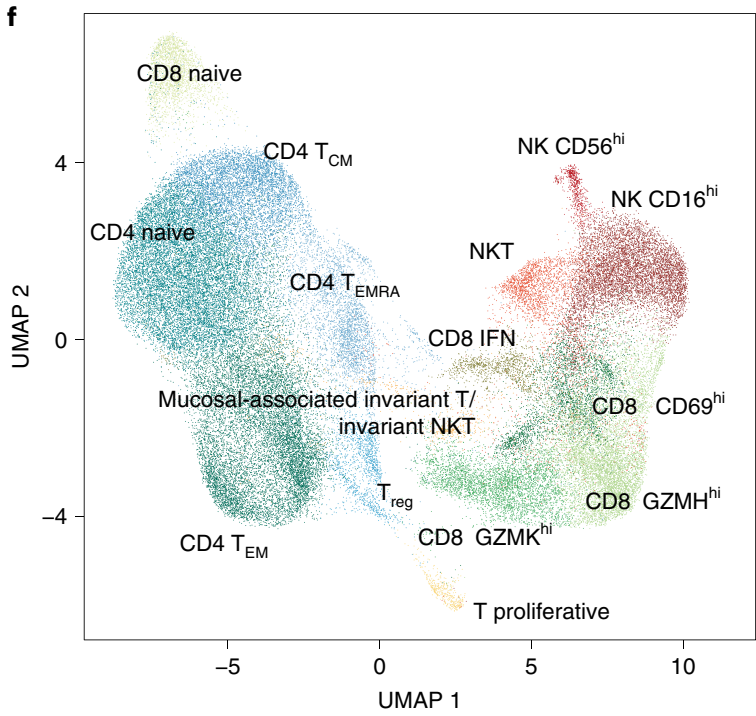

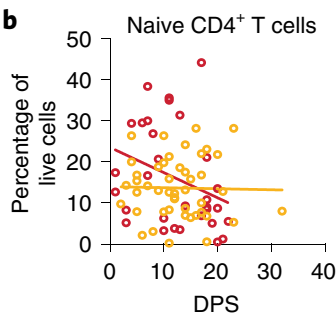

Old: $r^{2}=-0.36 ; P=0.04$

Young: $r^{2}=-0.05 ; P=0.7$

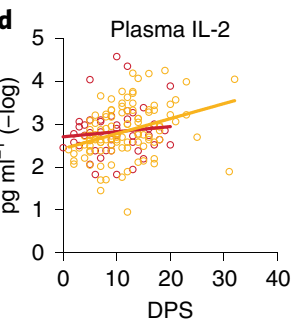

Young : $r^{2}=0.38 ; P<0.0001$ Old : $r^{2}=0.08 ; P=0.55$

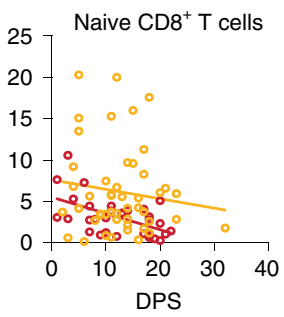

Old: $r^{2}=-0.49 ; P=0.005$

Young: $r^{2}=-0.04 ; P=0.7$
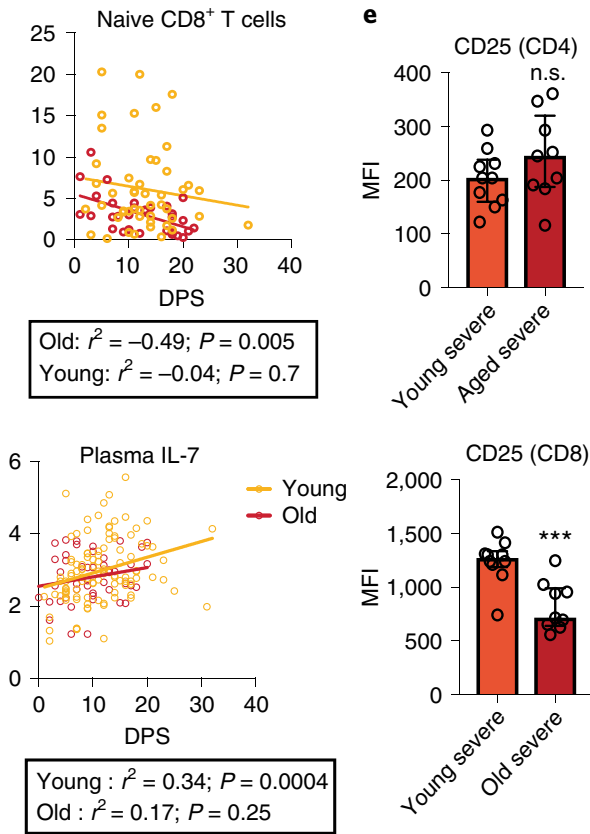

g

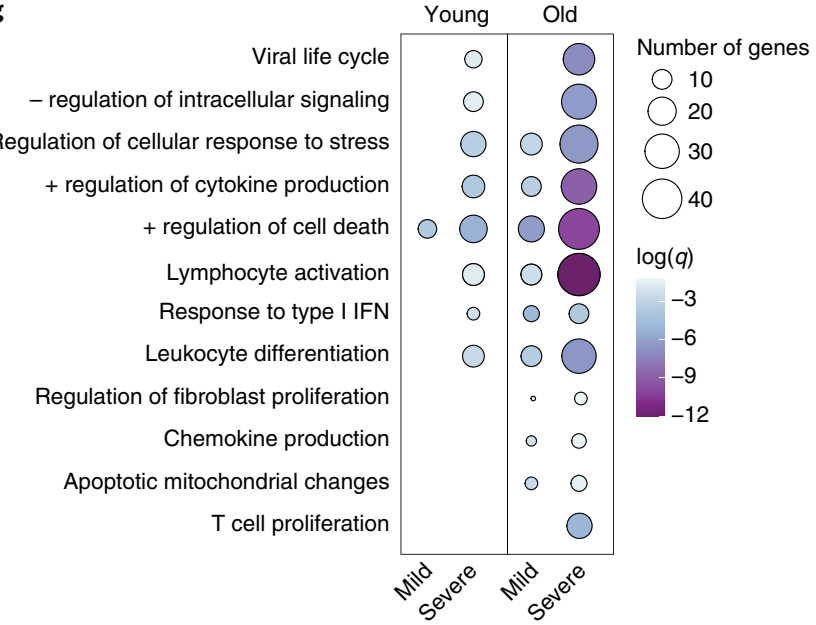

Fig. 3 | Heightened activation and cytotoxicity of T cells from older patients with severe COVID-19. a,b, Linear regression analysis of frequencies of total (a) and naive T cell (b) subsets with DPS in young and older individuals. c, Dot plots comparing the frequencies of proliferating CD4 $\mathrm{T}_{\mathrm{CM}}$ (left) and proliferating CD8 $T_{E M}$ (right) cells with mild and severe COVID-19 in young and older individuals measured using flow cytometry ( $n=12$ young healthy donors, 12 old healthy donors, 6 young mild, 4 old mild, 27 young severe, 8 old severe). d, Linear regression of plasma IL-2 and IL-7 levels and DPS within young (yellow) and older individuals (red) with severe COVID-19. The $P$ value on the graph refers to ANCOVA testing of differences with age. The Spearman correlation coefficient and corresponding significant $P$ for DPS is highlighted under each graph. e, Bar plots showing the mean fluorescence intensity (MFI) of CD25 expression on CD4 and CD8 T cells from patients with severe COVID-19 ( $n=10$ young, 9 old; exact $P=0.0007$ ). f, UMAP projection of 66,656 lymphocytes re-clustered from the main UMAP to identify the T and NK cell subpopulations at a higher resolution. Major subsets

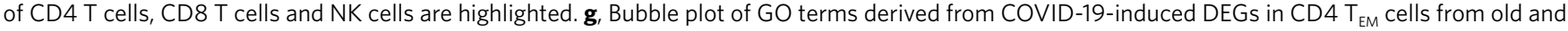
young individuals. The color and size of the bubble represents statistical significance and the number of genes, respectively. Two-group differences were tested using a two-sided unpaired $t$-test with Welch's correction. Six-way comparisons were tested using a one-way ANOVA with Holm-Šídák multiple comparisons test where ${ }^{*}=$ significant compared to healthy donors, $+=$ significant compared to patients mildly affected, \# $=$ significant difference between old mild/severe and young mild/severe groups. The error bars represent the median values with IQR. ${ }^{+/ \#} P<0.05,{ }^{\star \star \star} P<0.001$.

analysis of the unstimulated $\mathrm{T}$ cells after $24 \mathrm{~h}$ in culture showed decreased frequencies of $\mathrm{CD} 69^{+} \mathrm{CD}_{40 \mathrm{~L}^{+}}$and $\mathrm{OX} 40^{+} \mathrm{CD} 40 \mathrm{~L}^{+} \mathrm{T}$ cells in older individuals with severe COVID-19 (Fig. 4f). Furthermore, after peptide stimulation, older individuals had fewer antigenspecific CD4 $\left(\mathrm{OX} 40^{+} \mathrm{CD} 137^{+}\right)$and $\mathrm{CD} 8\left(\mathrm{CD} 69^{+} \mathrm{CD} 137^{+}\right) \mathrm{T}$ cells (Fig. 4g).
Enrichment of terminally differentiated NK cells with an exhausted phenotype in older individuals with severe COVID-19. Given their critical role in antiviral immunity during acute infection, we next examined the impact of COVID-19 disease severity and age on NK cell phenotypes. Two major populations of NK cells were identified by scRNA-seq based on the expression of FCGR3A 

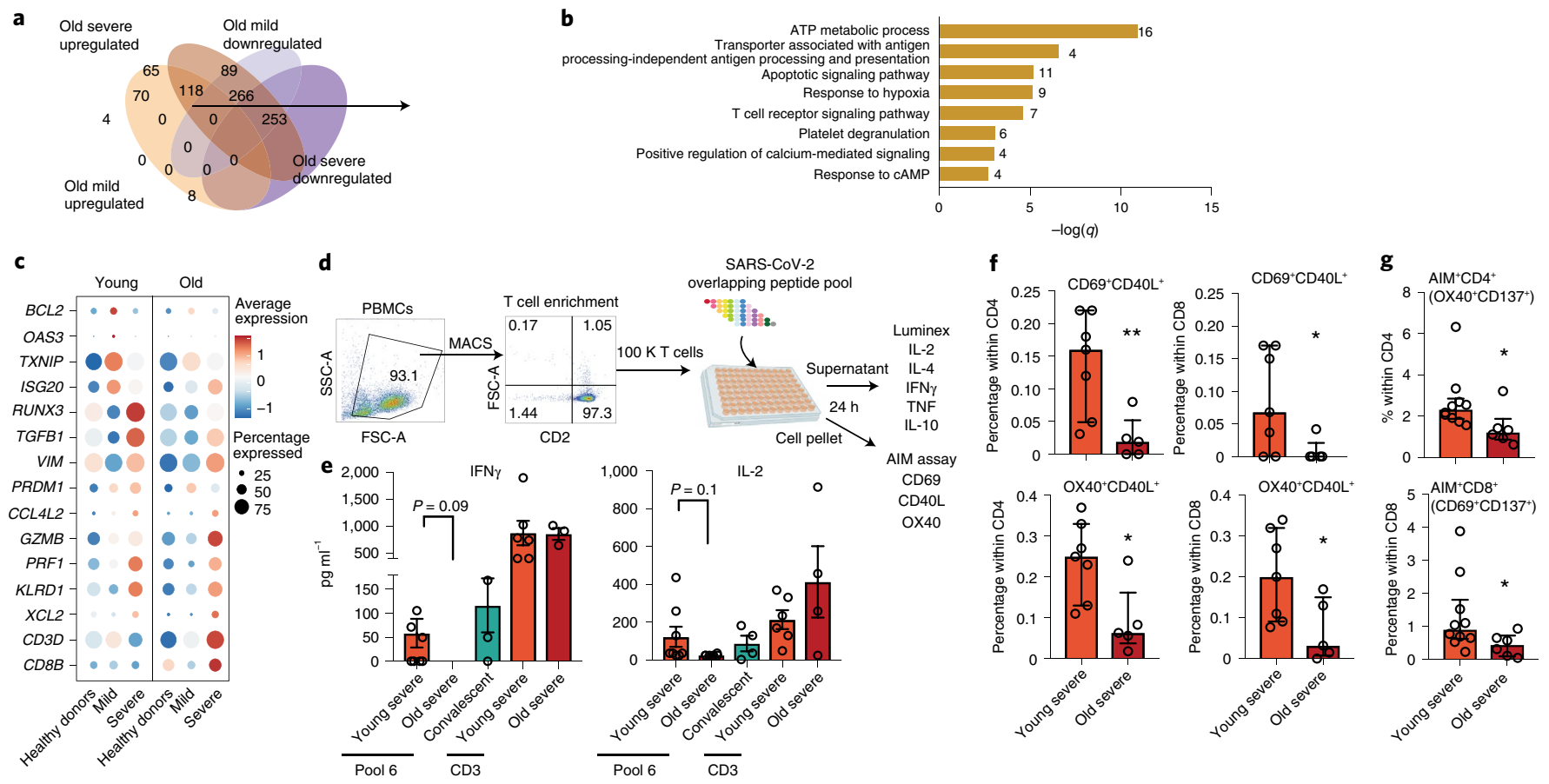

Fig. 4 | Age-associated disparities in T cell activation and antigen-specific T cell responses in severe COVID-19. $\mathbf{a}$, Venn diagram of DEGs detected in CD4 $T_{\text {EMRA }}$ clusters in older individuals with mild and severe COVID-19. b, Bar graph showing GO enrichment of DEGs upregulated with severe COVID19 but downregulated in older individuals with mild COVID-19 within the CD4 T EMRA cluster. The gene numbers associated with each term are indicated next to each term. The size of the bar indicates the significance of the enrichment. $\mathbf{c}$, Bubble plot of a subset of genes that are differentially responsive in mild versus severe COVID-19 within memory CD8 T cell clusters. The size of the bubble is indicative of the percentage of cells expressing the marker and the color indicates the average expression level ranging from low (blue) to high (red). d, T cells from young and old patients with severe COVID-19 were purified by magnetic-activated cell sorting positive selection beads and stimulated with SARS-CoV-2 overlapping peptides. e, Secretion of selected immune mediators was measured by Luminex where the bar graphs show pg ml-1 concentrations of IFN $\gamma$ and IL- 2 in ( $n=8$ young severe, 6 old severe, 4 convalescent) patients after stimulation with peptide pool 6 or CD3 positive control. f, Percentages of $\mathrm{CD}_{69}{ }^{+} \mathrm{CD}_{40 \mathrm{~L}^{+}}$(top) or OX40+CD4OL ${ }^{+}$(bottom) within total CD4 (left) or CD8 (right) from patients with severe COVID-19 ( $n=10$ young, 9 old). g, SARS-CoV-2-specific CD4 and CD8 T cells measured as percentage of $\mathrm{AIM}^{+}\left(\mathrm{OX} 40^{+} \mathrm{CD} 137^{+}\right) \mathrm{CD} 4 \mathrm{~T}$ cells (top) and $\mathrm{AIM}^{+}\left(\mathrm{CD} 69^{+} \mathrm{CD} 137^{+}\right) \mathrm{CD} 8 \mathrm{~T}$ cells (bottom) after T cell stimulation with peptide pool 6 ( $n=10$ young severe, 8 old severe). Two-group comparisons were tested using a two-sided unpaired $t$-test with Welch's correction. The error bars represent the median values with the IQR. ${ }^{\star} P<0.05,{ }^{\star \star} P<0.01$.

(CD16) and NCAM1 (CD56) (Fig. 3fand Extended Data Fig. 5a). We also identified a cluster of cytotoxic NK cells expressing high levels of PLGC2 (NKT cells) that drive innate immune responses against virally infected cells ${ }^{39}$. The frequency of this subset increased significantly in young patients and only modestly in older patients with severe disease (Extended Data Fig. 5b). NK cell exhaustion module scores from the NCAM $1^{\text {hi }}$ cluster were increased with severe disease and with advanced age (Fig. 5a). In contrast, induction of a robust cytotoxic program was only evident in young patients with severe disease as indicated by a significant increase in $\mathrm{CD} 56^{++}$granzymeB ${ }^{+}$ cells by flow cytometry (Fig. 5b). This observation is in line with increased levels of granzyme B in plasma from young, but not older patients, with severe disease (Extended Data Fig. 1a). On the other hand, the frequency of NK cells expressing the activation marker KLRG1 and/or terminal differentiation marker CD57 was significantly increased in older patients with severe disease compared to their young counterparts (Fig. 5c).

Even though the frequencies of the FCGR3A $A^{h i}$ NK cell cluster did not change with disease severity/age, we observed significant changes in their transcriptional program with COVID-19. DEGs from each disease group compared to their age-matched healthy donors enriched to GO processes associated with leukocyte activation, cell death and response to virus (Extended Data Fig. 5c). Interestingly, the pathways that were upregulated with severe disease in older patients (lymphocyte activation and leukocyte activation in immune response) were strongly downregulated with mild infection in young patients.
Indeed, genes involved in cell migration (LGALS3 and CCL5) and activation (HLA-DRB1) were highly upregulated with severe disease in older patients (Fig. 5d). On the other hand, expression of numerous IFN-stimulated genes (ISGs) (for example, ISG20, IFIT3, IFITM1) as well as B2M, IRF7 and STAT1 were upregulated with mild disease (Fig. 5d). Indeed, the IFN module score was highest in NK cells from patients with mild disease (Fig. 5e). However, and as described for T cells, the IFN module score was higher in NK cells from old compared to young patients with severe disease in both CD56 ${ }^{\text {hi }}(P<0.05)$ and $\mathrm{CD}_{16}{ }^{\mathrm{hi}}(P<0.0001)$ clusters (Fig. 5e). Finally, the expression of genes associated with cytokine signaling (TNFSF4, TNFRSF18, STAT3, TNF, $M A P 3 K 8, R E L A)$ and several cytokines and chemokines and their receptors (CXCR4, IFNGR1, IL21R, IL4R) were upregulated with severe disease but to a less extent in older patients (Fig. $5 \mathrm{~d}$ ).

Aging exacerbates regulatory skewing of monocytes and DCs in patients with severe COVID-19. Young patients showed an increase in both classical and nonclassical monocytes (Extended Data Fig. 6a). On the other hand, the frequency of classical monocytes was significantly decreased while that of nonclassical monocytes was increased in older patients (Extended Data Fig. 6a). Regardless of age, the frequencies of plasmacytoid and myeloid DCs was reduced in patients with COVID-19 in a severity-dependent manner (Fig. 1c and Extended Data Fig. 6b). The most striking change, however, was the downregulation of surface HLA-DR and activation marker CD86 on both monocyte (Fig. 6a and Extended Data Fig. 6c) and 

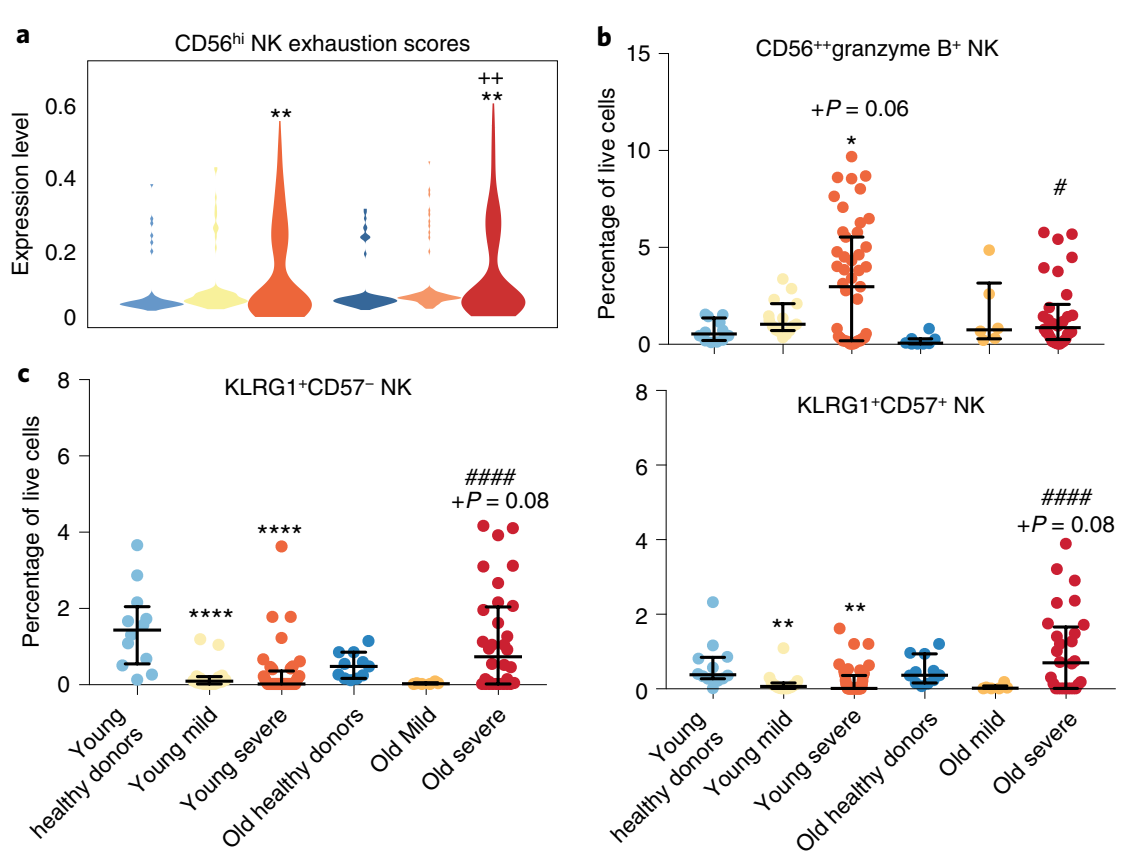

d

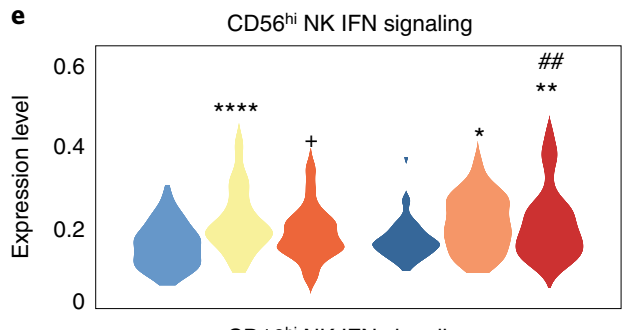

CD16 ${ }^{\text {hi }}$ NK IFN signaling

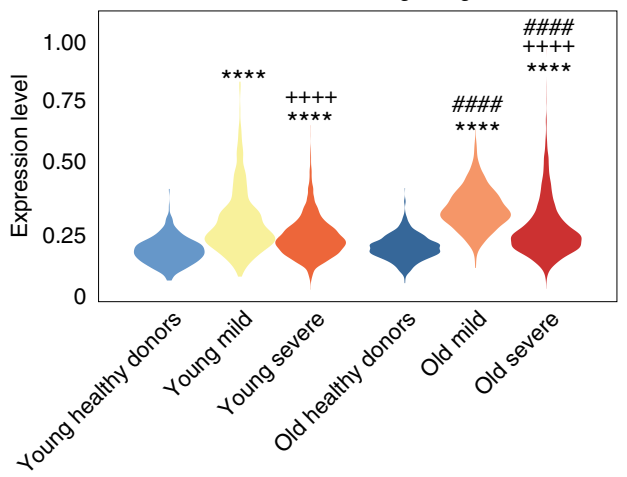

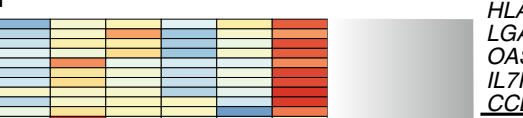

GALS3

OASL

CCL5

B2M

CFL1

CEBPB

HLA-DPA1

ISG20

IKZF1

IFIH1

IFIT3

IFI16

IRF7

STAT1
HLA-DPB1

IFIT1

OAS3

IFI44

IFI6

IFITM1

IFITM2

IFITM3

SOCS1

IFIT2

IFIT2

ISG15

PLCG2

IKZF3

TNFRSF

STAT3
CXCR4

CXCR4
MAP 3 K 8

HLA-E

TNF

CD44

IL4R

RELB

RELB

BCL3

$H S P D 1$

CD83
TNFAIP3

ICAM1

SMAD3

RELA

GATA3

GATA3

CD55
TNFSF14

APOBEC $3 G$

LAG3

CD74

TNFRSF4

TNFRSF1B

PIK3R1

IFNGR1

IRF4

RUNX3

IL21R

TGFB1

Fig. 5 | Increased frequency of terminally differentiated NK cells with an exhausted phenotype in older individuals. a, Violin plots comparing the exhaustion module scores for CD56 ${ }^{\text {hi }}\left(N_{C A M}{ }^{\text {hi }}\right)$ NK cell subset. b,c, Dot plots comparing the frequencies of granzyme B-expressing CD56 $6^{++} \mathrm{NK}$ cells (b) or KLRG1+ NK cells expressing CD57 (c) measured using flow cytometry ( $n=12$ young healthy donors, 12 older healthy donors, 6 young mild, 4 old mild, 27 young severe, 8 old severe). d, Clustered heat map comparing normalized expression of genes enriching to the GO term 'lymphocyte activation' from the CD16 ${ }^{\text {hi }}$ NK subset obtained from patients with mild and severe COVID-19. The colors represent scaled gene expression ranging from blue (low) to red (high). Selected genes are highlighted. The error bars represent the medians and IQR. e, Violin plots comparing IFN signaling module scores in the CD56 hi and CD16 ${ }^{\text {hi }}$ NK cell subsets. Six-way comparisons were tested using a one-way ANOVA with Holm-Šídák multiple comparisons test where ${ }^{*}=$ significant compared to healthy donors, $+=$ significant compared to mild patients, \#= significant difference between the old mild/severe and young mild/severe groups. ${ }^{\star /+/ \#} P<0.05,{ }^{\star \star /++/ \# \# ~} P<0.01$, ${ }^{\star \star \star \star /++++/ \# \# \# \# ~} P<0.0001$.

DC subsets (Fig. 6b and Extended Data Fig. 6c). Furthermore, HLA-DR downregulation was more severe on cells from older patients compared to young patients (Fig. 6a,b) and regression analysis of HLA-DR expression over DPS revealed a recovery of its expression on classical monocytes over the course of infection in young but not older individuals $(P<0.0001)$ (Fig. 6 c). On the other hand, expression of CD86 was relatively higher in older individuals with severe disease compared to young individuals (Fig. 6a,b).

Re-clustering of the myeloid cell subsets from the scRNA-seq data identified four clusters of classical monocytes (MS1-MS4), 

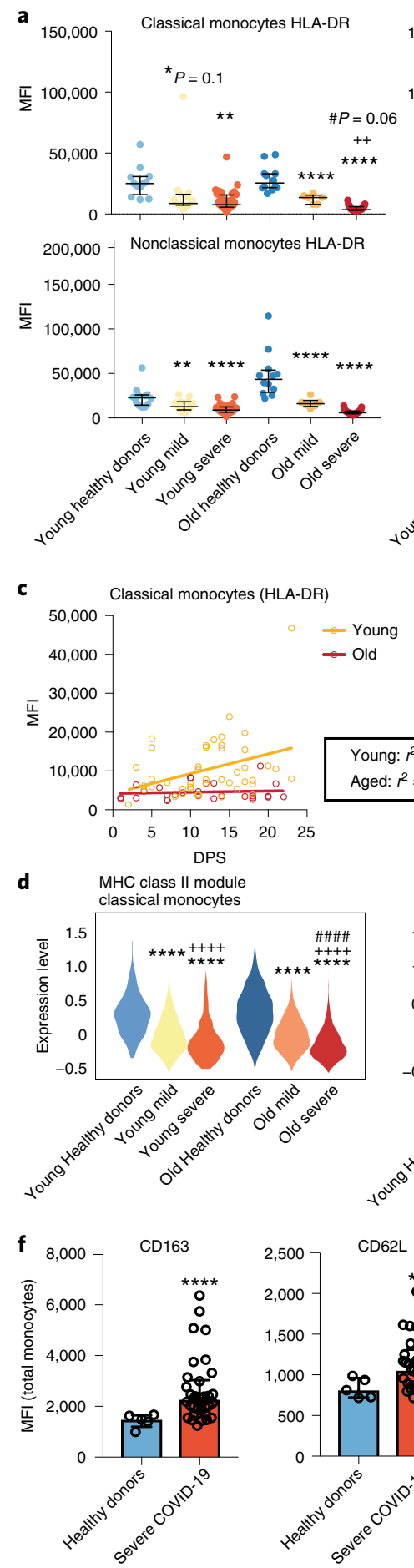

- Young
- Old

Young: $r^{2}=0.32 ; P=0.03$

Aged: $r^{2}=0.07 ; P=0.6$

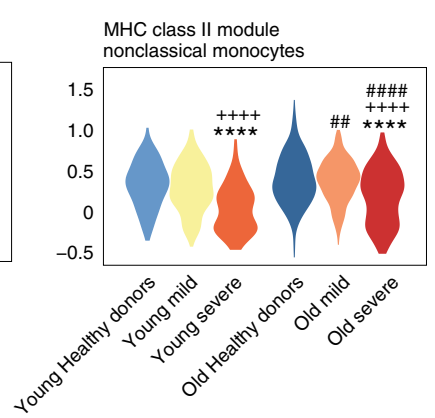

CD62L
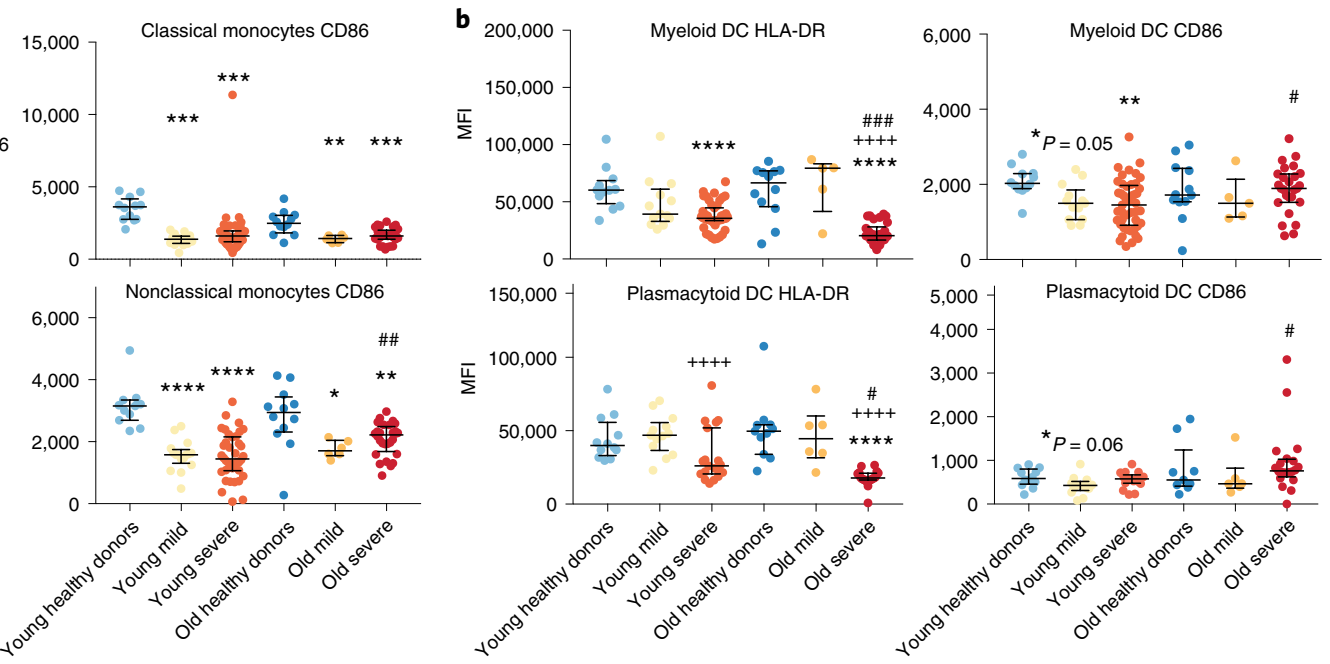

e

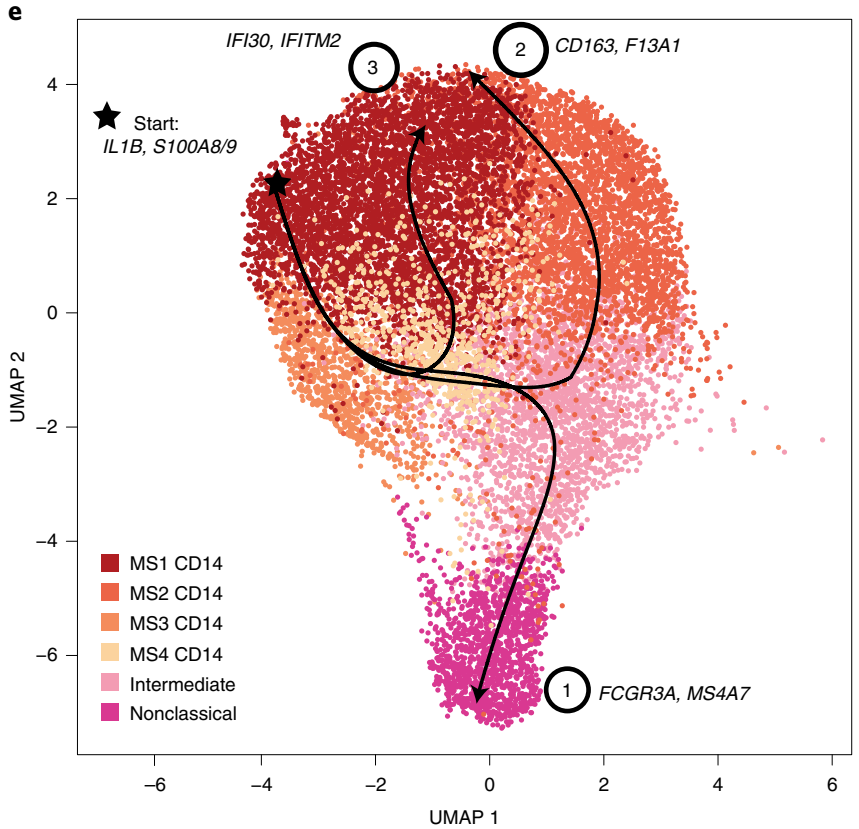

Fig. 6 | Myeloid cell adaptations with age and severe COVID-19. a,b, Dot plots comparing the MFIs of HLA-DR and the co-stimulation marker CD86 in classical and nonclassical monocytes (a) and myeloid and plasmacytoid DCs (b) ( $n=12$ young healthy donors, 12 old healthy donors, 6 young mild, 4 old mild, 27 young severe and 8 old severe). c, Linear regression analysis of HLA-DR surface expression on classical monocytes as a function of DPS in young and older individuals. The Spearman correlation coefficient and corresponding significant $P$ value for DPS are highlighted. d, Violin plot comparing changes in $\mathrm{MHC}$ class II RNA module scores within classical and nonclassical monocytes. e, UMAP projection of 15,922 monocytes (classical and nonclassical) clustered from the main UMAP. The three trajectories identified by Slingshot are highlighted with markers describing each trajectory. f, Surface expression changes in CD163 and CD62L on total monocytes from healthy donors $(n=5)$ and patients with severe COVID-19 ( $n=35$ samples from 15 individuals; exact $P=<0.0001$ left, exact $P=0.0015$ right). The error bars represent the medians and IQR. Two-group comparisons were tested using a two-sided unpaired t-test with Welch's correction. Six-way comparisons were tested using a one-way ANOVA with Holm-Šídák multiple comparisons test where ${ }^{\star}=$ significant compared to healthy donors, $+=$ significant compared to patients with mild disease, \#=significant difference between old mild/severe and

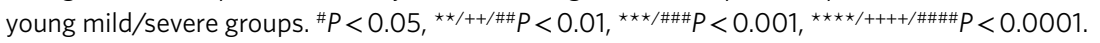


intermediate and nonclassical monocytes, as well as myeloid and plasmacytoid DCs based on the expression of canonical markers (CD14, FCGR3A, FCER1A, LILRA4) (Extended Data Fig. 6d,e). Age-dependent differences in surface HLA-DR on monocytes were validated at the transcriptional level since major histocompatibility complex (MHC) class II module scores were reduced with infection in a severity- and age-dependent manner $(P<0.0001)$ (Fig. $6 \mathrm{~d})$. Trajectory analysis revealed three unique lineages within the monocyte subsets (Fig. 6e) starting from the classical MS1 subset and ending either in nonclassical monocytes (lineage 1), antiviral cluster-expressing ISGs (lineage 3) or regulatory monocytes (lineage 2) expressing CD163 and F13A1 (Fig. 6e). This regulatory lineage was more prominent in patients with severe disease and was confirmed by flow cytometry with increased surface expression of CD163 and CD62L (Fig. 6f). Additionally, surface expression of CX3CR1 and CD11b was downregulated and remained reduced, whereas both CCR5 and CD64 increased over time with acute infection (Extended Data Fig. 6f).

Severe COVID-19 paralyzes innate immune responses to secondary stimulation. Differential gene expression analysis within classical monocytes revealed significant enrichment to the GO terms 'response to virus' and 'response to IFN- $\gamma$ ' in all patients, which was most significant in patients with mild disease. Interestingly, genes associated with negative regulation of immune response were differentially expressed only in the older group (Extended Data Fig. 7a). We next examined the impact of age and disease severity on signaling modules within myeloid cells (Fig. 7a). Within classical monocytes expression of several ISGs and other viral response markers was significantly increased in both young and older patients with mild disease (Extended Data Fig. 7b). Expression of alarmin molecules (S100A8, S100A9, S100A12) was increased in older patients with severe disease while that of antigen presentation was decreased (HLA-DRA) in classical monocytes in individuals with severe disease (Extended Data Fig. 7b). Finally, significant changes in the expression of genes associated with wound healing, myeloid cell activation and hematopoiesis were observed in nonclassical monocytes in individuals with both mild and severe disease in older patients (Extended Data Fig. 7c). DEG analysis in the myeloid DC subset revealed significantly increased expression of the IFN-responsive genes ISG20, MX1, STAT1 and IFI44L with disease in older patients (Extended Data Fig. 7d). Retinoic acidinducible gene I (RIG-I) signaling was also increased in the myeloid DC population from older patients with only a modest increase in young patients (Extended Data Fig. 7e).

We next examined changes in pro-inflammatory and antiviral signaling modules in myeloid cell subsets with age and disease severity. While NF- $\kappa \mathrm{B}$ signaling was downregulated in the monocytes of individuals with both mild and severe disease, its suppression was more prominent in older individuals with severe disease $(P<0.0001)$ (Fig. 7a). In contrast, type I IFN signaling scores were elevated in monocytes and DCs of individuals with both mild and severe disease, with a higher magnitude in older individuals with severe disease (Fig. 7a). To uncover the functional consequences of the transcriptional changes, PBMCs (healthy donors $n=4$; severe $n=15$ ) were stimulated with either a viral or bacterial agonist cocktail and the production of IL- 6 , TNF and IFN $\alpha$ by monocytes and DCs were measured using flow cytometry (Fig. 7b). In response to either bacterial or viral agonists, a lower percentage of both monocytes and DCs in patients with severe disease produced IL-6 and TNF, regardless of age (Fig. 7c). We also detected a positive correlation between IFN $\alpha$ production and DPS in patients with severe disease (Fig. 7d). These data suggest that severe COVID-19 leads to a rewiring of the inflammatory response pathways in myeloid cells with implications for response to secondary infections.

\section{Discussion}

The clinical presentation of COVID-19 is highly heterogeneous; it is influenced by host factors such as age, sex, body mass index and underlying medical conditions, notably diabetes, hypertension and cardiovascular disease. Advanced age is a major driver of severe disease with individuals older than 50 accounting for $95 \%$ of deaths and exhibiting exponential increase in hospitalization and intensive care unit (ICU) admissions ${ }^{8}$. This increased vulnerability of older individuals to respiratory viral infections is mediated by age-associated deterioration of immune function (immunosenescence) and heightened inflammation at baseline (inflammaging) ${ }^{19}$. While recent studies have shed light on host response to mild and severe COVID-19, the precise immune correlates of the disease in at-risk populations, particularly older adults, are unclear. To address this question, we used a systems immunology approach to profile the circulating inflammatory environment using Luminex, frequency and phenotype of key immune cells using flow cytometry, and transcriptional landscape using scRNA-seq in PBMC samples from young and old patients with mild and severe COVID-19 and compared these measurements to those obtained in age-matched healthy donors.

Shifts in plasma inflammatory environment and the ensuing cytokine storm in severe COVID-19 have been well documented ${ }^{22,24}$. Our analysis, however, revealed that older individuals with severe disease showed significantly higher induction of GM-CSF, TRAIL, IP-10 and IL-10 but lower levels of granzyme B and RANTES compared to their younger counterparts. Lower levels of RANTES coupled with higher levels of IP-10 indicate dysregulated recruitment of T cells with potentially greater adhesion to endothelial cells ${ }^{40-42}$. Furthermore, linear regression analysis revealed a failure to upregulate IFN $\beta$ in older individuals but a progressive increase in IFN $\gamma$ levels in older individuals who succumbed to the disease. On the other hand, young individuals exhibited more robust increases in both $\mathrm{T}$ cell factors (IL-2, IL-7, TNF, IL-4, IL-5) and myeloid factors such as IL- $1 \alpha$ and IL- $1 \beta$. Levels of IL-1RA increased with DPS in young but not older patients while the converse was true for GM-CSF, a myelopoietic pro-inflammatory growth factor ${ }^{43}$. These data suggest discordant inflammatory and regulatory responses in older individuals with severe COVID-19, particularly in non-survivors. Interestingly, lower levels of IL-17 during the first five DPS was associated with poor prognosis (ICU admission/intubation and death). This observation differs from recent reports that high levels of IL-17 (ref. ${ }^{22}$ ) and $\mathrm{T}$ helper 17 cells $^{44}$ are correlated with the immunopathology of severe COVID-19 and the proposed use of IL-17 blockade as a therapeutic avenue to limit acute lung injury ${ }^{45}$. This discrepancy highlights the need for careful evaluation of the timing for administering immune modulators in the context of COVID-19.

As reported previously, we observed substantial induction of plasma B cells with mild disease $\mathrm{e}^{30,46-48}$, albeit less prominently in older individuals. Expression of JCHAIN was higher in plasmablasts from older individuals at baseline, in line with reported age-mediated increased activation/differentiation in the $\mathrm{B}$ cell compartment $^{49}$. Furthermore, gene signatures associated with commitment to plasma cells were more robust with mild disease. Interestingly, while mild disease was associated with accumulation and proliferation of marginal zone-like B cells, severe disease was associated with the accumulation of terminally differentiated memory B cells, which have been linked to adverse COVID-19 outcomes $^{46}$. Finally, transcriptional signatures associated with $\mathrm{B}$ cell activation and $\mathrm{NF}-\kappa \mathrm{B}$ signaling were dampened in older individuals with severe COVID-19. Coupled with lower levels of plasma CD40LG in older individuals with severe disease, these observations suggest a disruption in follicular helper $\mathrm{T}$ cell responses in older patients. While levels of binding antibodies did not vary with age, those of neutralization antibodies were significantly diminished in older individuals. Furthermore, older patients exhibited fewer Spike-specific 
a

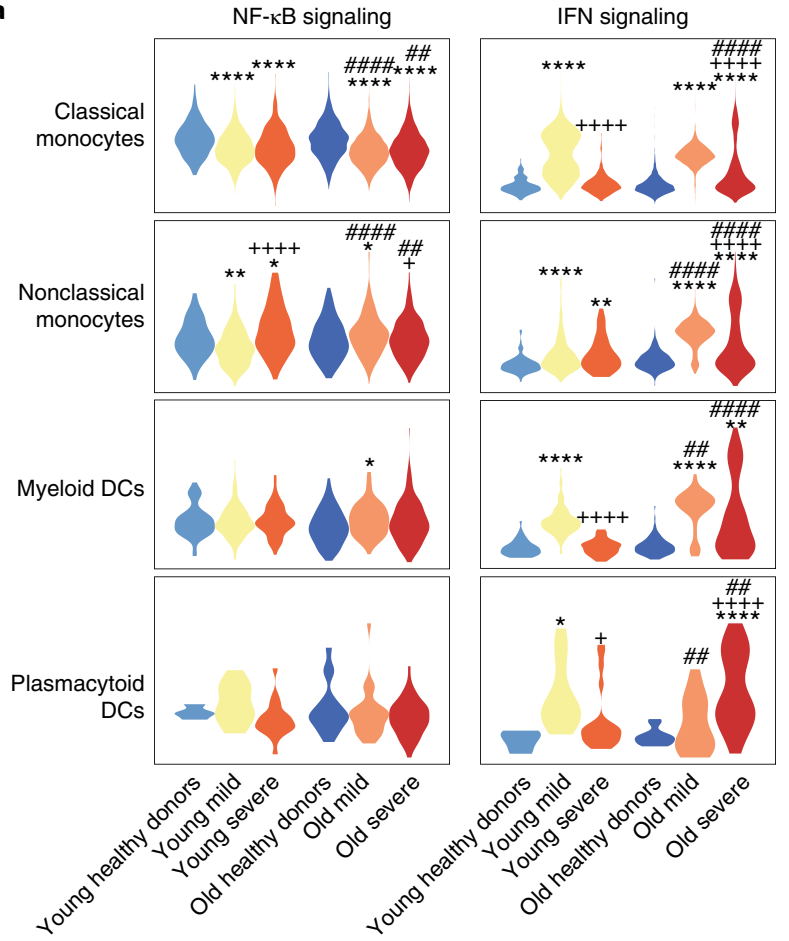

b

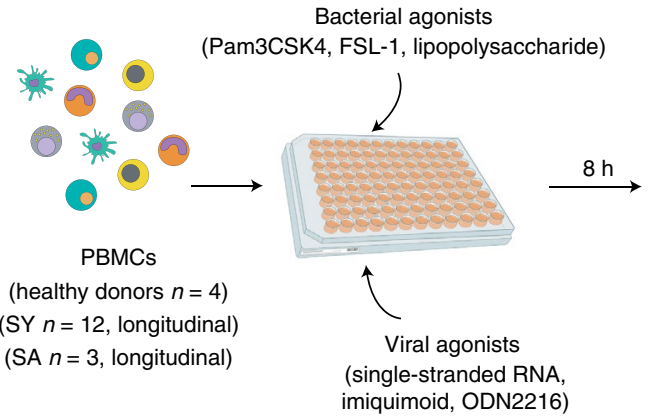

Intracellular staining (gated on monocytes, DCs)
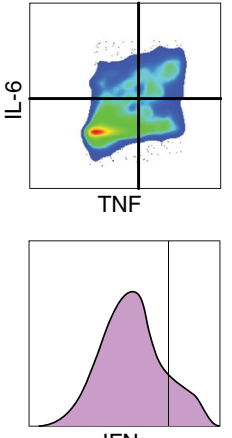
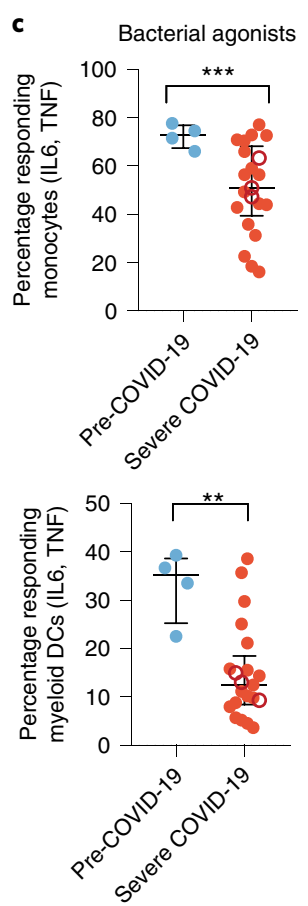

\section{d}
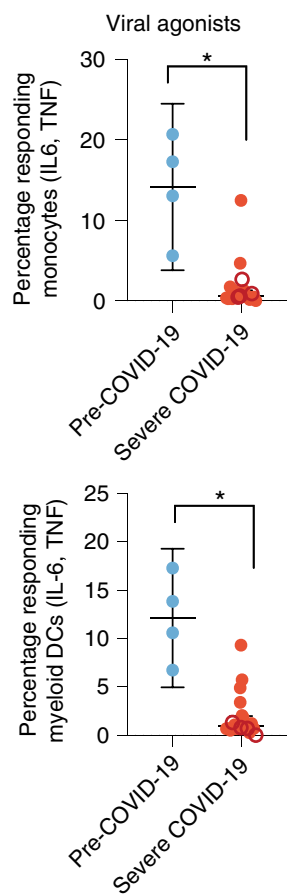
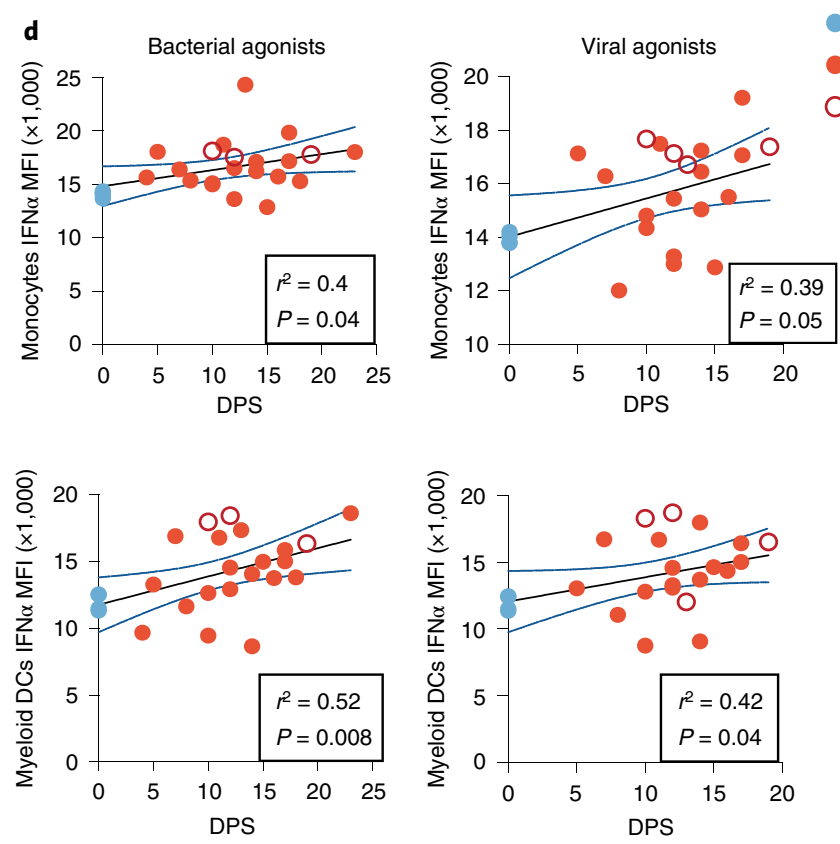

Fig. 7 | Dysregulation of TLR responses in myeloid cells from patients with severe COVID-19. a, Violin plots comparing IFN and NF- $K B$ signaling module scores in monocyte and DC subsets in patients with COVID-19 and healthy controls. b. Experimental design to measure the functional responses of monocytes and myeloid DCs from patients with severe COVID-19 and healthy donors created partially on Biorender.com. PBMCs collected longitudinally over the course of infection from young (SY) and aged (SA) patients with severe COVID-19 and healthy donors were stimulated with bacterial agonists (Pam3CSK4, lipopolysaccharide and FSL-1) and viral agonists (single-stranded RNA, imiquimod and ODN2216) separately for $8 \mathrm{~h}$. Cytokine responses (TNF, IL- 6 and IFN $\alpha$ ) were measured using intracellular staining followed by flow cytometry. c, Dot plots comparing the frequencies of monocytes (CD14+, top) and myeloid DCs (HLA-DR ${ }^{+} C D 11 c^{+}$, bottom) producing IL-6, TNF or both, after ex vivo stimulation with bacterial (left) and viral agonists (right) ( $n=4$ pre-COVID, 12 young severe, 3 old severe). d, Scatter plots with linear regression lines tracking the mean fluorescence of IFN $\alpha$ in IFN $\alpha^{+}$monocytes (top) and IFN $\alpha^{+}$myeloid DCs (bottom) after stimulation with bacterial (left) and viral agonists (right). The Spearman correlation and $P$ values are highlighted in each graph. Young patients with severe disease are shown by the filled circles whereas older patients with severe disease are shown by the empty circles. The $x$ axis denotes the DPS. The error bars represent the medians and IQR. Two-group comparisons were tested using a two-sided unpaired $t$-test with Welch's correction. Six-way comparisons were tested using a one-way ANOVA with Holm-Šídák multiple comparisons test where ${ }^{*}=$ significant compared to healthy donors, $+=$ significant compared to mild patients, $\#=$ significant difference between old mild/severe and young mild/severe groups. Two-way

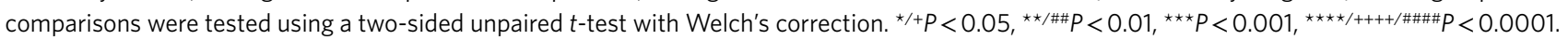


B cells. This observation agrees with recent studies demonstrating enrichment of ORF8-specific B cells in older adults, which are primarily nonneutralizing ${ }^{50}$. Collectively, these observations point at reduced plasticity of B cells with age or their increased reliance on CD4 T cell help, which have been shown to be impaired with severe COVID-19 in older individuals ${ }^{51,52}$.

$\mathrm{T}$ cell lymphopenia and impaired $\mathrm{T}$ cell responses are a hallmark of acute viral infection, including severe COVID-19 (refs. ${ }^{25,48,53,54}$ ). Our analyses revealed a significant drop in both total and naive $\mathrm{T}$ cells with DPS in older patients with severe COVID-19, notably within the CD8 $\mathrm{T}$ cell compartment. This drop could potentially be explained by increased trafficking to the site of infection or cytokine storm-induced apoptosis as reported for other viral diseases ${ }^{32}$. To that end, we observed a clear upregulation of cell death (Fas signaling) pathway in memory T cells in older patients. Moreover, severe COVID-19-associated metabolites in blood (such as lactic acid) can suppress $\mathrm{T}$ cell proliferation ${ }^{32}$. Indeed, our data highlight that while relatively abundant, $\mathrm{Ki} 67^{+}$proliferating $\mathrm{T}$ cells are reduced with age. These observations are in line with a progressive decrease in plasma levels of the T cell proliferation factors IL-2 and IL-7, and concomitant reduction in surface CD25 expression (IL2R) on CD8 T cells and IL-2R signaling module in older individuals. There is mounting evidence of $\mathrm{T}$ cell dysfunction/exhaustion with severe COVID-19 (refs. ${ }^{35,44,54,55}$ ). Gene expression differences in memory CD4 and CD8 T cells indicated increased T cell activation signatures with severe COVID-19 in the older patients. This hyperactivation of $\mathrm{T}$ cells could also explain the depletion of $\mathrm{T}$ cells, which is more significant in older patients. Functional exhaustion of T cells with severe COVID-19 is further evident from a lack of IFN $\gamma$ and IL-2 production in response to SARS-CoV-2 peptide pools containing open reading frames 7 and 8 . The poor antigenspecific responses coupled with reduced frequencies of activation markers (CD40L, CD69) support the conclusion that advanced age is associated with aberrant $\mathrm{T}$ cell activation potentially mediated by increased systemic inflammation and impaired $\mathrm{T}$ cell immunity in severe COVID-19 (ref. ${ }^{56}$ ).

The gene expression changes reported in this study also suggest that CD8 T cells from patients with severe disease exhibit increased cytotoxicity (based on increased expression of GZMB, PRF1, XCL1, $X C L 2)$ and NK cell markers (KLRD1), as previously reported using flow cytometry ${ }^{26}$. We also report an increase in the frequency of terminally differentiated, adaptive $\mathrm{CD}_{57}{ }^{+} \mathrm{NK}$ cells ${ }^{31}$, which were recently shown to be indicative of poor patient survival after severe disease $^{57}$. Functional exhaustion of NK cells has recently been described ${ }^{31,37,57}$ in the context of severe COVID-19. Our data suggest that this state is further exacerbated in older individuals, with lower frequencies of granzymeB ${ }^{+} \mathrm{NK}$ cells, circulating granzyme B levels and reduced expression of the IFNG and PRF genes in the more cytotoxic $\mathrm{CD}^{2} 6^{++} \mathrm{NK}$ cells in older individuals with severe disease.

Our data show an increase in nonclassical monocytes in both young and old patients with severe disease; however, an increased prevalence of classical monocytes is only evident in young individuals. In contrast to monocytes, a significant drop in both myeloid and plasmacytoid DCs in blood is observed with severe disease regardless of age ${ }^{30,46}$. As described recently $y^{28,30,58}$, we report a drop in MHC class II molecules (protein and RNA) on both monocytes and DC subsets independent of age, but HLA-DR expression recovers faster in young patients. This downregulation of HLA-DR in monocytes coupled with significantly lower CD86 expression suggests skewing of cells into a state of immune tolerance ${ }^{28}$. Indeed, our analysis within classical monocytes suggests a preferential trajectory of classical monocytes toward a regulatory cluster-expressing high levels of CD163 in patients with severe disease. Furthermore, monocytes and DCs from patients with severe COVID-19 exhibit attenuated response to Toll-like receptor (TLR) stimulation providing a potential explanation for increased susceptibility to secondary bacterial infections as described recently ${ }^{59,60}$. In contrast to the dampened response after stimulation with bacterial antigens, we report an elevated IFN signaling module with severe disease, particularly in myeloid DCs in older individuals. Stimulation of both monocytes and DCs with viral agonists targeting TLRs 7, 8 and 9 resulted in higher IFN production, suggesting innate immune signaling preferentially geared toward antiviral responses. Indeed, we detected a robust upregulation of the IFN signaling module across several cell types. Moreover, older individuals with severe disease exhibited stronger ISG signaling compared to young individuals, potentially due to delayed viral clearance. Exaggerated innate immunity in the lung, impaired antigen presentation and lymphopenia are hallmarks of other respiratory viral infections such as influenza and respiratory syncytial virus in the old ${ }^{18}$. Our analysis extends these observations and confirms the impairment of innate and adaptive immune responses in blood after severe COVID-19 in older patients.

We acknowledge several limitations in our study design and implementation. First, we analyzed immune parameters by DPS, which is self-reported and can be rather inaccurate and arbitrary. Second, we broadly defined patients with mild disease as ones with a positive PCR test with either no symptoms associated with COVID-19 or a mild disease not requiring an extensive ( $>3 \mathrm{~d})$ hospital stay. Lack of longitudinal samples from patients from this category prevented us from modeling disease dynamics with varied severity. Third, given the nature of this pandemic, there are some biases in patient and healthy donor cohorts. For example, healthy donors were predominantly female $(68 \%$ in the young; $58 \%$ in the old) while a significant number of patients with severe disease were Hispanic (69\% in the young; $75 \%$ in the old). Young patients with severe COVID-19 had elevated body mass index with respect to young healthy donors but there was no significance compared to the older patients with severe disease. Additionally, patients in the severe categories presented with a wide array of underlying conditions that might have played a role in disease severity/outcome. A significant number of patients were treated with remdesivir; however, there is limited evidence suggesting its role in either immune activation or suppression in the blood. Due to limited statistical power, we pooled patients with severe disease at any DPS time point into one category for initial analysis before regressing the data with time. Future studies will stratify young and old patients by clinical scores to identify innate immune correlates of disease severity and identify determinants of disease resolution and survival in patients with severe COVID-19. More importantly, it will address the impact of age/infection on qualitative differences in humoral responses and long-term durability of $\mathrm{T}$ cell responses to SARS-CoV-2 vaccine in the older population.

\section{Methods}

Ethics statement. This study was approved by the University of California, Irvine institutional review boards. Written informed consent was obtained from all enrolled participants.

Study participants and experimental design. Blood samples from patients admitted to the University of California, Irvine Medical Center (UCIMC) who participated in the National Institutes of Health (NIH) ACT-1 trial were used in these studies. Participants gave written consent to have the remainder of their blood samples used for ancillary studies related to COVID-19. Samples were stratified by disease severity--healthy donors, mild/moderate COVID-19 and severe COVID-19 and age ( $<60$ categorized as young and $\geq 60$ categorized as old where the median ages of young and old patients were 43 and 70 , respectively). Samples from healthy donors $(n=49 ; 37$ young and 12 old $)$ were obtained from previous unrelated studies collected before 2018 and blood from seronegative healthcare workers collected after March 2020. Individuals with asymptomatic/ mild disease ( $n=25 ; 13$ young and 12 old) were identified as those that tested positive for SARS-CoV-2 during their visit to UCIMC for reasons unrelated to COVID-19 symptoms (for example, heart attack, exacerbation of autoimmune disease, elective surgeries). These samples were obtained through the COVID-19 biospecimen bank at UCIMC. A total of 51 patients with severe COVID-19 ( 35 young and 16 old) were profiled, including patients with severe illness 
requiring hospitalization (ward), critical illness requiring ICU admission without/with intubation. For patients with severe disease, blood was collected longitudinally over several DPS, with the median DPS being 10 and $11 \mathrm{~d}$ for the young and old groups, respectively. Six patients (two young and four old) from our analysis succumbed to the disease. Due to limited sample availability, only a subset of samples were utilized in each of the assays. Detailed characteristics of the cohorts and experimental breakdown by sample are provided in Supplementary Table 1 .

Plasma and PBMC isolation. Whole-blood samples were collected in EDTA vacutainer tubes. PBMC and blood plasma samples were isolated after wholeblood centrifugation at $1,200 \mathrm{~g}$ for $10 \mathrm{~min}$ at room temperature in SepMate tubes (STEMCELL Technologies). Plasma was stored at $-80^{\circ} \mathrm{C}$ until analysis. PBMCs were cryopreserved using 10\% dimethyl sulfoxide/FCS in Mr. Frosty Freezing containers (Thermo Fisher Scientific) at $-80 \mathrm{C}$ then transferred to a cryogenic unit until analysis.

Measuring circulating immune mediators. The concentration of immune mediators in the plasma was measured using the Human XL Cytokine Discovery Premixed Kit (R\&D Systems) that includes cytokines (IFN $\alpha$, IFN $\beta$, IFN $\gamma$, IL- $1 \beta$, IL-10, IL-12p70, IL-13, IL-15, IL-17A, IL17E, IL-1RA, IL-2, IL-7, TNF, TRAIL, IL-33), chemokines (CCL2/MCP-1, CCL3/MIP-1 $\alpha$, CCL4/MIP-1 $\beta$, CCL20/MIP-3 $\alpha$, CCL5/RANTES, CCL11/eotaxin, CXCL1/GRO- $\alpha$, CXCL2/GRO- $\beta$, CXCL10/IP-10, CX3CL1/fractalkine), growth factors (GM-CSF, G-CSF, EGF, vascular endothelial growth factor, PDGF-AA, TGF $\alpha$ ) and effector molecules (granzyme B, PD-L1, CD40L, FLT3L). Samples were diluted according to the manufacturer's instructions and run in duplicate on the MAGPIX Instrument (Luminex). Data were fitted using a five parameter logistic regression on the xPONENT software v.7.0c.

Antibody end point titer ELISA. Clear 96-well, high-binding polystyrene ELISA plates were coated with $100 \mu \mathrm{l}$ per well of $500 \mathrm{ng} \mathrm{ml}^{-1}$ SARS-CoV-2 Spike protein receptor-binding domain (GenScript) in PBS overnight at $4^{\circ} \mathrm{C}$. Plates were blocked using $5 \%$ milk in wash buffer $(0.05 \%$ Tween in PBS) for $1 \mathrm{~h}$ at room temperature, washed three times with wash buffer and incubated with heat-inactivated $\left(55^{\circ} \mathrm{C}\right.$ for $30 \mathrm{~min}$ ) plasma samples (1:30 starting dilution) in threefold dilutions in duplicate for $1 \mathrm{~h}$. Responses were visualized by adding horseradish peroxidase (HRP)conjugated antihuman IgG (BD Pharmingen) or IgA (Cell Sciences) to the wells for another $90 \mathrm{~min}$ at room temperature, followed by three washes and the addition of o-phenylenediamine dihydrochloride substrate (Thermo Fisher Scientific) as substrate. The reaction was stopped using $1 \mathrm{M}$ of $\mathrm{HCl}$. Optical densities (ODs) were read at $490 \mathrm{~nm}$ on a VICTOR3 Multilabel plate reader (PerkinElmer). End point IgG and IgA titers were calculated using log transformation of the linear portion of the curve with 0.1 OD units as the cutoff. Titers were standardized using a positive-control sample included with each assay.

Focus reduction neutralization test. Heat-inactivated plasma serially diluted (1:3) in HyClone DMEM supplemented with $10 \mathrm{mM}$ of HEPES buffer were combined with SARS-CoV-2 (100 plaque-forming units) at $37^{\circ} \mathrm{C} 5 \% \mathrm{CO}_{2}$ for $1 \mathrm{~h}$. The antibody-virus inoculum was then transferred onto Vero E6 cells (C1008; ATCC) that were seeded in a 96 -well plate at $3.5 \times 10^{4}$ cells per well the previous day. After 1 -h incubation at $37^{\circ} \mathrm{C} 5 \% \mathrm{CO}_{2}, 1 \%$ methylcellulose (Sigma-Aldrich) in DMEM (5\% FCS) was overlaid on the infected Vero cell layer. Plates were incubated at $37^{\circ} \mathrm{C} 5 \% \mathrm{CO}_{2}$ for $24 \mathrm{~h}$. After $24 \mathrm{~h}$, the medium was carefully removed and the plates were fixed with $100 \mu \mathrm{l}$ of $10 \%$ neutral-buffered formalin for $1 \mathrm{~h}$ at room temperature. After fixation, plates were washed with deionized water and $50 \mu$ of ice-cold methanol supplemented with $0.3 \%$ of hydrogen peroxide was added to each well. Plates were incubated at $-20^{\circ} \mathrm{C}$ for $10 \mathrm{~min}$ followed by $20 \mathrm{~min}$ at room temperature. The plates were washed 3 times with deionized water and blocked for $1 \mathrm{~h}$ with $5 \%$ nonfat dry milk in PBS. Anti-SARS Nucleocapsid Protein Antibody (catalog no. NB100-56576; Novus Biologicals) at 1:1,000 dilution in 5\% nonfat dry milk/PBS was added. After an overnight incubation at $4{ }^{\circ} \mathrm{C}$, plates were washed 4 times with PBS, $40 \mu$ l of HRP-conjugated anti-rabbit IgG antibody (BioLegend) $1: 1,500$ was added to each well and plates were incubated for $2 \mathrm{~h}$ at room temperature. Plates were developed using TrueBlue HRP substrate and imaged on an EliSpot reader. Each plate included a positive and a negative control. The halfmaximal inhibitory concentration $\left(\mathrm{IC}_{50}\right)$ was calculated by nonlinear regression analysis using normalized counted foci on Prism 7 (GraphPad Software). One hundred percent of infectivity was obtained by normalizing the number of foci counted in the wells derived from the cells infected with SARS-CoV-2 virus in the absence of plasma.

\section{Phenotypic analysis of circulating lymphocytes and monocyte by flow}

cytometry. Frozen PBMCs were thawed, washed in FACS buffer (2\% FCS, 1 mM of EDTA in PBS) and counted on TC20 (Bio-Rad Laboratories) before surface staining using two independent flow panels (Extended Data Fig. 8). For the innate panel, the following antibodies were used: CD3 (clone SP34, 1:20 dilution; BD Pharmingen) and CD20 (clone 2H7, 1:20 dilution: BioLegend) for the exclusion of $\mathrm{T}$ and $\mathrm{B}$ lymphocytes, respectively. We further stained for CD56 (Brilliant Violet 711, 1:200 dilution; BioLegend), CD57 (HNK-1, 1:20 dilution; BioLegend), KLRG1 (clone
SA231A2, 1:20 dilution; BioLegend), CD16 (clone 3G8, 1:100 dilution; BioLegend), CD14 (clone M5E2, 1:100 dilution; BioLegend), HLA-DR (clone L243, 1:200 dilution; BioLegend), CD11c (clone 3.9, 1:20 dilution; Thermo Fisher Scientific), CD123 (clone 6H6, 1:20 dilution; BioLegend) and CD86 (clone IT2.2, 1:20 dilution; BioLegend). For the adaptive panel, the following antibodies were used: CD4 (clone OKT4, 1:20 dilution; BioLegend), CD8b (clone 2ST8.5H7, 1:100 dilution; Beckman Coulter), CD45RA (clone HI100, 1:20 dilution; Tonbo Biosciences), CCR7 (clone G043H7, 1:20 dilution; BD Biosciences), CD19 (clone HIB19, 1:200 dilution; BioLegend), IgD (clone IA6-2, 1:20 dilution; BioLegend), CD27 (clone M-T271, 1:20 dilution; BioLegend), KLRG1 (clone SA231A2, 1:20 dilution; BioLegend) and PD-1 (clone EH12.2H7, 1:20 dilution; BioLegend). Cells were surface-stained at $4^{\circ} \mathrm{C}$ for $30 \mathrm{~min}$, permeabilized, fixed and stained intracellularly with Ki67 (clone B56, 1:5 dilution; BD Biosciences) for the adaptive panel and granzyme B (clone QA16A02, 1:20 dilution; BioLegend) for the innate panel. Dead cells were excluded using the Ghost Dye Viability Dye (Tonbo Biosciences).

Monocytes from a subset of samples were phenotyped using an additional panel. Briefly cells were thawed, incubated with Fc blocking reagent (Human TruStain FcX; BioLegend) for $10 \mathrm{~min}$ at room temperature and stained at $4{ }^{\circ} \mathrm{C}$ for 30 min using the following panel: CD14 (clone M5E2, 1:100 dilution; BioLegend), HLA-DR (clone L243, 1:200 dilution; BioLegend), CD62L (clone DREG-56, 1:20 dilution; BioLegend), CD163 (clone GHI/61, 1:20 dilution; BioLegend), CCR5 (clone J418F1, 1:25 dilution; BioLegend), CD40 (clone 5C3, 1:20 dilution; BioLegend), CCR2 (clone FAB151C, 1:25 dilution; R\&D Systems), CD64 (clone 10.1, 1:100 dilution; BioLegend), CX3CR1 (clone 2A9-1, 1:20 dilution; BioLegend), CD80 (clone 2D10, 1:20 dilution; BioLegend), CD11b (clone ICRF44, 1:20 dilution; BioLegend) and monocyte blocker (Tru-Stain Monocyte Blocker; BioLegend). Samples were acquired on the Attune NxT acoustic focusing cytometer (Thermo Fisher Scientific).

$\mathrm{T}$ cells from a subset of samples were phenotyped using two separate panels. PBMCs were stained at $4^{\circ} \mathrm{C}$ for 30 min using either: panel 1, CD4 (clone OKT4, 1:20 dilution; BioLegend), CD8 (clone RPA-T8, 1:5 dilution; BD Biosciences), CD3 (clone OKT3, 1:20 dilution; BioLegend), CD38 (clone HIT2, 1:20 dilution; Tonbo Biosciences), HLA-DR (clone LN3, 1:20 dilution; BD Biosciences), PD-1 (clone EH12.2H7, 1:20 dilution; BioLegend), CD25 (IL-2R, clone BC96, 1:100 dilution; BioLegend), CD127 (IL-7R, clone RDR5, 1:20 dilution; Invitrogen), CD95 (clone DX2, 1:20 dilution; BioLegend), CTLA-4 (clone BNI3, 1:20 dilution; BioLegend); panel 2, CD4 (clone OKT4, 1:100 dilution; Tonbo Biosciences), CD3 (clone OKT3, 1:20 dilution; BioLegend), CD134 (OX40, clone BER-ACT35, 1:20 dilution; BioLegend), CD69 (clone FN50, 1:20 dilution; BioLegend), CD154 (CD40L, clone 24-31, 1:20 dilution; BioLegend), CD137 (4-1BB (4B4-1), 1:20 dilution; BioLegend) separately. Samples were washed and analyzed on the Attune NxT acoustic focusing cytometer. All flow data were analyzed using FlowJo v.10 (FlowJo LLC).

To detect antigen-specific B cells, approximately $1 \times 10^{6}$ PBMCs were stained with $100 \mathrm{ng}$ of full-length biotinylated Spike protein (Sino Biological) that was preincubated with streptavidin-Brilliant Violet 510 (BioLegend) at a 2:1 ratio for $1 \mathrm{~h}$ at $4{ }^{\circ} \mathrm{C}$ and CD20-fluorescein isothiocyanate (FITC) (clone 2H7, 1:20 dilution; BioLegend) for $30 \mathrm{~min}$ at $4^{\circ} \mathrm{C}$. Negative controls were PBMC-stained with streptavidin-phycoerythrin (BioLegend) and CD20-FITC. Samples were washed twice and resuspended in $200 \mu$ l FACS buffer before being analyzed on the Attune NxT acoustic focusing cytometer (Thermo Fisher Scientific).

Monocyte stimulation assay. Approximately $1 \times 10^{6}$ PBMCs were cultured in the presence of Roswell Park Memorial Institute 1640 medium (controls), bacterial and viral agonists at $37^{\circ} \mathrm{C}$. The bacterial agonist cocktail consisted of a combination of $2 \mu \mathrm{g} \mathrm{ml}^{-1}$ of Pam3CSK4 (TLR1/2 agonist; InvivoGen), $1 \mu \mathrm{g} \mathrm{ml}^{-1}$ of FSL-1 (TLR2/6 agonist; Sigma-Aldrich), and $1 \mu \mathrm{g} \mathrm{ml}$ of lipopolysaccharide (TLR4 agonist from Escherichia coli O111:B4; InvivoGen). The viral agonist cocktail consisted of $5 \mu \mathrm{g} \mathrm{ml}^{-1}$ of imiquimod (R848, TLR7 agonist; InvivoGen), $1 \mu \mathrm{g} \mathrm{ml}^{-1}$ of singlestranded RNA (ssRNA40/LyoVec, TLR8 agonist; InvivoGen) and $5 \mu \mathrm{g} \mathrm{ml}^{-1}$ of ODN 2216 (CpG ODN, TLR9 agonist; InvivoGen). Samples were stimulated for $1 \mathrm{~h}$ after which a protein transport inhibitor (brefeldin A) was added and incubated for an additional $7 \mathrm{~h}$ at $37^{\circ} \mathrm{C}$. Cells were then washed twice in FACS buffer and surfacestained using the following antibody cocktail: CD14 (clone M5E2, 1:100 dilution; BioLegend), HLA-DR (clone L243, 1:200 dilution; BioLegend) and CD11b (clone ICRF44, 1:20 dilution; BioLegend) for $30 \mathrm{~min}$ at $4{ }^{\circ} \mathrm{C}$. Stained cells were then fixed and permeabilized using fixation buffer (BioLegend) and incubated overnight with a cocktail of intracellular antibodies: IL-6 (clone MQ2-6A3, 1:5 dilution; BD Biosciences), TNF (clone MAb11, 1:100 dilution; BD Biosciences) and IFN $\alpha$ (clone LT27:295, 1:50 dilution; Miltenyi Biotec). Samples were acquired on the Attune NxT acoustic focusing cytometer (Thermo Fisher Scientific). Data were analyzed using FlowJo v.10.

T cell stimulation assay with SARS-CoV-2 overlapping peptide pools. T cells from healthy donors (young and old) and patients with severe disease (young and old) were magnetic-activated cell sorting-purified from PBMCs using anti-CD2 magnetic beads (Miltenyi Biotec). The purity of positive fractions was confirmed using flow cytometry and were $>90 \% ; 1 \times 10^{5} \mathrm{~T}$ cells per well were stimulated with $1 \mu \mathrm{g}$ of a SARS-CoV-2 overlapping peptide pool covering Nsp14/E/M/ORF3a/6/7a/7b/8/10 (15 amino acids overlapping by 12 amino 
acids; Thermo Fischer Scientific), anti-CD3/CD28 (positive control) or medium (negative control) in 96-well plates for $24 \mathrm{~h}$ at $37^{\circ} \mathrm{C}$ and $5 \% \mathrm{CO}_{2} . \mathrm{T}$ cell responses in convalescent patients served as a positive control. Plates were spun and supernatants was collected to measure IFN $\gamma$, IL-2, IL-10, TNF and IL-4 using a custom ProcartaPlex Human High Sensitivity Luminex kit (Invitrogen). Undiluted samples were run in duplicate on the MAGPIX Instrument. Data were fitted using a five parameter logistic regression on the xPONENT software.

scRNA library preparation. Cryopreserved PBMCs from each patient $(n=4$ per group for healthy donors and mild disease; $n=4$ per time point for severe disease) were thawed, washed and stained with $1 \mu \mathrm{g}$ per test cell-hashing antibody (TotalSeq B0251,B0254, B0256, B0260, clones LNH-95 and 2M2; BioLegend) for $30 \mathrm{~min}$ at $4^{\circ} \mathrm{C}$. Samples were washed three times in ice-cold PBS supplemented with 2\% FCS and sorted on the FACSAria Fusion (BD Biosciences) with Ghost Dye Red 710 viability dye (Tonbo Biosciences) for dead cell exclusion. Live cells were counted in triplicate on a TC20 Automated Cell Counter (Bio-Rad Laboratories) and pooled in groups of four based on disease severity and time point. Pooled cells were resuspended in ice-cold PBS with $0.04 \%$ bovine serum albumin in a final concentration of 1,200 cells $\mu^{-1}$. Single-cell suspensions were then immediately loaded on the 10X Genomics Chromium Controller with a loading target of 17,600 cells. Libraries were generated using the v.3.1 chemistry according to the manufacturer's instructions with additional steps for the amplification of hashtag oligo barcodes and library preparation using the Chromium Single Cell 3' Feature Barcoding Library Kit (10X Genomics). Libraries were sequenced on an Illumina NovaSeq with a sequencing target of 30,000 reads per cell RNA library and 2,000 reads per cell hashtag oligo barcode library.

scRNA-seq data analysis. Raw reads were aligned and quantified using the Cell Ranger Single-Cell Software Suite with Feature Barcode addition (v.4.0; 10X Genomics) against the GRCh38 human reference genome using the STAR aligner (v.2.7.9s). Downstream processing of aligned reads was performed using Seurat v.4.0. Droplets with ambient RNA (cells with fewer than 200 detected genes) and dying cells (cells with $>25 \%$ total mitochondrial gene expression) were excluded during initial quality control. Data objects from all groups were integrated using Seurat ${ }^{61}$ using the healthy, young donor samples as the reference. Data normalization and variance stabilization was performed on the integrated object using the SCTransform function where a regularized negative binomial regression was corrected for the differential effects of mitochondrial gene expression levels. The HTODemux function was then used to demultiplex donors and further identify doublets, which were then removed from the analysis. Dimension reduction was performed using RunPCA function to obtain the first 30 principal components followed by clustering using the FindClusters function in Seurat. Clusters were visualized using the UMAP algorithm as implemented by Seurat's runUMAP function. Cell types were assigned to individual clusters using the FindMarkers function with a fold-change cutoff of at least 0.4 and using a known catalog of well-characterized scRNA markers for human $\mathrm{PBMCs}^{62}$. Clusters from three major cell types (T and NK cells, myeloid cells, B cells) were further subsetted from the total cells and re-clustered to identify minor subsets within those groups. The list of cluster-specific markers identified from this study are cataloged in Supplementary Table 2. Figures were generated using Seurat, ggplot2 (v.3.3.1) and pheatmap (v.1.0.12).

Pseudo-temporal analysis. The pseudotime trajectory of CD4 and CD8 T cells and monocytes was reconstructed using Slingshot (v.1.2.0 ${ }^{63}$. The analysis was performed on each of these subsets individually with $\mathrm{T}_{\text {reg }}$ and mucosal-associated invariant $\mathrm{T}$ cells excluded from the $\mathrm{T}$ cell analysis due to unique developmental origins. For each set of cells, the UMAP dimensional reduction performed in Seurat was used as the input for Slingshot. For the calculation of the lineages and pseudotime in the T cell subsets, the naive clusters were set as the root state. In the monocytes, MS1 was set as the root state.

Differential expression analyses. Differential expression analysis was performed with MAST using the default settings in Seurat. All disease comparisons were performed relative to healthy donors from the corresponding age groups. Only statistically significant genes (fold-change cutoff $\geq 1.5$; adjusted $P \leq 0.05$ ) were included in the downstream analysis.

Module scoring and functional enrichment. For gene scoring analysis, we compared gene signatures and pathways from the Kyoto Encyclopedia of Genes and Genomes (https://www.genome.jp/kegg/pathway.html) (Supplementary Table 3) in subpopulations using Seurat's AddModuleScore function. CD8 T cell specific modules were derived from previously published work ${ }^{64}$. NK cell exhaustion scores were calculated using the aggregate expression of the exhaustion markers PDCD1 (PD-1), LAG3, HAVCR2 (TIM-3) and B3GAT1. Fas-Jnk signaling scores were calculated using the aggregate expression of FAS, FASLG, DAXX, MAP3K5, MAPK8, MAPK9 and MAPK10. HLA class II module scores were calculated using the aggregate expression of $H L A-D M(A, B), H L A-D P(A 1, B 1), H L A-D O$ $(A, B), H L A-D Q(A 1, A 2, B 1, B 2)$ and $H L A-D R(A, B 1, B 5)$. Overrepresentative gene ontologies were identified using one-, two-, four- and 8-way enrichment of differential signatures using Metascape (v.3.5) ${ }^{65}$. Functional enrichment networks were edited and annotated using Cytoscape v.3.6.1. All plots were generated using ggplot2 and Seurat. Differences in module scores were tested for statistical significance using a one-way analysis of variance (ANOVA).

Statistical analysis. Two-group comparisons were tested using a two-sided unpaired $t$-test followed with Welch's correction after testing for normality using a Shapiro-Wilk test with alpha levels set at 0.05 . Group differences were tested using an ordinary ANOVA test followed by Holm-Šídák multiple comparisons test with alpha levels set at 0.05 . Linear regression analysis compared significant shifts in curve over the horizontal line, with Spearman correlation coefficients reported for each age group. Group differences with regression were tested by fitting an analysis of covariance (ANCOVA) model on each parameter with age as a binary variable and DPS as a continuous covariate. Alpha levels were set at 0.1 .

Reporting Summary. Further information on research design is available in the Nature Research Reporting Summary linked to this article.

\section{Data availability}

The datasets supporting the conclusions of this article are available on the National Center for Biotechnology Information Sequence Read Archive under accession no. PRJNA727024. Further information and requests for resources should be directed to the corresponding author.

\section{Code availability}

The code used for the data analysis and figures in this study is available on GitHub (https://github.com/MessaoudiLab/COVID-19-Aging-Paper/).

Received: 3 February 2021; Accepted: 13 September 2021; Published online: 28 October 2021

\section{References}

1. Xie, J., Tong, Z., Guan, X., Du, B. \& Qiu, H. Clinical characteristics of patients who died of coronavirus disease 2019 in China. JAMA Netw. Open 3, e205619 (2020).

2. Nishiura, H. et al. Estimation of the asymptomatic ratio of novel coronavirus infections (COVID-19). Int. J. Infect. Dis. 94, 154-155 (2020).

3. Mizumoto, K. \& Chowell, G. Transmission potential of the novel coronavirus (COVID-19) onboard the Diamond Princess Cruises Ship, 2020. Infect. Dis. Model. 5, 264-270 (2020).

4. Nabors, C. et al. Characteristics and outcomes of patients 80 years and older hospitalized with coronavirus disease 2019 (COVID-19). Cardiol. Rev. 29, 39-42 (2021).

5. Grasselli, G. et al. Baseline characteristics and outcomes of 1591 patients infected with SARS-CoV-2 admitted to ICUs of the Lombardy region, Italy. IAMA 323, 1574-1581 (2020).

6. Mueller, A. L., McNamara, M. S. \& Sinclair, D. A. Why does COVID-19 disproportionately affect older people? Aging (Albany NY) 12, 9959-9981 (2020).

7. Levin, A. T. et al. Assessing the age specificity of infection fatality rates for COVID-19: systematic review, meta-analysis, and public policy implications. Eur. J. Epidemiol. 35, 1123-1138 (2020).

8. Chen, Y. et al. Aging in COVID-19: vulnerability, immunity and intervention. Ageing Res. Rev. 65, 101205 (2021).

9. $\mathrm{Li}$, J. et al. Clinical features of familial clustering in patients infected with 2019 novel coronavirus in Wuhan, China. Virus Res. 286, 198043 (2020).

10. Wang, D. et al. Clinical characteristics of 138 hospitalized patients with 2019 novel coronavirus-infected pneumonia in Wuhan, China. JAMA 323, 1061-1069 (2020).

11. Wu, J. et al. Clinical features of maintenance hemodialysis patients with 2019 novel coronavirus-infected pneumonia in Wuhan, China. Clin. J. Am. Soc. Nephrol. 15, 1139-1145 (2020).

12. Docherty, A. B. et al. Features of 20133 UK patients in hospital with Covid-19 using the ISARIC WHO Clinical Characterisation Protocol: prospective observational cohort study. BMJ 369, m1985 (2020).

13. Verity, R. et al. Estimates of the severity of coronavirus disease 2019: a model-based analysis. Lancet Infect. Dis. 20, 669-677 (2020).

14. Liu, K., Chen, Y., Lin, R. \& Han, K. Clinical features of COVID-19 in elderly patients: a comparison with young and middle-aged patients. J. Infect. 80, e14-e18 (2020).

15. Hazeldine, J. \& Lord, J. M. Immunesenescence: a predisposing risk factor for the development of COVID-19? Front. Immunol. 11, 573662 (2020).

16. Perrotta, F. et al. COVID-19 and the elderly: insights into pathogenesis and clinical decision-making. Aging Clin. Exp. Res. 32, 1599-1608 (2020).

17. Aiello, A. et al. Immunosenescence and its hallmarks: how to oppose aging strategically? A review of potential options for therapeutic intervention. Front. Immunol. 10, 2247 (2019)

18. Chen, J., Kelley, W. J. \& Goldstein, D. R. Role of aging and the immune response to respiratory viral infections: potential implications for COVID-19. J. Immunol. 205, 313-320 (2020). 
19. Akbar, A. N. \& Gilroy, D. W. Aging immunity may exacerbate COVID-19. Science 369, 256-257 (2020).

20. Blanco-Melo, D. et al. Imbalanced host response to SARS-CoV-2 drives development of COVID-19. Cell 181, 1036-1045.e9 (2020).

21. Laing, A. G. et al. A dynamic COVID-19 immune signature includes associations with poor prognosis. Nat. Med. 26, 1623-1635 (2020).

22. Lucas, C. et al. Longitudinal analyses reveal immunological misfiring in severe COVID-19. Nature 584, 463-469 (2020).

23. Vabret, N. et al. Immunology of COVID-19: current state of the science. Immunity 52, 910-941 (2020).

24. Del Valle, D. M. et al. An inflammatory cytokine signature predicts COVID-19 severity and survival. Nat. Med. 26, 1636-1643 (2020).

25. Giamarellos-Bourboulis, E. J. et al. Complex immune dysregulation in COVID-19 patients with severe respiratory failure. Cell Host Microbe 27, 992-1000.e3 (2020).

26. Kuri-Cervantes, L. et al. Comprehensive mapping of immune perturbations associated with severe COVID-19. Sci. Immunol. 5, eabd7114 (2020).

27. Hadjadj, J. et al. Impaired type I interferon activity and inflammatory responses in severe COVID-19 patients. Science 369, 718-724 (2020).

28. Schulte-Schrepping, J. et al. Severe COVID-19 is marked by a dysregulated myeloid cell compartment. Cell 182, 1419-1440.e23 (2020).

29. Osman, M. et al. Impaired natural killer cell counts and cytolytic activity in patients with severe COVID-19. Blood Adv. 4, 5035-5039 (2020).

30. Wilk, A. J. et al. A single-cell atlas of the peripheral immune response in patients with severe COVID-19. Nat. Med. 26, 1070-1076 (2020).

31. Maucourant, C. et al. Natural killer cell immunotypes related to COVID-19 disease severity. Sci. Immunol. 5, eabd6832 (2020).

32. Tan, L. et al. Lymphopenia predicts disease severity of COVID-19: a descriptive and predictive study. Signal Transduct. Target. Ther. 5, 33 (2020).

33. Chen, Z. \& John Wherry, E. T cell responses in patients with COVID-19. Nat. Rev. Immunol. 20, 529-536 (2020).

34. Zheng, M. et al. Functional exhaustion of antiviral lymphocytes in COVID-19 patients. Cell. Mol. Immunol. 17, 533-535 (2020).

35. Diao, B. et al. Reduction and functional exhaustion of $\mathrm{T}$ cells in patients with coronavirus disease 2019 (COVID-19). Front. Immunol. 11, 827 (2020).

36. Mazzoni, A. et al. Impaired immune cell cytotoxicity in severe COVID-19 is IL-6 dependent. J. Clin. Invest. 130, 4694-4703 (2020).

37. Westmeier, J. et al. Impaired cytotoxic $\mathrm{CD} 8^{+} \mathrm{T}$ cell response in elderly COVID-19 patients. mBio 11, e02243-20 (2020).

38. Zheng, Y. et al. A human circulating immune cell landscape in aging and COVID-19. Protein Cell 11, 740-770 (2020).

39. Caraux, A. et al. Phospholipase C- $\gamma 2$ is essential for NK cell cytotoxicity and innate immunity to malignant and virally infected cells. Blood 107, 994-1002 (2006).

40. Culley, F. J. et al. Role of CCL5 (RANTES) in viral lung disease. J. Virol. 80, 8151-8157 (2006).

41. Chen, Y. et al. IP-10 and MCP-1 as biomarkers associated with disease severity of COVID-19. Mol. Med. 26, 97 (2020)

42. Zhao, Y. et al. Longitudinal COVID-19 profiling associates IL-1RA and IL-10 with disease severity and RANTES with mild disease. JCI Insight 5, e139834 (2020).

43. Lang, F. M., Lee, K. M., Teijaro, J. R., Becher, B. \& Hamilton, J. A. GM-CSFbased treatments in COVID-19: reconciling opposing therapeutic approaches. Nat. Rev. Immunol. 20, 507-514 (2020).

44. De Biasi, S. et al. Marked T cell activation, senescence, exhaustion and skewing towards TH17 in patients with COVID-19 pneumonia. Nat. Commun. 11, 3434 (2020).

45. Pacha, O., Sallman, M. A. \& Evans, S. E. COVID-19: a case for inhibiting IL-17? Nat. Rev. Immunol. 20, 345-346 (2020).

46. Mann, E. R. et al. Longitudinal immune profiling reveals key myeloid signatures associated with COVID-19. Sci. Immunol. 5, eabd6197 (2020).

47. Bernardes, J. P. et al. Longitudinal multi-omics analyses identify responses of megakaryocytes, erythroid cells, and plasmablasts as hallmarks of severe COVID-19. Immunity 53, 1296-1314.e9 (2020).

48. Yu, K. et al. Dysregulated adaptive immune response contributes to severe COVID-19. Cell Res. 30, 814-816 (2020).

49. Castro, C. D. \& Flajnik, M. F. Putting J chain back on the map: how might its expression define plasma cell development? J. Immunol. 193, 3248-3255 (2014)

50. Dugan, H. L. et al. Profiling B cell immunodominance after SARS-CoV-2 infection reveals antibody evolution to non-neutralizingviral targets. Immunity 54, 1290-1303.e7 (2021).

51. Le Bert, N. et al. SARS-CoV-2-specific T cell immunity in cases of COVID-19 and SARS, and uninfected controls. Nature 584, 457-462 (2020).

52. Grifoni, A. et al. Targets of T cell responses to SARS-CoV-2 coronavirus in humans with COVID-19 disease and unexposed individuals. Cell 181, 1489-1501.e15 (2020).
53. Agrati, C. et al. Expansion of myeloid-derived suppressor cells in patients with severe coronavirus disease (COVID-19). Cell Death Differ. 27, 3196-3207 (2020).

54. Mathew, D. Deep immune profiling of COVID-19 patients reveals distinct immunotypes with therapeutic implications. Science 369, eabc8511 (2020).

55. Kalfaoglu, B., Almeida-Santos, J., Tye, C. A., Satou, Y. \& Ono, M. T-cell hyperactivation and paralysis in severe COVID-19 infection revealed by single-cell analysis. Front. Immunol. 11, 589380 (2020).

56. Rydyznski Moderbacher, C. et al. Antigen-specific adaptive immunity to SARS-CoV-2 in acute COVID-19 and associations with age and disease severity. Cell 183, 996-1012.e1019 (2020).

57. Varchetta, S. et al. Unique immunological profile in patients with COVID-19. Cell. Mol. Immunol. 18, 604-612 (2021).

58. Arunachalam, P. S. et al. Systems biological assessment of immunity to mild versus severe COVID-19 infection in humans. Science 369, 1210-1220 (2020).

59. Zhou, R. et al. Acute SARS-CoV-2 infection impairs dendritic cell and $\mathrm{T}$ cell responses. Immunity 53, 864-877.e5 (2020).

60. Ripa, M. Secondary infections in patients hospitalized with COVID-19: incidence and predictive factors. Clin. Microbiol. Infect. 27, 451-457 (2021).

61. Butler, A., Hoffman, P., Smibert, P., Papalexi, E. \& Satija, R. Integrating single-cell transcriptomic data across different conditions, technologies, and species. Nat. Biotechnol. 36, 411-420 (2018).

62. Zheng, G. X. et al. Massively parallel digital transcriptional profiling of single cells. Nat. Commun. 8, 14049 (2017)

63. Street, K. et al. Slingshot: cell lineage and pseudotime inference for single-cell transcriptomics. BMC Genomics 19, 477 (2018).

64. Singer, M. et al. A distinct gene module for dysfunction uncoupled from activation in tumor-infiltrating T cells. Cell 171, 1221-1223 (2017).

65. Zhou, Y. et al. Metascape provides a biologist-oriented resource for the analysis of systems-level datasets. Nat. Commun. 10, 1523 (2019).

\section{Acknowledgements}

We thank all participants in this study. We thank J. Atwood for assistance with sorting in the flow cytometry core at the Institute for Immunology, UCI. We thank M. Oakes from the UCI Genomics and High-Throughput Facility for assistance with 10X library preparation and sequencing. Aspects of the experimental design figures were generated using graphics from Biorender.com. We thank the support of the Chao Family Comprehensive Cancer Center resources, supported by the National Cancer Institute of the NIH under award no. P30CA062203. This study was supported by the National Center for Research Resources and the National Center for Advancing Translational Sciences, NIH, through grant nos. UL1 TR001414 (to D. Cooper), 1R01AI152258-01 (to I.M.), R21AI143301 (to I.M.) and 1R01AI145910-01 (to I.M.). S.A.L. is supported by NIH grant no. F31 A028704. N.S.R. is supported by NIH grant no. T32 AI007319. The content is solely the responsibility of the authors and does not necessarily represent the official views of the NIH.

\section{Author contributions}

S.A.L., S.S. and I.M. conceived and designed the experiments. D.T., F.Z., R.A.E., D. Chow, D. Cooper and A.A. facilitated procurement of the remnant blood samples from patients with COVID-19. S.A.L., S.S., M.Z.Z., B.D., M.C., I.C.I., A.J., A.N.P. and N.S.R. performed the experiments. S.A.L., S.S., M.Z., B.D., M.C. and N.S.R. analyzed the data. X.J. and W.S performed the statistical analyses. S.A.L., S.S. and I.M. wrote the paper. All authors have read and approved the final draft of the manuscript.

\section{Competing interests}

A.A. reports serving as principal investigator or coinvestigator of clinical trials sponsored by the NIH/National Institute of Allergy and Infectious Diseases, NeuroRx Pharma, Pulmotect, Blade Therapeutics, Novartis, Takeda, Humanigen, Eli Lilly, PTC Therapeutics, Octapharma, Fulcrum Therapeutics and Alexion. He has served as speaker or consultant for Bristol Myers Squibb, Pfizer, Boehringer Ingelheim, Portola, Sunovion, Mylan/Theravance, Salix, Alexion, AstraZeneca, Novartis, Nabriva, Paratek, Bayer, Tetraphase, Achaogen LaJolla, Millenium, Ferring, PeraHealth, HeartRite, AseptiScope and Sprightly. The other authors declare no competing interests.

\section{Additional information}

Extended data is available for this paper at https://doi.org/10.1038/s43587-021-00127-2.

Supplementary information The online version contains supplementary material available at https://doi.org/10.1038/s43587-021-00127-2.

Correspondence and requests for materials should be addressed to Ilhem Messaoudi.

Peer review information Nature Aging thanks Tracy Hussell, Janet Lord and the other, anonymous, reviewer(s) for their contribution to the peer review of this work.

Reprints and permissions information is available at www.nature.com/reprints.

Publisher's note Springer Nature remains neutral with regard to jurisdictional claims in published maps and institutional affiliations.

(c) The Author(s), under exclusive licence to Springer Nature America, Inc. 2021 

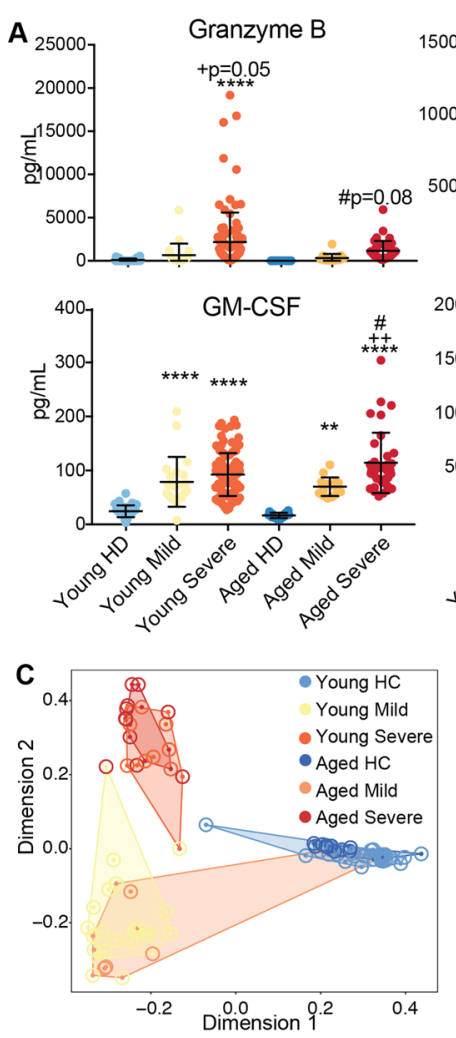
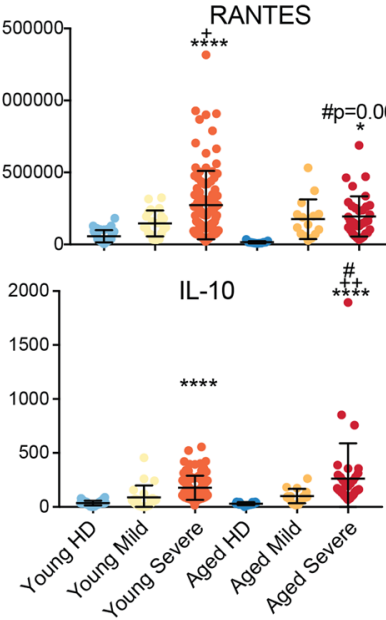

IP-10

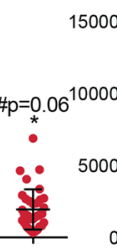

${ }^{200} 7$

D

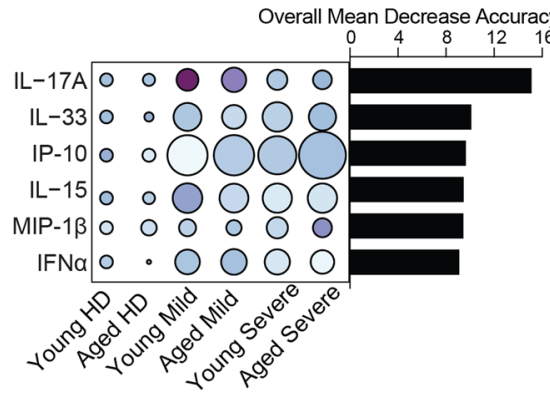

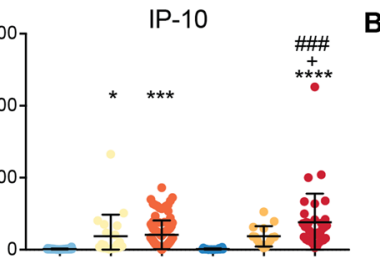

B

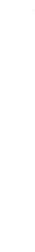

TNFa

\begin{tabular}{c|ccc} 
INFa & 0 & 0 & 0 \\
IL-7 & $\bigcirc$ & 0 & 0
\end{tabular}

IL-5 $\bigcirc 0$ 。

IL-4

$\mathrm{IL}-33$

IL-2

$\mathrm{IL}-1 \beta$

IL-1a

$\mathrm{IL}-13$

IL-12p70

MIP-1 $\beta$

CX3CL1

TGFa

PDGF-AA

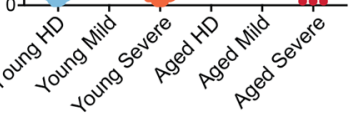

GRO- $\beta$

EGF

PD-L1

FLT-3L

CD40L vs. Young $\mathrm{HC}$

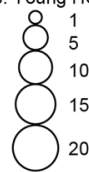

Within group Decrease in Accuracy

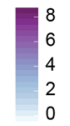

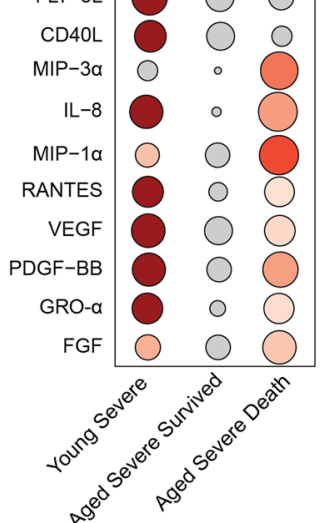

Extended Data Fig. 1 | Longitudinal profiling of soluble plasma mediators in mild and severe COVID-19 patients stratified by age. (a) Dot plots representing concentrations $(\mathrm{pg} / \mathrm{mL}$ ) of select cytokines, chemokines and growth factors measured by Luminex assay ( $n=21$ Young HD, 12 Aged HD, 9 Young Mild, 8 Aged Mild, 29 Young Severe, 11 Aged Severe). (b) Bubble plot representing linear regression analyses of the concentration (pg/mL) of select cytokines, chemokines, and growth factors across days post symptom onset (DPS). The size of the bubble represents positive correlation coefficient (spearman $r$ ) and the color represents the - $\log _{10}(\mathrm{p}$ value) significance of that positive correlation for each analyte in each of the three groups. (c) MDS plot of proximity scores from a random forest of circulating immune mediators at 1-5 DPS colored by age and disease severity. (d) Bubble plot of the top six most predictive variables (1-5 DPS) as ordered by mean decrease in accuracy. The size of the bubble represents the fold-change in concentration vs. young healthy controls and the color represents the decrease in accuracy for each group-mediator pair. Error bars represent medians and inter-quartile range. Six-way comparisons were tested using one-way ANOVA with Holm-Sidak's multiple test comparison where ${ }^{*}=$ significant compared to healthy donors, $+=$ significant compared to mild patients, \#=significant difference between Aged Mild/Severe and Young Mild/Severe groups. P-values: ${ }^{\star} /+/ \#=p<0.05$, ${ }^{\star *} /++=p<0.01,{ }^{* \star *} / \# \# \#=p<0.001,{ }^{* \star * *} /++++=p<0.0001$. 
A
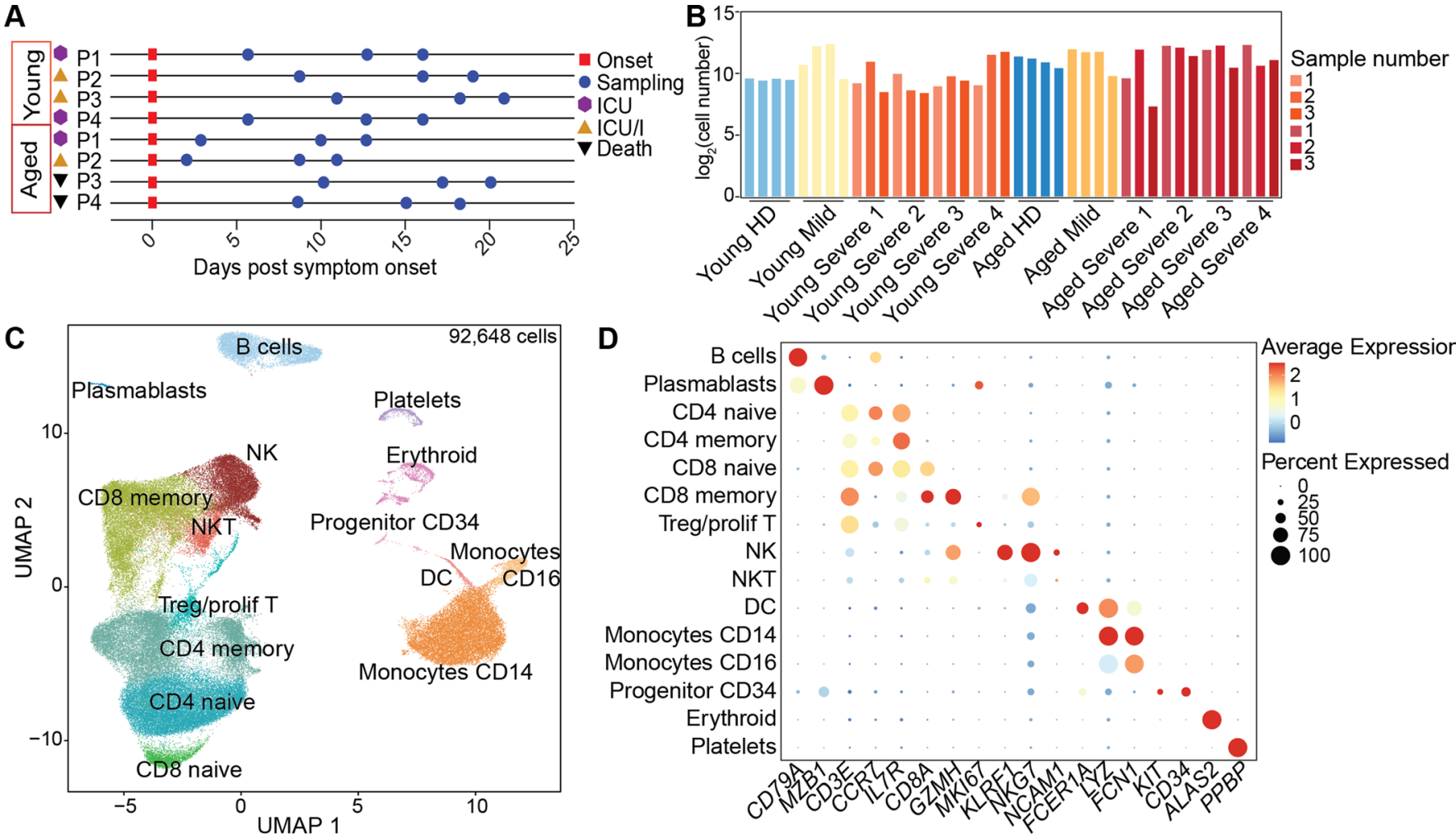

D

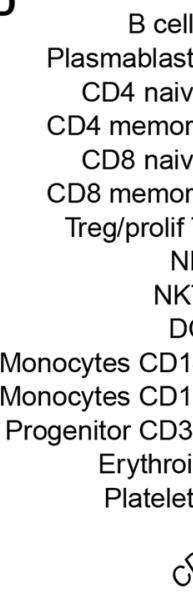

E
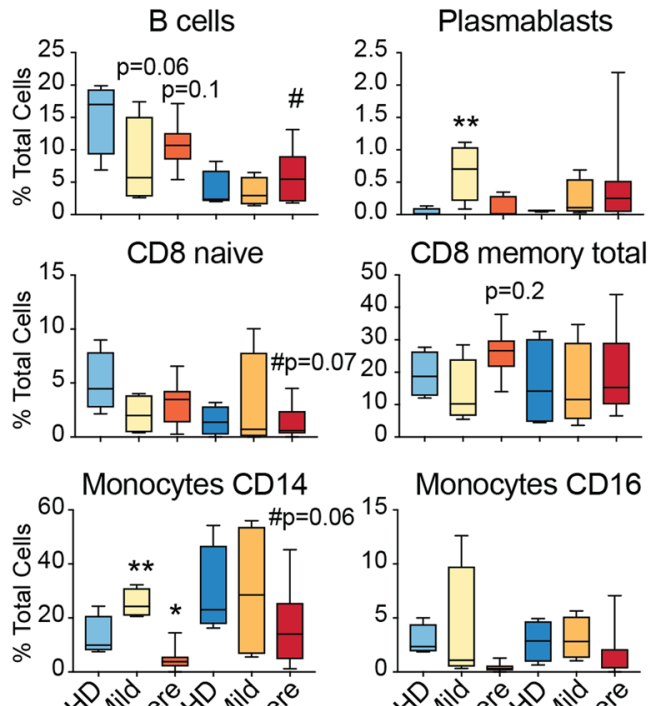

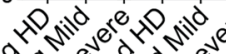
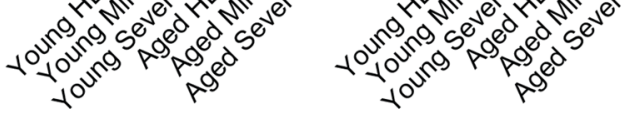
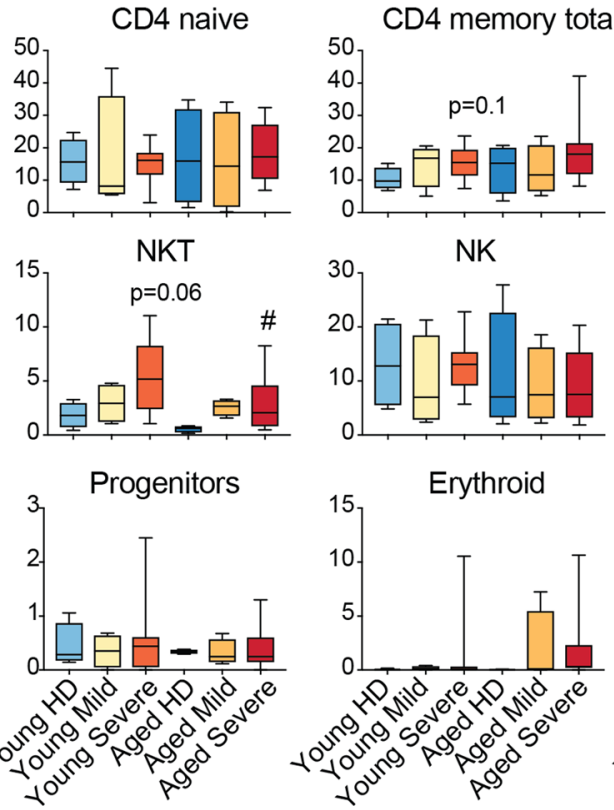

Average Expression

2

0

Percent Expressed

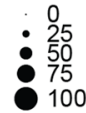

100

Extended Data Fig. 2 | Single cell RNA profiles of PBMC of mild and severe COVID-19 patients stratified by age. (a) Longitudinal sampling scheme for scRNA-seq from patients with severe COVID-19 with representative shape indicative of their clinical status/outcome. (b) Bar graph comparing the number of cells (log scale) captured from each patient at each timepoint by scRNA-Seq. (c) UMAP projection of all 92,648 cells isolated from both young and aged healthy donors, patients with mild and severe (longitudinally sampled) COVID-19. Major immune cell subsets are annotated. (d) Bubble plot of identifying genes highly expressed in each cluster determined using the FindMarkers function in Seurat. Level of normalized expression is shown using a color scale ranging from low (blue) to high (red). Size of the bubble is representative of the fraction of cells within each cluster expressing the marker. (e) Box plots comparing cell percentages calculated from clusters identified by dimensional reduction of all scRNA-Seq data $(n=4$ per group). The box hinges represent from $25^{\text {th }}$ to $75^{\text {th }}$ percentiles, the whiskers represent minimum and maximum values and the center line is the median. Six-way comparisons were tested using one-way ANOVA with Holm-Sidak's multiple test comparison where ${ }^{*}=$ significant compared to healthy donors, $+=$ significant compared to mild patients, $\#=$ significant difference between Aged Mild/Severe and Young Mild/Severe groups. P-values: ${ }^{\star} / \#=p<0.05,{ }^{\star \star}=p<0.01$. 
A

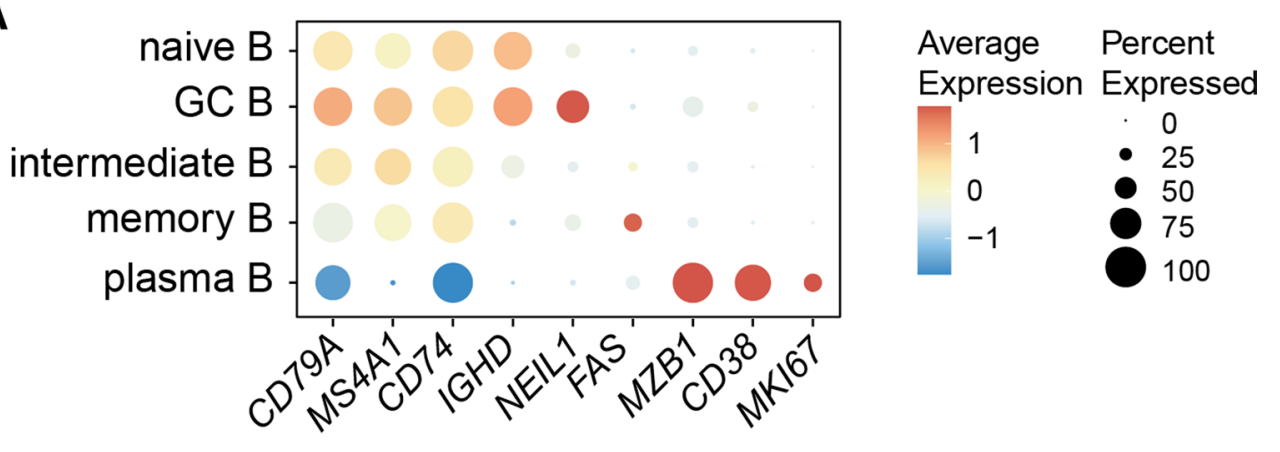

B End point titers (IgA)

C
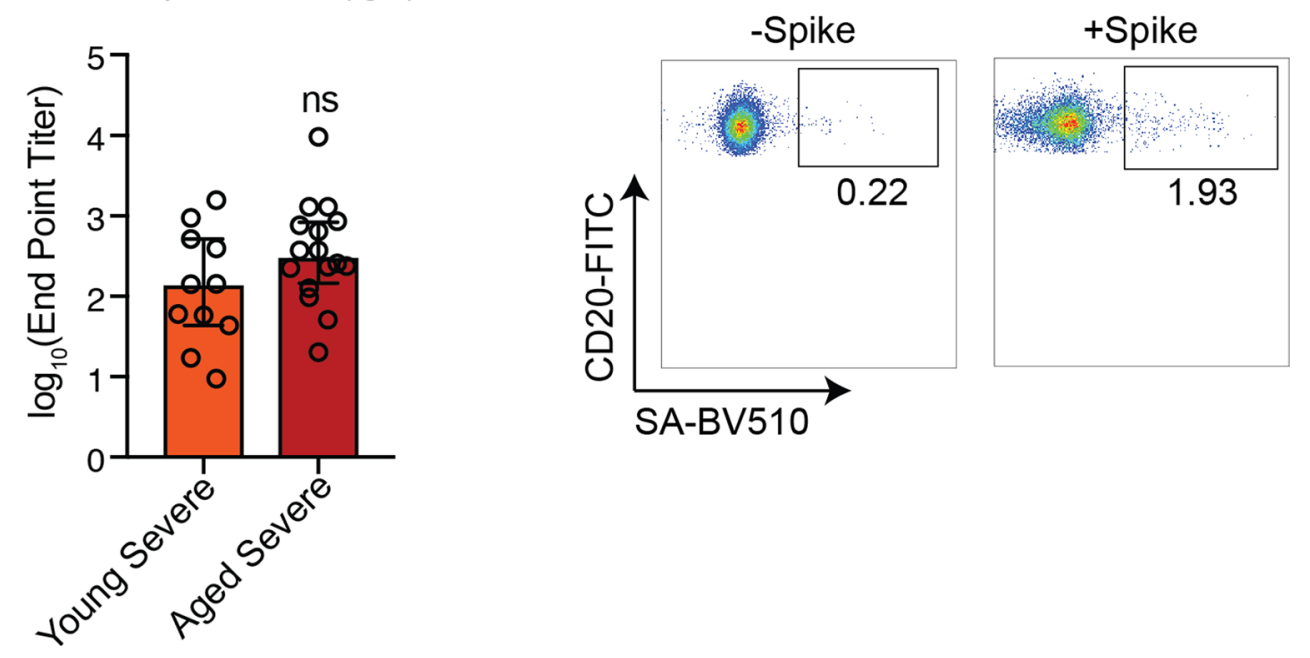

Extended Data Fig. 3 | Humoral responses to severe and mild COVD-19 in the young and aged. (a) Bubble plot of markers used to identify B cell clusters using Seurat's FindMarkers function. Normalized transcript levels are shown using a color scale ranging from low (blue) to high (red). Size of the bubble is representative of the fraction of cells within each cluster expressing the marker. (b) Bar plots of Spike-specific IgA End Point Titers from young and aged patients with severe COVID-19 ( $n=12$ Young from 7 individuals, $n=22$ Aged from 16 individuals). Error bars represent medians and inter-quartile range. Two-way comparison was tested using two-sided unpaired t-test with Welch's correction. (c) Representative flow plots for detection of antigen specific B cells in PBMC. 


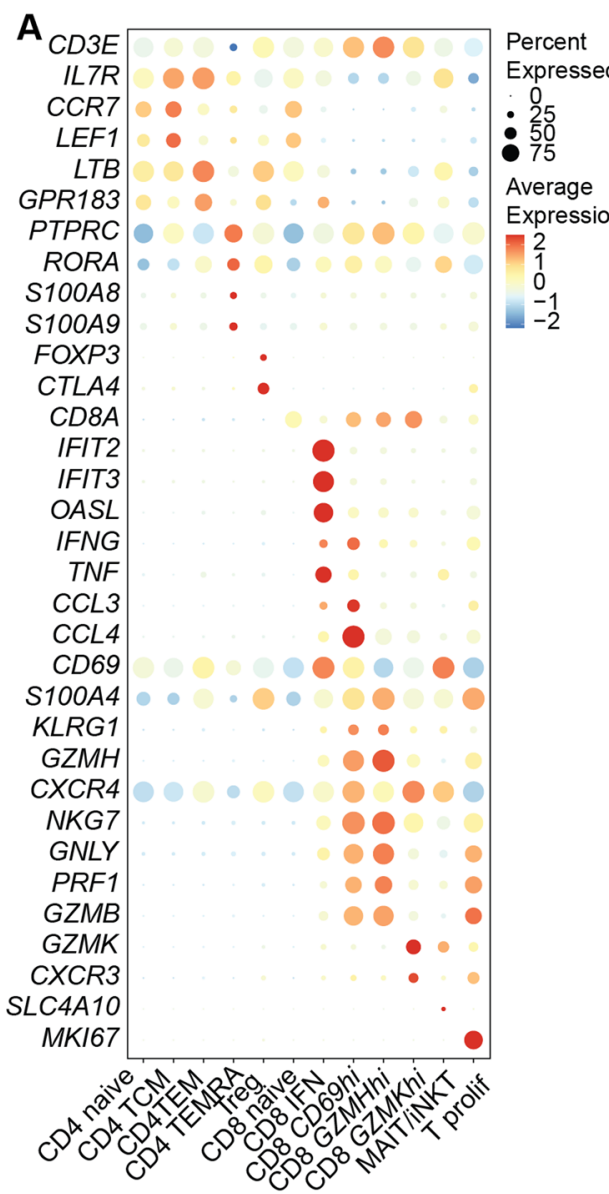

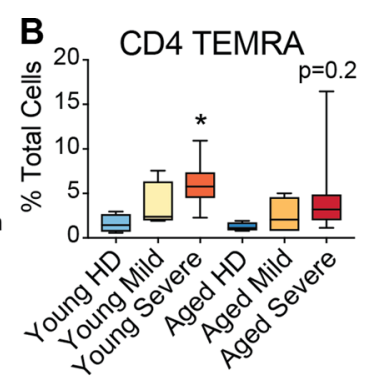
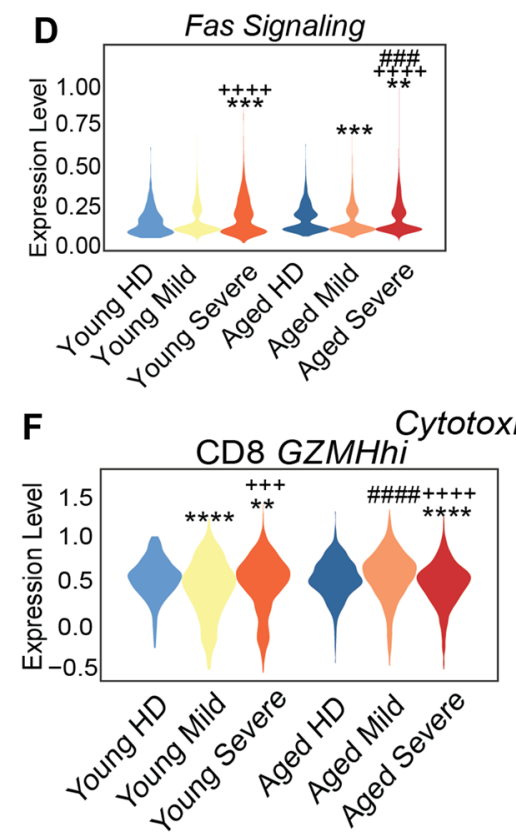

Downregulated in Severe Aged (253 DEG)

regulation of hemopoiesis $\quad 17$ pos. regulation of type I interferon production $\quad 8$

regulation of cellular response to stress $\quad 18$ negative regulation of cell cycle 19 response to peptide $\quad 17$ regulation of leukocyte differentiation $\quad 18$ regulation of viral transcription
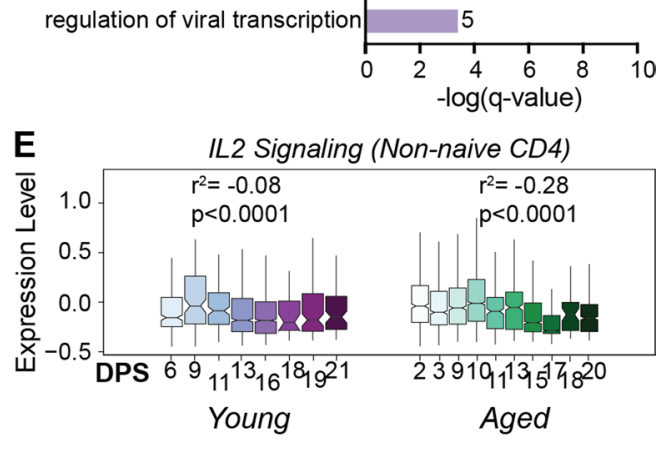
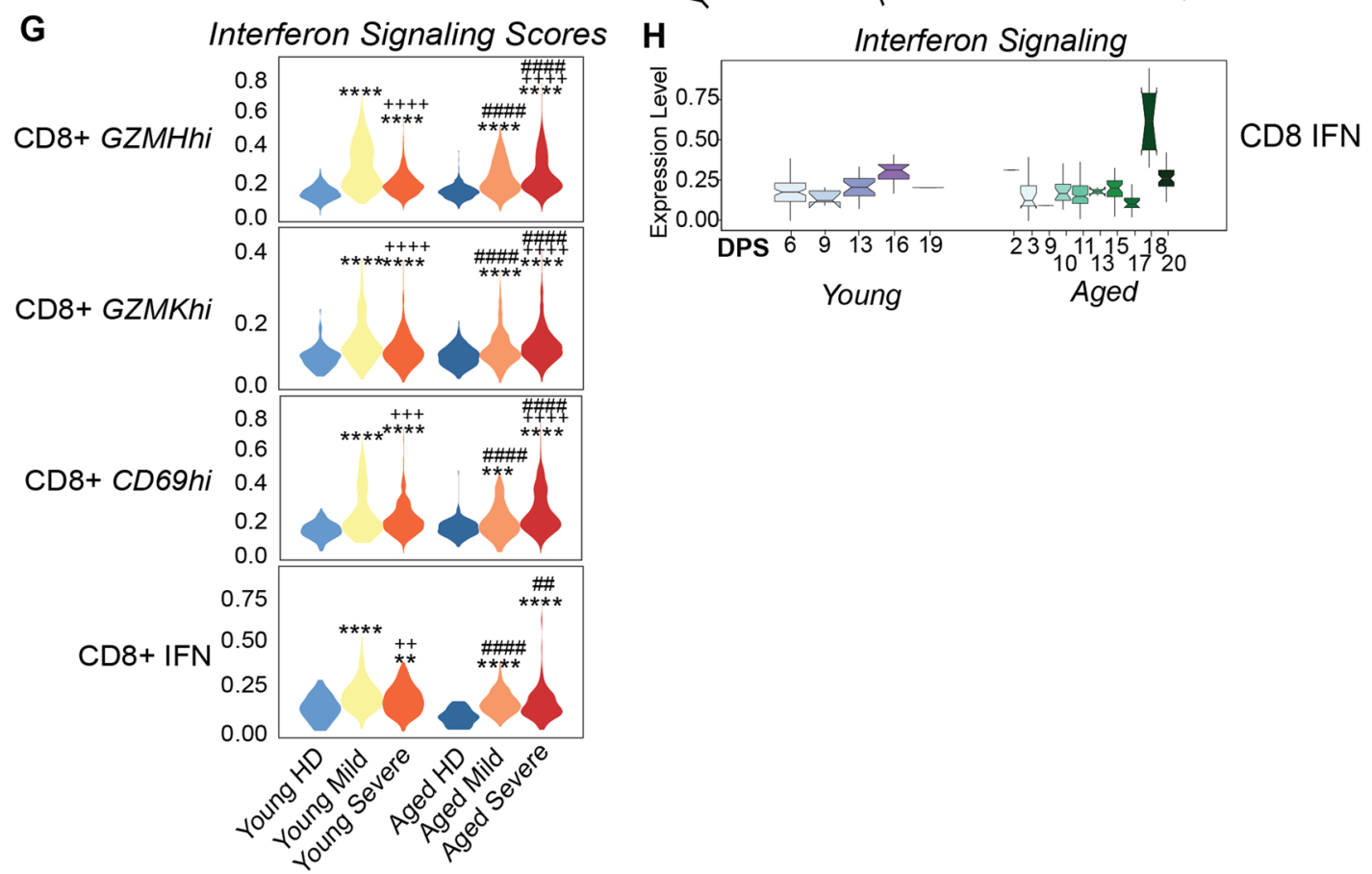

Extended Data Fig. 4 | See next page for caption. 
Extended Data Fig. 4 | Phenotypic and functional changes in T cell subsets with mild and severe COVID-19 in the elderly. (a) Bubble plot of genes highly expressed in each T cell cluster determined using the FindMarkers function in Seurat. Level of normalized expression is shown using a color scale ranging from low (blue) to high (red). Size of the bubble is representative of the fraction of cells within each cluster expressing the marker. (b) Box plots comparing frequencies of CD4 TEMRA cells within total cells from each patient identified from single cell analysis of T and NK cell subset ( $n=4 / g r o u p ;$ exact $p=0.032$ ). The box hinges represent from $25^{\text {th }}$ to $75^{\text {th }}$ percentiles, the whiskers represent minimum and maximum values and the center line is the median. (c) Bar graph showing over-representative GO terms in genes in CD4 TEMRA cells uniquely down-regulated with severe COVID-19 in aged subjects. Gene numbers associated with each term are highlighted next to each term. (d) Violin plot comparing Fas Signaling module scores within memory CD4 T cell clusters. (e) Box plots comparing longitudinal changes in IL-2 signaling module in memory CD4 T cell clusters from young and aged subjects with severe COVID-19. $X$ axis represents days post symptom onset (DPS). The box hinges represent from $25^{\text {th }}$ to $75^{\text {th }}$ percentiles, the whiskers represent minimum and maximum values and the center line is the median. (f) Violin plots comparing cytotoxicity module scores within GZMH ${ }^{\text {hi }}$ and $G Z M K^{\text {hi }} C D 8$ T cell clusters. (g) Violin plots comparing interferon signaling module scores within all memory CD8 T cell clusters. (h) Box plots comparing longitudinal changes in interferon modules in CD8 IFN T cells from young and aged subjects with severe COVID-19. X axis represents days post symptom onset (DPS). The box hinges represent from $25^{\text {th }}$ to $75^{\text {th }}$ percentiles, the whiskers represent minimum and maximum values and the center line is the median. Six-way comparisons were tested using one-way ANOVA with Holm-Sidak's multiple test comparison where ${ }^{*}=$ significant compared to healthy donors, $+=$ significant compared to mild patients, $\#=$ significant difference between Aged Mild/Severe and Young Mild/Severe groups. P-values: ${ }^{*}=p<0.05$,

$\star * /++/ \# \#=p<0.01,{ }^{\star \star \star} /+++/ \# \# \#=p<0.001,{ }^{* \star \star *} /++++/ \# \# \# \#=p<0.0001$. 
A

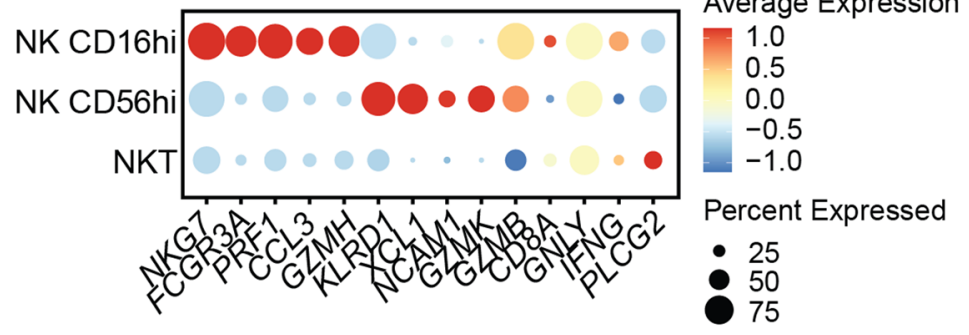

C

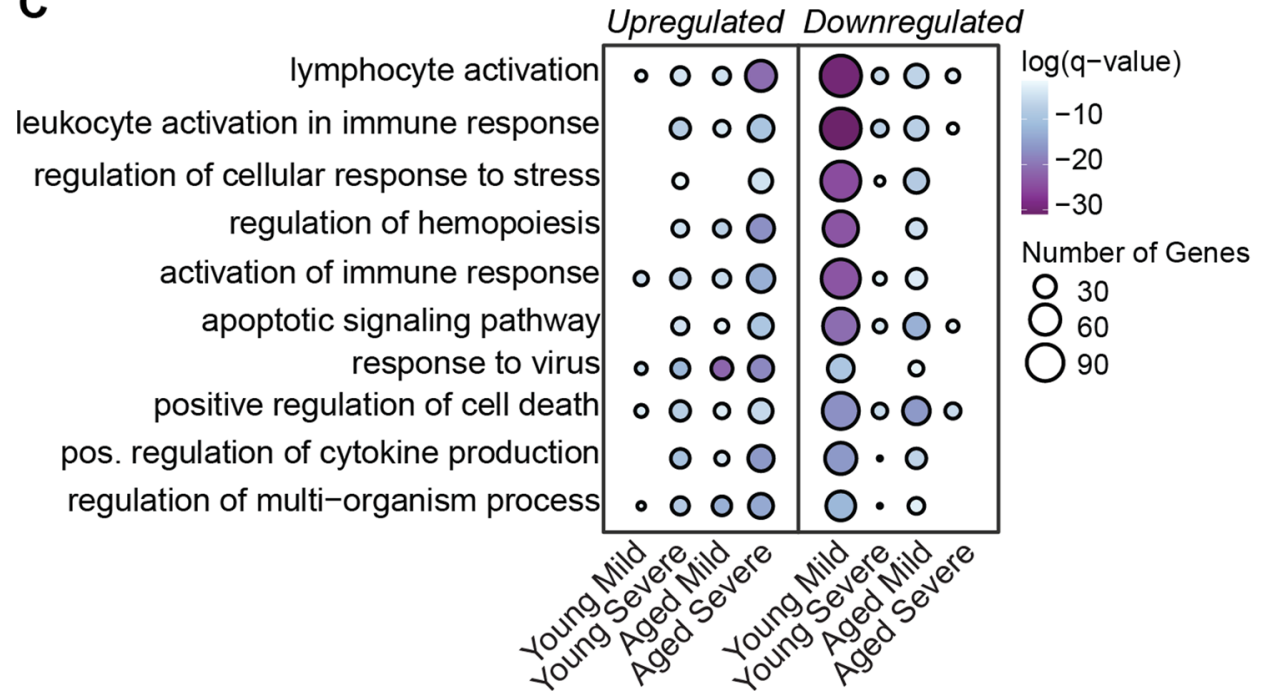

B

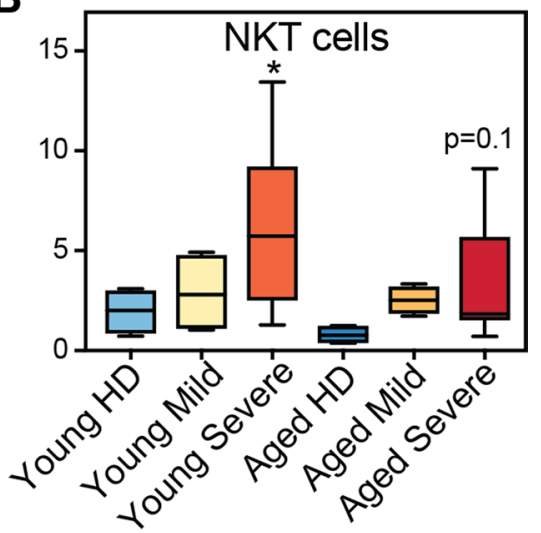

Extended Data Fig. 5 | Age-specific NK cell responses with mild and severe COVID-19. (a) Bubble plot of genes highly expressed in each NK cell cluster determined using the FindMarkers function in Seurat. Level of normalized expression is shown using a color scale ranging from low (blue) to high (red). Size of the bubble is representative of the fraction of cells within each cluster expressing the marker. (b) Box plots comparing frequencies of NKT cells (expressing PLCG2) within total cells in each patient identified from single cell analysis ( $n=4$ /age group/disease state; exact $p=0.041)$. The box hinges represent from $25^{\text {th }}$ to $75^{\text {th }}$ percentiles, the whiskers represent minimum and maximum values and the center line is the median. Six-way comparisons were tested using one-way ANOVA with Holm-Sidak's multiple test comparison where * =significant compared to healthy donors. (c) Bubble plot representing functional enrichment of DEG in CD16 high (FCGR3A $\left.{ }^{\text {high }}\right)$ NK cells from aged and young subjects with mild and severe COVID-19. Color and size of the bubble represents statistical significance and number of genes respectively. P-values: ${ }^{*}=p<0.05$. 
A

Classical Monocytes

B $\quad \mathrm{mDC}$

C
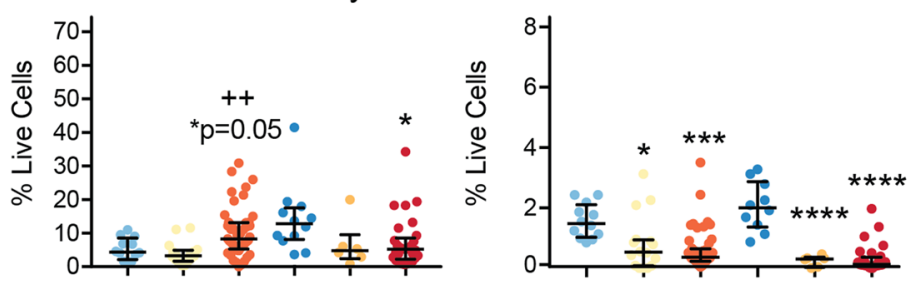

Monocytes
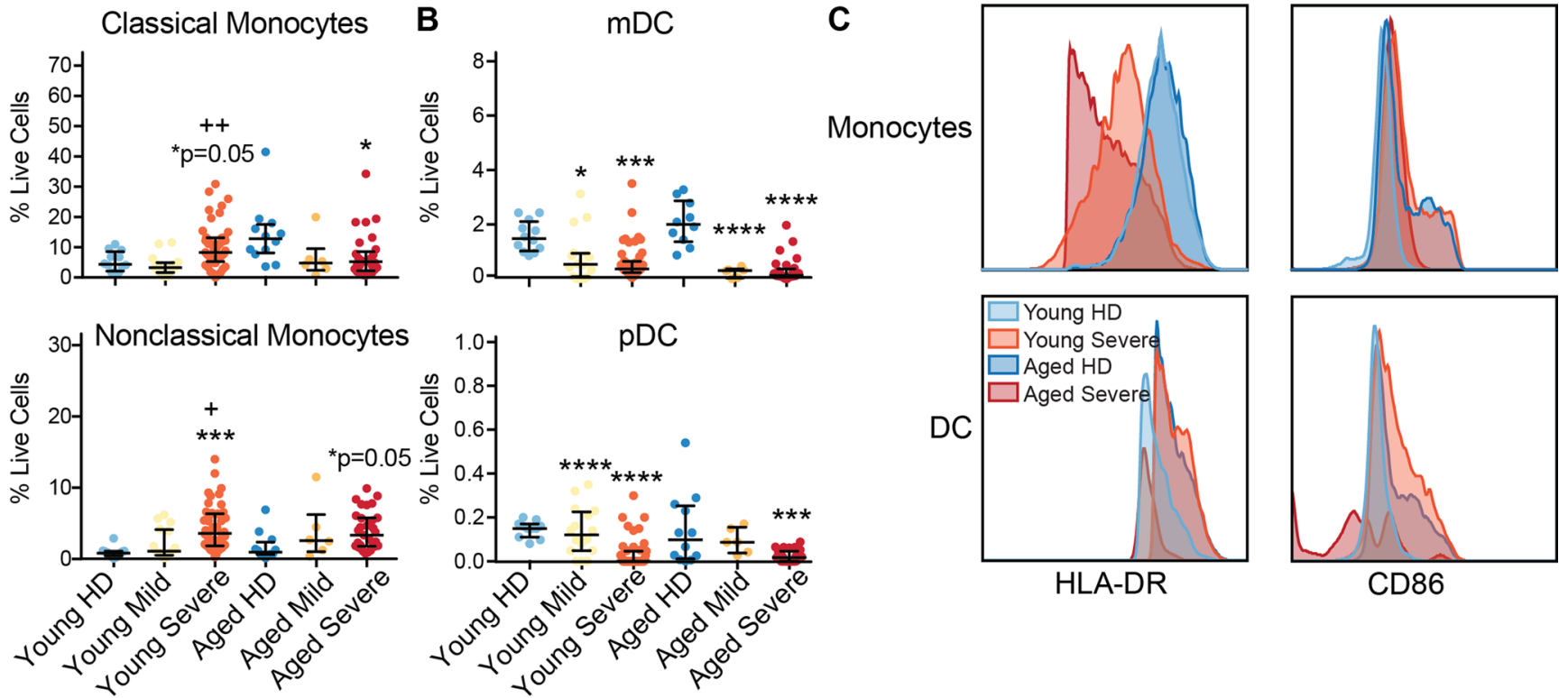

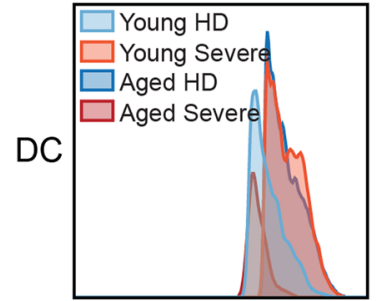

HLA-DR

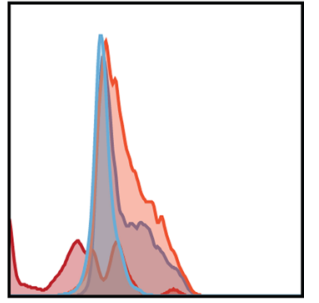

CD86

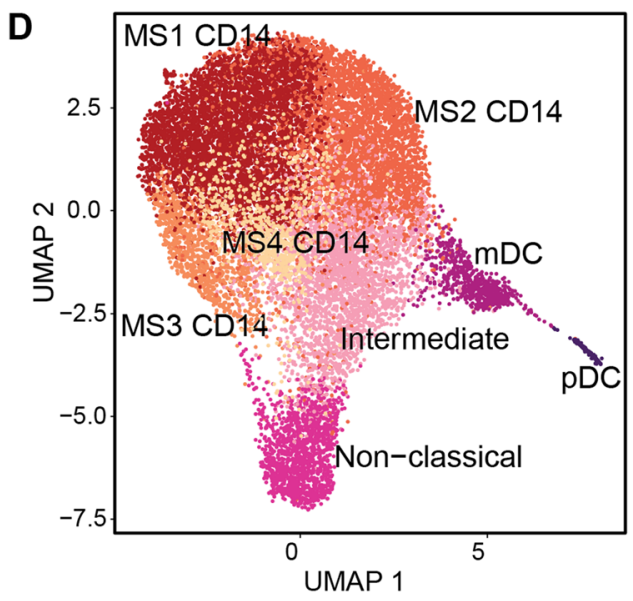

E

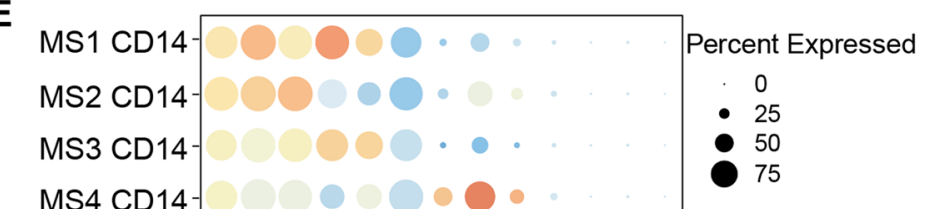

Intermediate- 000000 Average Expression

Non-classical

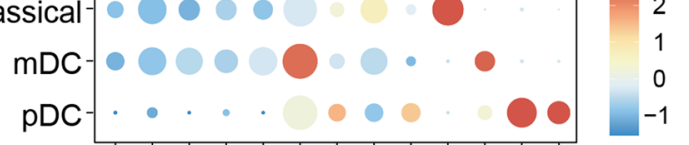

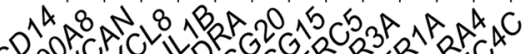

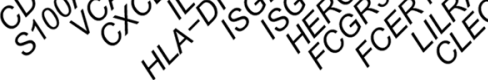
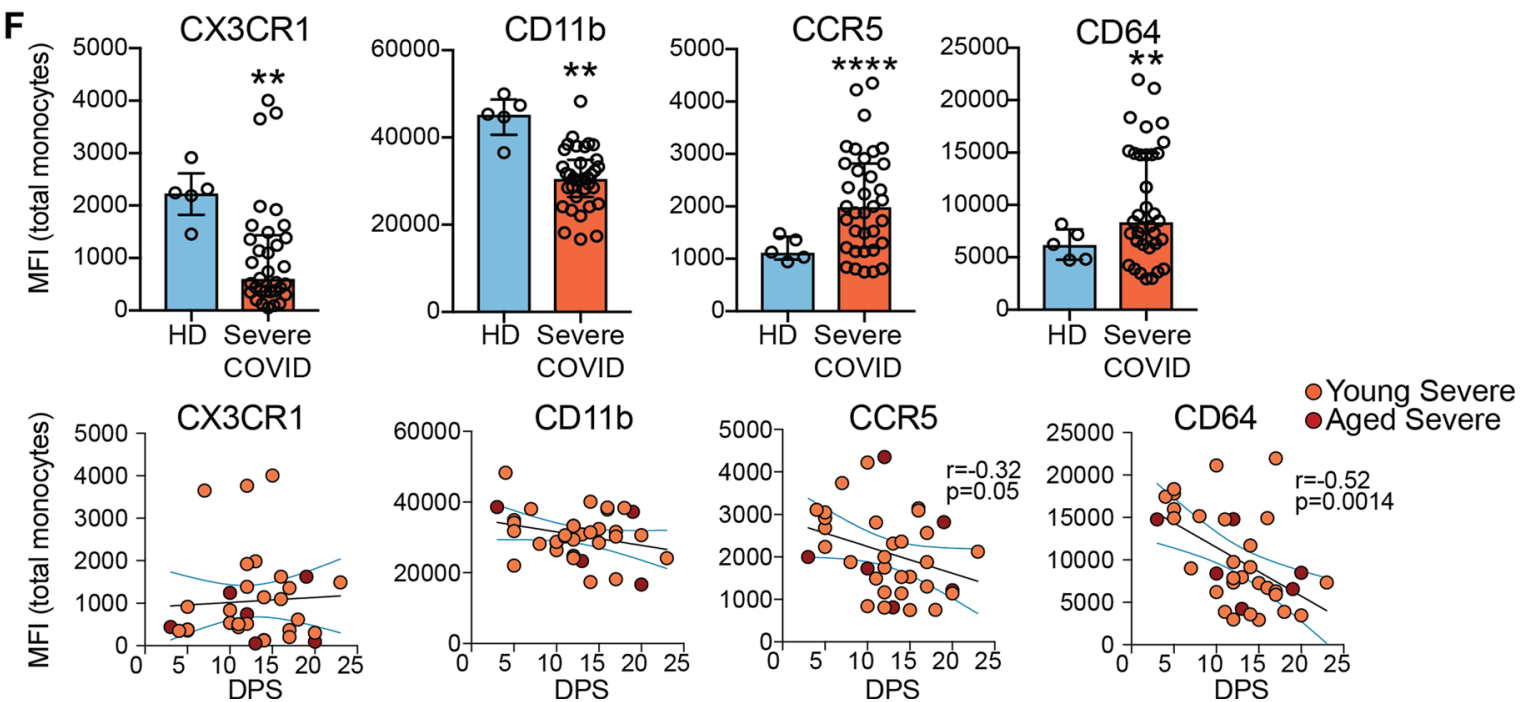

Extended Data Fig. 6 | See next page for caption. 
Extended Data Fig. 6 | Rewiring of myeloid cell phenotypes with severe COVID-19. (a-b) Dot plots comparing frequencies of (A) classical monocytes and non-classical monocytes as well as (b) myeloid DC (mDC) and plasmacytoid DC (pDC) using flow cytometry $(n=12$ Young HD, 12 Aged HD, 6 Young Mild, 4 Aged Mild, 27 Young Severe, 8 Aged Severe). (c) Histogram of surface expression of HLA-DR and CD86 expression from on total monocytes and DC. (d) UMAP projection of monocytes and DCs reclustered from the main UMAP. Major subsets of monocytes and DCs are highlighted. (e) Bubble plot of genes highly expressed in each cluster determined using Seurat's FindMarkers function. Level of normalized expression is shown using a color scale ranging from low (blue) to high (red). Size of the bubble is representative of the fraction of cells within each cluster expressing the marker. (f) Bar graphs comparing key monocyte surface markers in healthy donors (HD) and severe COVID-19 patients sampled during different stages of acute infection post symptom onset. Regression panel below each bar graph compares progression of the marker over the course of acute infection. Error bars represent medians and inter-quartile range. Six-way comparisons were tested using one-way ANOVA with Holm-Sidak's multiple test comparison where ${ }^{*}=$ significant compared to healthy donors, $+=$ significant compared to mild patients, \#=significant difference between Aged Mild/Severe and Young Mild/Severe groups. Two-way comparisons were tested using two-sided unpaired t-test with Welch's correction. P-values: ${ }^{\star} /+=p<0.05,{ }^{\star *} /++=p<0.01$, ${ }^{\star \star \star}=\mathrm{p}<0.001,{ }^{\star \star \star \star}=\mathrm{p}<0.0001$. 
A Young Aged

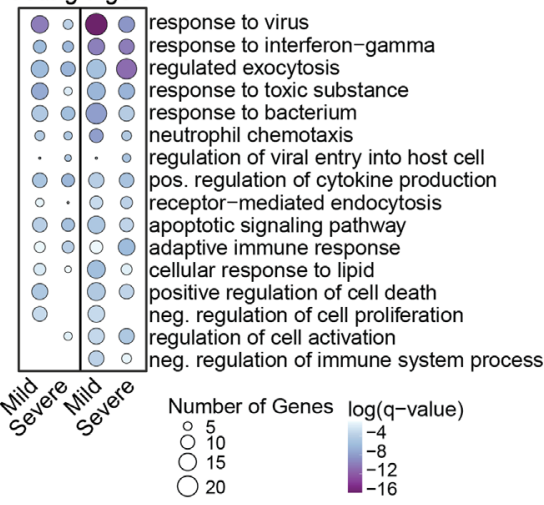

C

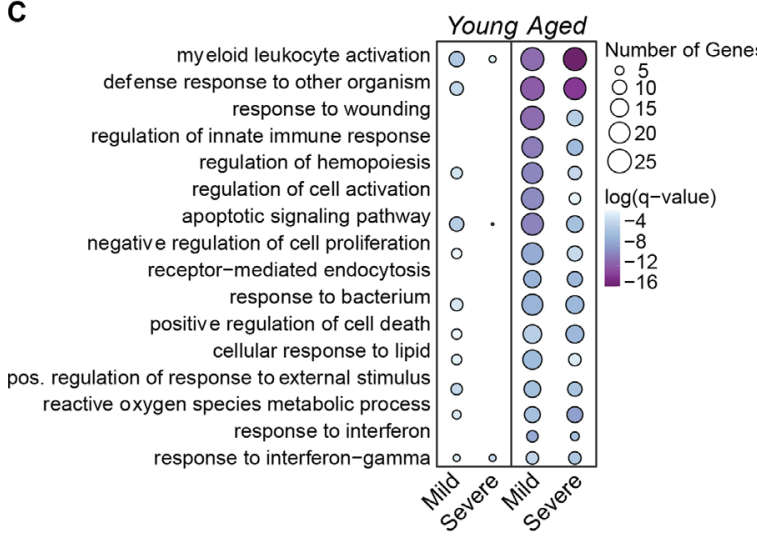

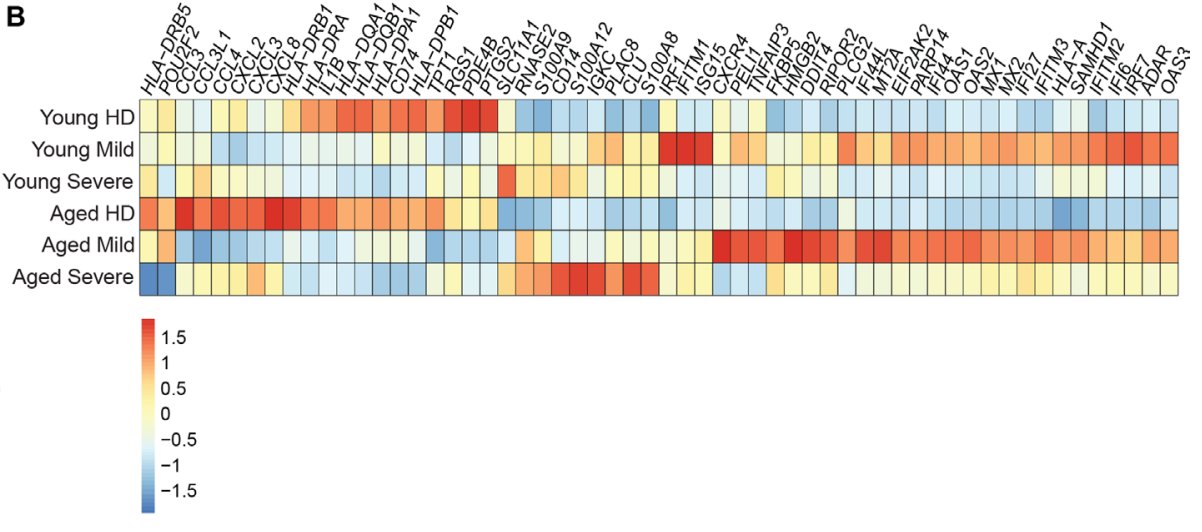

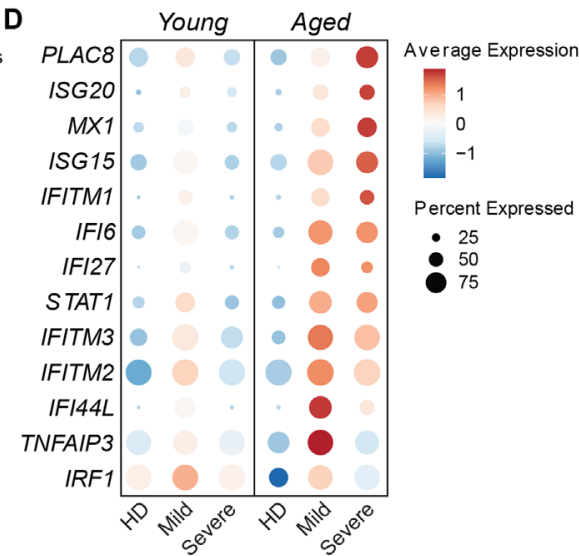

Extended Data Fig. 7 | Compromised monocyte responses to secondary insult with severe COVID-19. (a) Bubble plot of gene ontology terms derived from COVID-19 induced DEG in classical monocytes from aged and young subjects. Color and size of the bubble represents statistical significance and number of genes respectively. (b) Clustered heatmap comparing normalized transcript levels of genes enriching to GO terms 'response to virus' and 'response to interferon-gamma' within classical monocytes from young and aged patients with mild and severe COVID-19. Colors represent scaled gene expression ranging from blue (low) to red (high). (c) Functional enrichment of genes differentially expressed in non-classical monocytes following mild and severe COVID-19 Color and size of the bubble represents statistical significance and number of genes respectively. (d) Bubble plot showing gene expression changes in myeloid DCs from young and aged patients with mild and severe COVID-19. Size of the bubble is representative of the fraction of cells within each cluster expressing the marker. (e) Comparison of changes in RIG-I signaling module scores with COVID-19 in DC subsets. Sixway comparisons were tested using one-way ANOVA with Holm-Sidak's multiple test comparison where ${ }^{*}=$ significant compared to healthy donors, $\#=$ significant difference between Aged Mild/Severe and Young Mild/Severe groups. P-values: $\# \#=p<0.01, \# \# \#=p<0.001,{ }^{\star \star \star \star}=p<0.0001$. 

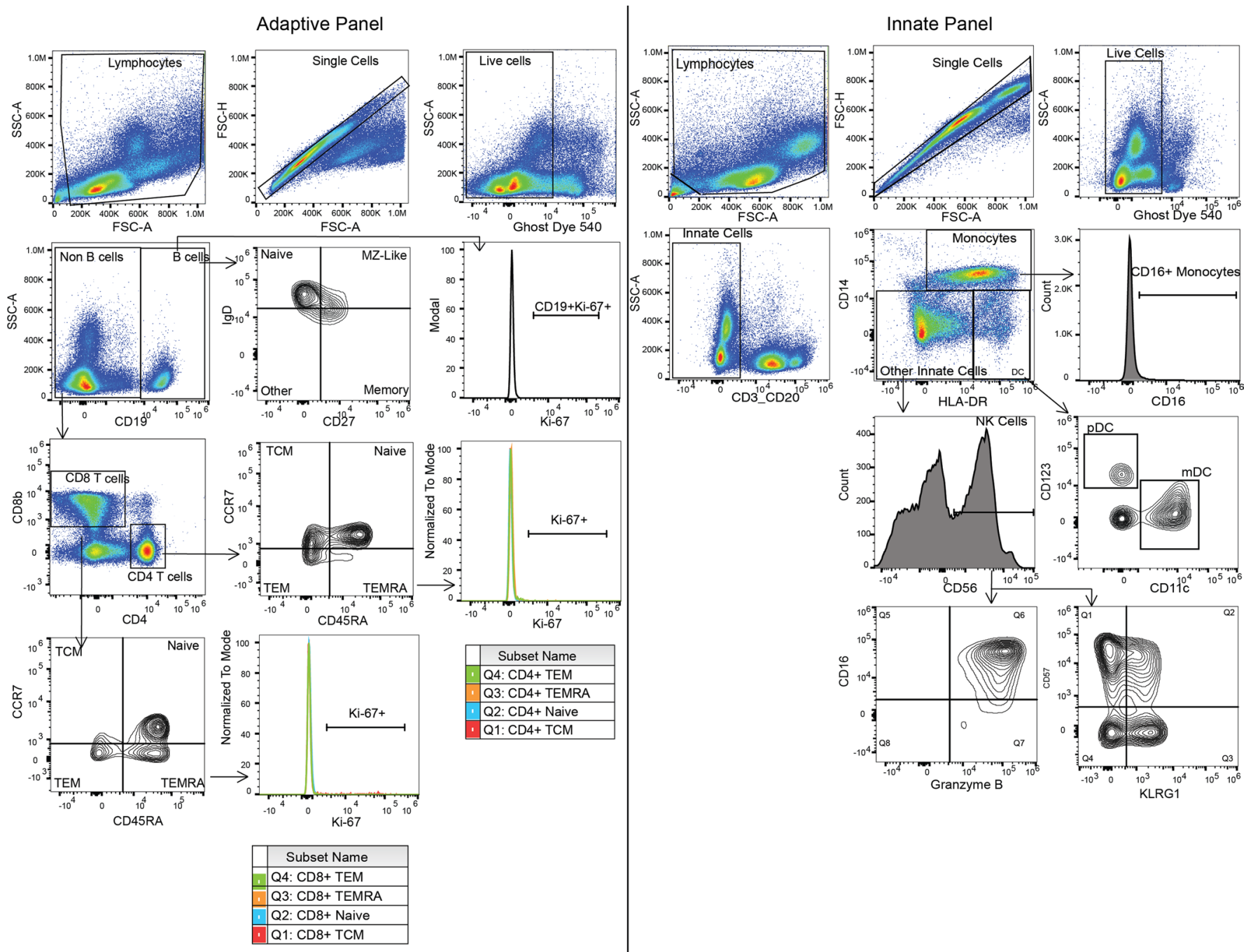

Extended Data Fig. 8 | Gating strategy for flow phenotyping. (a) Gating strategies for the adaptive and innate flow phenotyping panels. 


\section{Reporting Summary}

Nature Research wishes to improve the reproducibility of the work that we publish. This form provides structure for consistency and transparency in reporting. For further information on Nature Research policies, see our Editorial Policies and the Editorial Policy Checklist.

\section{Statistics}

For all statistical analyses, confirm that the following items are present in the figure legend, table legend, main text, or Methods section.

$\mathrm{n} / \mathrm{a}$ Confirmed

$\bigotimes$ The exact sample size $(n)$ for each experimental group/condition, given as a discrete number and unit of measurement

\ A statement on whether measurements were taken from distinct samples or whether the same sample was measured repeatedly

The statistical test(s) used AND whether they are one- or two-sided

Only common tests should be described solely by name; describe more complex techniques in the Methods section.

\A description of all covariates tested

$\square$ A description of any assumptions or corrections, such as tests of normality and adjustment for multiple comparisons

$\checkmark$ A full description of the statistical parameters including central tendency (e.g. means) or other basic estimates (e.g. regression coefficient)

AND variation (e.g. standard deviation) or associated estimates of uncertainty (e.g. confidence intervals)

For null hypothesis testing, the test statistic (e.g. $F, t, r$ ) with confidence intervals, effect sizes, degrees of freedom and $P$ value noted Give $P$ values as exact values whenever suitable.

Х $\square$ For Bayesian analysis, information on the choice of priors and Markov chain Monte Carlo settings

Х $\square$ For hierarchical and complex designs, identification of the appropriate level for tests and full reporting of outcomes

$\square \bigotimes$ Estimates of effect sizes (e.g. Cohen's $d$, Pearson's $r$ ), indicating how they were calculated

\section{Our web collection on statistics for biologists contains articles on many of the points above.}

\section{Software and code}

Policy information about availability of computer code

Data collection Sequencing data was collected using a NovaSeq S4. Attune NxT software for flow cytometry. Magpix instrument for Luminex assays using xPONENT software (version 7.0c).

Data analysis Raw reads were aligned and quantified using the Cell Ranger Single-Cell Software Suite with Feature Barcode addition (version 4.0, 10X Genomics) against the GRCh38 human reference genome using the STAR aligner.

R version 4.0.1 was used for downstream analysis with the following packages: Seurat (version 4.0), ggplot2 (3.3.1), sctransform (0.2.1), pheatmap (1.0.12), slingshot(1.2.0), and their package dependencies.

Flowjo 10.7.1 was used for analyzing flow cytometric data.

GraphPad Prism 7 was used for statistical analysis.

The code used for data analysis and figures in this study are available on Github https:/github.com/MessaoudiLab/COVID-19-Aging-Paper/.

For manuscripts utilizing custom algorithms or software that are central to the research but not yet described in published literature, software must be made available to editors and

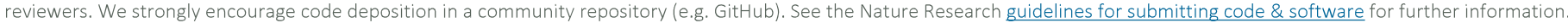

\section{Data}

Policy information about availability of data

All manuscripts must include a data availability statement. This statement should provide the following information, where applicable:

- Accession codes, unique identifiers, or web links for publicly available datasets

- A list of figures that have associated raw data

- A description of any restrictions on data availability

The datasets supporting the conclusions of this article are available on NCBI's Sequence Read Archive under the project "Differential dynamics of peripheral 


\title{
Field-specific reporting
}

Please select the one below that is the best fit for your research. If you are not sure, read the appropriate sections before making your selection.

$\bigotimes$ Life sciences $\quad \square$ Behavioural \& social sciences $\quad \square$ Ecological, evolutionary \& environmental sciences

For a reference copy of the document with all sections, see nature.com/documents/nr-reporting-summary-flat.pdf

\section{Life sciences study design}

All studies must disclose on these points even when the disclosure is negative.

Sample size $\quad 49$ healthy donors, 25 patients with mild/asymptomatic disease, and 53 patients with severe disease were obtained. Sample size was not predetermined. All available samples meeting criteria for the study were used in the assays described.

Data exclusions Droplets with ambient RNA (cells fewer than 200 detected genes) and dying cells (cells with more than $25 \%$ total mitochondrial gene expression) were excluded from scRNA-Seq data analysis. All exclusion criteria were pre-established for this data analysis.

Replication Due to the nature of sample retrieval and availability of viable sample, we were unable to perform technical replicates on individual samples.

Randomization Samples were not allocated into experimental groups.

Blinding Blinding to COVID-19 status or severity was not possible as patient and control samples were obtained from different locations and at different times.

\section{Reporting for specific materials, systems and methods}

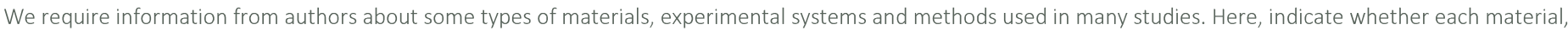

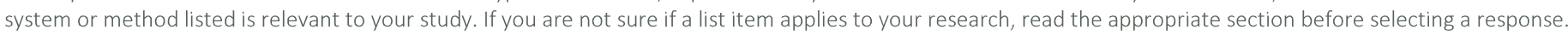

Materials \& experimental systems

$\mathrm{n} / \mathrm{a}$ Involved in the study

$\square$ \Antibodies

$\bigotimes \square$ Eukaryotic cell lines

Х $\square$ Palaeontology and archaeology

\ $\square$ Animals and other organisms

$\square$ \uman research participants

\ $\square$ Clinical data

\ $\square$ Dual use research of concern

\begin{tabular}{l|l}
\multicolumn{2}{l}{ Methods } \\
\hline n/a & Involved in the study \\
$\square$ & $\square$ ChIP-seq \\
$\square$ & $\bigotimes$ Flow cytometry \\
$\square$ & $\square$ MRI-based neuroimaging
\end{tabular}

\section{Antibodies}

Antibodies used

\begin{abstract}
CD3 (BD Cat.556611 Lot.9122674 Clone:SP34 Dil. Factor:20) CD20 (Biolegend Cat.302304 Lot.B218411 Clone:2H7 Dil. Factor:20) CD56 (Biolegend Cat.362542 Lot.b202209 Clone:5.1H11 Dil. Factor:200) CD57 (Biolegend Cat.359624 Lot.B313932 Clone:HNK-1 Dil. Factor:20) KLRG1 (Biolegend Cat.367716 Lot.B285217 Clone:SA231A2 Dil. Factor:20) CD16 (Biolegend Cat.302032 Lot.B309406 Clone:3G8 Dil. Factor:100) CD14 (Biolegend Cat.301822 Lot.b239383 Clone:M5E2 Dil. Factor:100) HLA-DR (Biolegend Cat.307618B Lot.b135067 Clone:L243 Dil. Factor:200) CD11c (Invitrogen Cat.61-0116-42 Lot.2270538 Clone:3.9 Dil. Factor:20) CD123 (Biolegend Cat.306016 Lot.b251504 Clone:6H6 Dil. Factor:20) CD86 (Biolegend Cat.305430 Lot.B312771 Clone:IT2.2 Dil. Factor:20) CD4 (BioLegend Cat.317440 Lot.B284698 Clone:OKT4 Dil. Factor:20) CD8b (Beckman Coulter Cat.6699512 Lot.CDS1447 Clone:2ST8.5H7 Dil. Factor:100) CD19 (Biolegend Cat.302208 Lot.B221392 Clone:HIB19 Dil. Factor:200) CCR7 (BD Cat.557648 Lot.100125 Clone:3D12 Dil. Factor:20) CD45RA (Tonbo Cat.65-0458-T100 Lot.C0458110320653 Clone:HI100 Dil. Factor:20) CD27 (Biolegend Cat.356430 Lot.B242545 Clone:M-T271 Dil. Factor:20) IgD (Biolegend Cat.348230 Lot.b205389 Clone:IA6-2 Dil. Factor:20) CD279(PD-1) (BioLegend Cat.329932 Lot.b220454 Clone:Eh12.2h7 Dil. Factor:20) Granzyme-B (Biolegend Cat.372208 Lot.B316858 Clone:QA16A02 Dil. Factor:20)
\end{abstract}


Ki67 (BD Cat.5136524x Lot.6077735 Clone:B56 Dil. Factor:5)

CD62L (Biolegend Cat.304805 Lot.B261323 Clone:DREG-56 Dil. Factor:20)

CD163 Biolegend Cat.333-608 Lot.b234324 Clone:GHI/61 Dil. Factor:20

CCR5 Biolegend Cat.359124 Lot.B187701 Clone:J418F1 Dil. Factor:25

CD40 BioLegend Cat.334336 Lot.b273300 Clone:5C3 Dil. Factor:20

CCR2 R\&D Cat.Fab151C Lot.AAQ00616101 Clone:48507 Dil. Factor:25

CD64 Biolegend Cat.305042 Lot.B276501 Clone:10.1 Dil. Factor:100

CX3CR1 Biolegend Cat.341604 Lot.b208844 Clone:2A9-1 Dil. Factor:20

CD80 Biolegend Cat.305230 Lot.b263714 Clone:2D10 Dil. Factor:20

CD11b Biolegend Cat.301322 Lot.B247020 Clone:ICRF44 Dil. Factor:20

CD8 BD Cat.555368 Lot.56785 Clone: RPA-T8 Dil. Factor: 5

CD3 Biolegend Cat.317331 Lot.b210179 Clone: OKT3 Dil. Factor: 20

CD38 Tonbo Cat.20-0389-T100 Lot.CD389061719203Clone: HIT2 Dil. Factor:20

HLA-DR BD Cat.552764 Lot.9080780 Clone: LN3 Dil. Factor: 20

CD25 Biolegend Cat.302624b Lot.b138300 Clone: BC96 Dil. Factor: 100

CD127 Invitrogen Cat.12-1278-42 Lot.2283989 Clone: RDR5 Dil. Factor:20

CD95 Biolegend Cat.305606 Lot.B107719 Clone: DX2 Dil. Factor:20

CTLA-4 Biolegend Cat.369616 Lot.b228178 Clone: BNI3 Dil. Factor:20

CD4 Tonbo Cat.65-0048 Lot.C004807121365C Clone: OKT4 Dil. Factor:100

CD134 Biolegend Cat.350012 Lot.B321854 Clone: BER-ACT35 Dil. Factor:20

CD69 Biolegend Cat.310804 Lot. b284767Clone: FN50 Dil. Factor:20

CD154 Biolegend Cat.310806 Lot.b317617 Clone: 24-31 Dil. Factor:20

CD137 Biolegend Cat.309810 Lot.b316126 Clone: 4B4-1 Dil. Factor:20

IL-6 BD Cat.551473 Lot.5292975 Clone: MQ2-6A3 Dil. Factor:5

TNFa BD Cat.502912b Lot.N/A Clone: MAb11 Dil. Factor:100

IFNa Miltenyi Biotech Cat.130-123-708 Lot.5210309856 Clone: LT27:295 Dil. Factor:50

Validation

All antibodies used are commercially available and have been validated by the manufacturers.

Biolegend: https://www.biolegend.com/en-us/quality/quality-control

Specificity testing of 1-3 target cell types with either single- or multi-color analysis (including positive and negative cell types).

Once specificity is confirmed, each new lot must perform with similar intensity to the in-date reference lot. Brightness (MFI) is

evaluated from both positive and negative populations.

Each lot product is validated by QC testing with a series of titration dilutions.

BD Biosciences: https://www.bdbiosciences.com/en-us/products/reagents/flow-cytometry-reagents/research-reagents

The BD Biosciences antibodies and reagents adhere to strict quality control (QC) SOPs and guidelines to ensure lot-to-lot consistency, which is critical for obtaining consistent and reproducible results. Our antibody specificities are confirmed for multiple applications, and all our flow cytometry reagents are titrated on the relevant positive and/or negative cells.

Tonbo: https://tonbobio.com/collections/flow-cytometry-reagents-1

Tonbo Biosciences flow cytometry reagents \& antibodies are manufactured with the highest quality and precision and validated for consistent performance in multiparametric flow cytometry experiments. Tonbo Biosciences offers a carefully selected portfolio of antibodies and fluorophores designed to provide researchers with a core resource for flow cytometry reagents that are used in the majority of staining protocols. Made in San Diego, CA, our manufacturing process includes optimization of fluorophore to protein ratio $(F: P)$, meticulous quality control testing, and stringent final release criteria, resulting in consistently high-performing reagents for multicolor protocols.

Beckman Coulter: https://www.beckman.com/reagents/coulter-flow-cytometry/antibodies-and-kits/single-color-antibodies Beckman Coulter offers the largest portfolio of CE-IVD and ASR conjugated antibodies validated against clinical standards. We develop and manufacture reagents according to current Good Manufacturing Practices (cGMP), the highest quality standards in the industry, ensuring optimal antibody panel performance.

Invitrogen: https://www.thermofisher.com/us/en/home/life-science/antibodies/antibody-performance-guarantee.html As a leading provider of high-quality, validated antibodies, our products are used by researchers around the world. Our customers purchase with confidence, knowing that we stand behind the quality of our antibodies with the Invitrogen ${ }^{\mathrm{TM}}$ antibody performance guarantee. If an Invitrogen antibody does not perform in your experiment as described on our website or data sheet, we will replace the product at no cost to you, or if you prefer, we will provide you with a credit for future purchase.

R\&D: https://www.rndsystems.com/products/rd-systems-approach-antibody-quality

Each antibody is manufactured under controlled conditions, undergoing rigorous quality control testing to ensure lot-to-lot

consistency and outstanding performance in all applications listed on our datasheets.

Miltenyi: https://www.miltenyibiotec.com/US-en/products/macs-antibodies/hybridomas/reafinity-recombinant-antibodies.html

\section{Human research participants}

\section{Policy information about studies involving human research participants}

Population characteristics

Samples were stratified by disease severity - healthy donors (HD), mild/moderate COVID-19, and severe COVID-19 and age ( $<60$ categorized as young and $\geq 60$ categorized as aged). Healthy Donors ( $n=49 ; 37$ young and 12 aged) included samples obtained from previous unrelated studies collected prior to 2018 and blood from seronegative healthcare workers collected after March 2020. Individuals with asymptomatic/mild disease ( $n=25 ; 13$ young and 12 aged) were identified as those that tested positive for SARS-CoV-2 during their visit to UCIMC for reasons unrelated to COVID-19 symptoms (e.g. heart attack, exacerbation of auto-immune disease, elective surgeries). These samples were obtained through the COVID-19 biospecimen 
bank at UCIMC. A total of 53 patients with severe COVID-19 (35 young and 16 aged) were profiled, including patients with severe illness requiring hospitalization (ward) and patients with critical illness requiring intensive care unit admission without/with intubation (ICU; ICU/I). For patients with severe disease, blood was collected longitudinally over several days post symptom (DPS) onset, with median DPS being 10 and 11 days for young and aged group respectively. Six patients (2 young and 4 aged) from our analysis succumbed to disease. Detailed characteristics of the cohorts and experimental breakdown by samples is provided in Supp Table 1.

Recruitment

Blood samples from patients admitted to University of California Irvine Medical Center (UCIMC) and participating in the NIH ACT-1 trial were used in these studies. Participants gave written consent to have the remainder of their blood samples used for ancillary studies related to COVID-19. Samples from healthy Donors were obtained from previous unrelated studies collected prior to 2018 and blood from seronegative healthcare workers collected after March 2020.

Ethics oversight

This study was approved by University of California Irvine Institutional Review Boards. Informed consent was obtained from all enrolled subjects.

Note that full information on the approval of the study protocol must also be provided in the manuscript.

\section{Flow Cytometry}

\section{Plots}

Confirm that:

\The axis labels state the marker and fluorochrome used (e.g. CD4-FITC).

W The axis scales are clearly visible. Include numbers along axes only for bottom left plot of group (a 'group' is an analysis of identical markers).

\All plots are contour plots with outliers or pseudocolor plots.

\A numerical value for number of cells or percentage (with statistics) is provided.

\section{Methodology}

Sample preparation

Instrument

Software

Cell population abundance

Gating strategy
Whole blood samples were collected in EDTA vacutainer tubes. Peripheral blood mononuclear cells (PBMC) and blood plasma samples were isolated after whole blood centrifugation $1200 \mathrm{~g}$ for 10 minutes at room temperature in SepMate tubes (STEMCELL Technologies). PBMC were cryo-preserved using 10\% DMSO/FBS and Mr. Frosty Freezing containers (Thermo Fisher Scientific) at -80C then transferred to a cryogenic unit until analysis. Frozen PBMCs were thawed, washed in FACS buffer (2\% FBS, 1mM EDTA in PBS) and counted on TC20 (Biorad) before surface staining using two independent flow panels.

Attune NxT acoustic focusing cytometer (Life Technologies).

Data were analyzed using FlowJo v10 (TreeStar, Ashland, OR, USA).

Cell sorting was for viable cells only, which were later counted with Trypan blue to note cell viability after the sort.

FSC-A/SSC-A was used to gate on live cells. FSC-A/FSC-H was used to gate on single cells. Dead cells positive for LIVE/DEAD stains were excluded.

\Tick this box to confirm that a figure exemplifying the gating strategy is provided in the Supplementary Information. 